\title{
Using Samples of Unequal Length in Generalized Method of Moments Estimation*
}

\author{
Anthony W. Lynch ${ }^{\dagger}$ \\ New York University and NBER \\ Jessica A. Wachter \\ University of Pennsylvania and NBER
}

October 3, 2004

\footnotetext{
*The authors would like to thank Robert Engle, Martin Lettau, Andrew Lo, Kenneth Singleton, Robert Stambaugh, Amir Yaron, and Motohiro Yogo as well as seminar participants at New York University, and at the Wharton School for their comments and suggestions. The most recent version of this paper can be found at http://finance.wharton.upenn.edu/ ${ }^{\sim}$ jwachter/research/gmm.pdf.

†'School of Business, New York University, 44 West Fourth Street, Suite 9-190, New York, NY 10012-1126, alynch@stern.nyu.edu, (212) 998-0350.

${ }_{\ddagger}^{\ddagger}$ Department of Finance, The Wharton School, University of Pennsylvania, 2300 SH-DH, Philadelphia, PA, 19104. jwachter@wharton.upenn.edu, (215) 898-7634.
} 


\title{
Using Samples of Unequal Length in Generalized Method of Moments Estimation
}

\begin{abstract}
Many applications in financial economics use data series with different starting or ending dates. This paper describes an estimation method, based on the generalized method of moments (GMM), which makes use of all available data for each moment condition. We introduce two asymptotically equivalent estimators that are consistent, asymptotically normal, and more efficient asymptotically than standard GMM. We illustrate these estimators in an application to mutual fund performance evaluation. Both estimators are extended to general patterns of missing data, and shown to be more efficient than estimators that ignore intervals of the data, and thus more efficient than standard GMM.
\end{abstract}




\section{Introduction}

Many applications in financial economics involve data series that have different starting dates, or, more rarely, different ending dates. Settings where some data series are available over a much shorter time frame than others include estimation and testing using international data, and performance evaluation of mutual funds. These problems represent only the most extreme examples of differences in data length. More broadly, aggregate stock return data may be available over a longer time frame than macroeconomic data, cash flow and earnings data, term structure data, or options data.

When data are missing as described above, common practice is to take the intersection of the sample periods over which the data are observed. The intersection then becomes the sample period for the study and the rest of the data are ignored. This paper introduces an alternative, based on the generalized method of moments (GMM), that allows the researcher to make use of all of the data available for each moment condition. ${ }^{1}$ We show, moreover, that our method is more efficient than standard GMM, and more efficient than introducing the data from the longer series in a "naive" way.

The problem of unequal sample lengths in financial time series was first addressed by Stambaugh (1997). ${ }^{2}$ Stambaugh derives a maximum likelihood and a Bayesian estimator for the mean and the variance of a joint normal distribution, assuming returns are homoscedastic and independently distributed, in a setting where some return series start at a later date than others. Little and Rubin (2002) also derive maximum likelihood estimators when a portion of the data is missing (generally in non-economic applications), but their approach is similarly dependent on the specifics of the data generating process. In contrast, our approach, because it is based on GMM, does not require the data generating process to be normal. It can be used for dependent, stationary processes, and it permits estimation of parameters that are related to the observed functions in non-linear ways. ${ }^{3}$ As shown in Cochrane (2001), many common estimation techniques used in finance can be seen as

\footnotetext{
${ }^{1}$ Burguete, Gallant, and Souza (1982) and Hansen (1982) describe the GMM estimator and derive its asymptotic properties. Hansen and Singleton (1982) derive implications for estimation and testing of financial models; Brandt (1999) derives implications for the estimation of optimal portfolio and consumption choice. See Newey and McFadden (1994) for a survey of recent work on GMM and related estimators.

${ }^{2}$ Pastor and Stambaugh $(2002 \mathrm{a}, 2002 \mathrm{~b})$ derive Bayesian posteriors for means and variances of mutual fund returns using samples of unequal length, under the assumption of normality and identically and independently distributed returns. Storesletten, Telmer, and Yaron (2004) combine a time series of macro-economic variables dating back to 1930 with the shorter Panel Study of Income Dynamics to estimate the relationship between cross-sectional variance and recessions.

${ }^{3}$ Another strand of literature considers the problem of $n$ independent individuals observed at up to $T$ time periods, where some individuals drop out of the study (see, e.g., Robins and Rotnitsky (1995)). The independence across individuals and the fact that asymptotics are derived as $n$, rather than $T$, approaches infinity differentiates this problem from the one considered here.
} 
special cases of GMM. Assumptions required for the consistency and asymptotic normality of the standard GMM estimator are also required here. In particular, we adopt the mixing assumption of White and Domowitz (1984) as a means of limiting the temporal dependence of the underlying stochastic process. ${ }^{4}$

Because our method is based on GMM, the results we derive are asymptotic. Developing an asymptotic approach to a problem of missing data may at first seem strange. After all, asymptotics involve taking the sample size to infinity, which seems in opposition to the notion of missing data. We argue, however, that it is no more strange than applying asymptotics in the usual setting, where samples have the same length. In such cases, the number of data points is, of course, finite, so the asymptotic distribution must be treated as an approximation to the true sampling distribution of the parameters. Our asymptotic distribution can be thought of as an approximation in exactly the same way.

So that this approximation is reasonable, care must be taken to insure that the missing data problem does not become trivial as the sample size becomes large. For this reason, we develop an asymptotic theory that keeps the fraction of missing data fixed as the sample size approaches infinity. To be precise, if $T$ denotes the length of the longer sample, we say that $\lambda T$ is the length of the shorter sample, for $0<\lambda \leq 1$. We hold $\lambda$ constant, as $T$ approaches infinity. This approach has a parallel in the simulated method of moments estimation technique (see Duffie and Singleton (1993)), where the length of the simulated series divided by the length of the observed series is assumed to be constant as the both series lengths approach infinity.

Our initial setting supposes that some moment conditions are observed over the full data set while others are observed over a data set that has the same ending date but a later starting date (we later generalize this to other patterns of missing data). The two sets of moment conditions may depend on the same or different underlying parameters. We develop two asymptotically equivalent estimators that make use of all of the data. While general, these estimators are straightforward to implement, as we show by illustrating our procedures in an estimation of mutual fund performance (Section 5), and have natural and intuitive interpretations.

The first estimator (which we call the adjusted-moment estimator) uses full sample averages to estimate the moments for which full-sample data are available, and short sample averages to estimate moments for which only short-sample data are available. Then the moments for which

\footnotetext{
${ }^{4}$ Under stationarity, mixing is a slightly stronger condition than ergodicity. Intuitively, mixing requires that autocovariances vanish as the lag length increases, but sufficiently slowly to allow processes with memories much longer than finite ARMA processes.
} 
only the short sample is available are "adjusted" using coefficients from a regression of the shortsample moments on the full-sample moments. This is reminiscent of an adjustment that appears in Stambaugh (1997) and Little and Rubin (2002) but here operates in a more general context. The second estimator, (which we call the over-identified estimator) uses the extra data available from the full sample as a new set of moment conditions. This estimator was suggested by Stambaugh (1997) and, in the linear context of that paper, turns out to be identical to our adjusted-moment estimator (and the maximum-likelihood estimator proposed in that paper). In the more general context of our paper, the two estimators are equivalent asymptotically but typically differ in finite samples. Singleton (2004) proposes an extension of the estimator in Stambaugh (1997) that is similar to our over-identified estimator, but does not formally show asymptotic normality or efficiency over standard GMM. We build on this work by showing that both of our estimators are consistent and asymptotically normal, and that both are asymptotically more efficient than standard GMM.

Our approach can be extended to many other patterns of missing data. One pattern of interest is the case where there are more than two starting dates but all series end at the same date (this case satisfies a condition that Little and Rubin (2002) call monotonicity). This pattern is analyzed in detail in a maximum likelihood setting for independent and identically distributed normal observations by Little and Rubin, and by Stambaugh (1997). Both of our estimators can be extended not only to this case, but further, to cases where the series do not satisfy monotonicity. The extension works for an arbitrary number of ending dates and starting dates. It is also possible to have data missing in the middle of the sample. Despite the general nature of this problem, it is still possible to prove that the adjusted-moment and the over-identified estimators are asymptotically equivalent, though different in finite samples. Each preserves key properties of its counterpart when there are only two starting dates. Moreover, we show that it is always more efficient to "add" an interval of data, even if some series are not observed over the interval. By implication, these generalized estimators are also more efficient than standard GMM.

The organization of the paper is as follows. The first section develops the asymptotic theory that will be the basis for the consistency and asymptotic normality proofs. The key result in this section is that sample averages (scaled appropriately) taken over disjoint intervals of data are independent as the number of data points in each interval approaches infinity. This result clearly holds when observations are independent. Even when there is dependence the result holds, provided that the process satisfies a mixing condition in the sense of White and Domowitz (1984). Intuitively, mixing insures that autocovariances are small at arbitrarily long lags. As the number of data points 
approaches infinity, the data from one partial sum that is "near" the data from the other partial sum becomes negligible in the overall average, implying that the partial sums are independent.

With this result as background, the second section defines four estimators in a setting where data are missing at the beginning of the sample for some of the moment conditions. The first of these estimators is the standard GMM estimator (we call it "short"). The second of these estimators combines the long and short data in a naive way (we call it "long"). The third and the fourth are the adjusted-moment and the over-identified estimator mentioned above. All four estimators are shown to be consistent and asymptotically normal under standard assumptions. Moreover, for each estimator, the efficient weighting matrix is the inverse of the variance-covariance matrix of the moments, just as in standard GMM.

The third section shows that the adjusted-moment estimator and the over-identified estimator are asymptotically equivalent. They are both more efficient than the short estimator (standard GMM), and more efficient than the long estimator (which takes into account the additional data in a naive way). The long estimator is not necessarily more efficient than the short estimator; thus including the additional data in a naive way could cause the efficiency of the estimators to deteriorate rather than improve. Fortunately, the adjusted-moment and the over-identified estimator are just as easy to compute as the short and long estimator. Finally, this section shows that in finite samples, the adjusted-moment and the over-identified estimators generally differ.

The fourth section investigates a special case in which the original system is exactly identified, and some variables can be identified by the long-sample data alone. In this case, it is possible to gain additional intuition about the forms of the adjusted-moment and over-identified estimators, and to estimate the size of the efficiency gain from using the adjusted-moment or the over-identified estimator. For simplicity, moments for which the full sample is available are assumed to depend on a subset of the parameters $\theta_{1}$, while moments for which only the short sample is available depend on $\theta_{2}$ as well as, possibly, $\theta_{1}$. Asymptotic standard errors for $\theta_{1}$ are a fraction $\sqrt{\lambda}$ of their values under standard GMM; thus the percent decrease is $1-\sqrt{\lambda}$. For $\theta_{2}, 1-\sqrt{\lambda}$ represents an upper bound on the percent decrease. The actual decrease depends on the correlation between the moment conditions, and the extent to which $\theta_{1}$ influences the moments for which only the short sample is available.

The fifth section illustrates our methods by estimating mutual fund performance relative to a conditional factor models. The mutual fund data is from Elton, Gruber, and Blake (1996), and begins in 1977. In contrast, data on factors and on information variables is available starting in 
1927. Using the stochastic discount factor formulation of factor models (e.g. Cochrane (2001)), we show that including the longer data allows for substantially more precise estimates of mutual fund performance than using the shorter data alone.

The sixth section extends the analysis to the general case, where there can be an arbitrary number of starting dates, ending dates, and data can be missing in the middle of the sample. Data interval endpoints are identified by points in time at which data for at least one sample moment starts or ends. Asymptotic theory is developed assuming that the ratios of these various data intervals remain constant as the sample size grows large. Both the adjusted-moment and overidentified estimators are extended in this more general setting in natural ways. The over-identified estimator is obtained by treating the sample moments for each data interval as separate sample moments in the GMM estimation. The adjusted-moment estimator is defined inductively: the moments used when a data interval is added are obtained by taking the moments used without that interval and adding an adjustment term that uses the data in the added interval. It is shown that these extensions to the two estimators, while different in finite samples, are asymptotically equivalent, and moreover, that adding an additional data interval, even though not observed for all moments, improves efficiency. The sixth section concludes.

\section{Large sample theory for sums covering different sample periods}

This section develops the asymptotic theory necessary for establishing properties of our estimators. The assumptions are standard (see, e.g. White and Domowitz (1984)). The principle new result is contained in Theorem 1.1: that partial sums taken over disjoint intervals are asymptotically independent and normally distributed.

Let $\left\{x_{t}\right\}_{t=-\infty}^{\infty}$ denote a $p$-component stochastic process defined over an underlying probability space $(\Omega, \mathcal{F}, P)$. Let $\mathcal{F}_{a}^{b} \equiv \sigma\left(x_{t} ; a \leq t \leq b\right)$, the Borel $\sigma$-algebra of events generated by $x_{a}, \ldots, x_{b}$. Consider a function $f: \mathbf{R}^{p} \times \Theta \rightarrow \mathbf{R}^{l}$ for $\Theta$, a compact subset of $\mathbf{R}^{q}$. The function $f$ provides the restrictions that determine $\theta$ based on the observations of $x_{t}$. In what follows we make standard assumptions on $\left\{x_{t}\right\}$ and $f$ in order to guarantee consistency and asymptotic normality of the estimates. Particularly useful is a notion of dependence known as mixing.

Following White and Domowitz (1984), define

$$
\alpha(\mathcal{F}, \mathcal{G}) \equiv \sup _{\{F \in \mathcal{F}, G \in \mathcal{G}\}}|P(F G)-P(F) P(G)|
$$


for $\sigma$-algebras $\mathcal{F}$ and $\mathcal{G}$, and

$$
\alpha(s) \equiv \sup _{t} \alpha\left(\mathcal{F}_{-\infty}^{t}, \mathcal{F}_{t+s}^{\infty}\right)
$$

The process $\left\{x_{t}\right\}$ is said to be $\alpha$-mixing if $\alpha(s) \rightarrow 0$ as $s \rightarrow \infty$. As White and Domowitz (1984) discuss, $\alpha$-mixing guarantees that autocovariances vanish at at arbitrarily long lags. Mixing is a convenient assumption because it allows a trade-off between the speed at which $\alpha(s)$ approaches zero and the conditions required on $\left\{x_{t}\right\}$. In particular, a process is said to be $\alpha$-mixing of size $r /(r-1)$ for $r>1$ if for some $\kappa>r /(r-1), \alpha(s)$ is $O\left(s^{-\kappa}\right)$. We assume that $\left\{x_{t}\right\}$ is mixing:

Assumption $1\left\{x_{t}\right\}_{t=-\infty}^{\infty}$ is $\alpha$-mixing of size $\frac{r}{r-1}$ for $r>1$, and stationary.

Assumption 2 guarantees that $f\left(x_{t}, \theta\right)$ is also mixing.

Assumption $2 f(\cdot, \theta)$ is measurable for all $\theta \in \Theta$.

The following assumption specifies the sense in which $f\left(x_{t}, \theta\right)$ determines $\theta$ given observations on $x_{t}$.

Assumption 3 There exists a unique $\theta_{0} \in \Theta$ such that $E\left[f\left(x_{t}, \theta_{0}\right)\right]=0$.

The next assumptions form the basis for the consistency and asymptotic normality results of estimators based on partial sums of $f\left(x_{t}, \theta\right)$.

Assumption 4 There exists $\Delta \in \mathbf{R}$ such that $E\left(\left|f_{i}\left(x_{t}, \theta_{0}\right)^{2 r}\right|\right)<\Delta, i=1, \ldots, l$.

Assumption $5 f\left(x_{t}, \theta\right)$ is continuous in $\theta$. There exists a measurable function $H\left(x_{t}\right) \in \mathbf{R}^{l}$ such that $\left|f_{i}\left(x_{t}, \theta\right)\right| \leq H_{i}\left(x_{t}\right)$ for all $\theta \in \Theta$ and such that $E\left|H_{i}\left(x_{t}\right)\right|^{r+\delta} \leq \Delta<\infty$, for some $\delta>0$ and all $i=1, \ldots, l$.

Assumptions 4 and 5 illustrate the usefulness of the definition of mixing. As White and Domowitz (1984) explain, the greater is $r$, the more dependence is allowed for the process $x_{t}$, but the stronger are the required conditions on the function $f$. For example, if $x_{t}$ is independent then $\alpha(s)=0$ for all $s$, and hence we can set $r=1$. If $x_{t}$ follows an ARMA process, $r$ can be taken to be arbitrarily close to 1 .

White and Domowitz (1984) prove the following:

Lemma 1.1 Assumptions 1 and 2 imply that $\left\{f\left(x_{t}, \theta\right)\right\}_{t=-\infty}^{\infty}$ is $\alpha-$ mixing of size $r /(r-1)$ and stationary. 
Following Hansen (1982), define the $l \times l$ matrix $R(\tau)=E\left[f\left(x_{0}, \theta_{0}\right) f\left(x_{-\tau}, \theta_{0}\right)^{\top}\right]$ and let

$$
S=\sum_{\tau=-\infty}^{\infty} R(\tau)=R(0)+\sum_{\tau=1}^{\infty}\left(R(\tau)+R(\tau)^{\top}\right) .
$$

Lemma 1.1 implies that this sum converges, because $\alpha$-mixing combined with stationarity implies that the series is ergodic (see White (1994, Proposition 3.44)). Define

$$
g_{T}(\theta)=\frac{1}{T} \sum_{t=1}^{T} f\left(x_{t}, \theta\right)
$$

for $\theta \in \Theta$, and

$$
w_{t}=f\left(x_{t}, \theta_{0}\right)
$$

The following lemma establishes that partial sums taken over disjoint intervals are asymptotically independent. This is stronger than showing that the partial sums of have zero covariance asymptotically.

Lemma 1.2 Let $F \in \mathcal{F}_{-\infty}^{0}$. Let $\mu$ be a $1 \times l$ vector, and let $c$ be a scalar. Then Assumptions 1-3 and 5 imply that

$$
\lim _{T \rightarrow \infty} P\left(\left(\sqrt{T} \mu g_{T}\left(\theta_{0}\right)<c\right) F\right)=\lim _{T \rightarrow \infty} P\left(\sqrt{T} \mu g_{T}\left(\theta_{0}\right)<c\right) P(F) .
$$

Proof For any integer $T$,

$$
\sqrt{T} g_{T}\left(\theta_{0}\right)=\frac{1}{\sqrt{T}} \sum_{t=1}^{\lfloor\sqrt{T}\rfloor} w_{t}+\frac{1}{\sqrt{T}} \sum_{t=\lfloor\sqrt{T}\rfloor+1}^{T} w_{t},
$$

where $\lfloor\sqrt{T}\rfloor$ is the largest integer less than the square root of $T$. Assumptions $1-3$, and 5 imply that

$$
\frac{1}{\sqrt{T}} \sum_{t=1}^{\lfloor\sqrt{T}\rfloor} w_{t}=\frac{\lfloor\sqrt{T}\rfloor}{\sqrt{T}} \frac{1}{\lfloor\sqrt{T}\rfloor} \sum_{t=1}^{\lfloor\sqrt{T}\rfloor} w_{t} \rightarrow \text { a.s. } 0
$$

as $T \rightarrow \infty$, by Theorem 2.3 of White and Domowitz (1984). Because

$$
\begin{gathered}
\frac{1}{\sqrt{T}} \sum_{t=\lfloor\sqrt{T}\rfloor+1}^{T} w_{t} \in \mathcal{F}_{\sqrt{T}}^{\infty}, \\
\left|P\left(\left[\frac{1}{\sqrt{T}} \sum_{t=\lfloor\sqrt{T}\rfloor+1}^{T} \mu w_{t}<c\right] F\right)-P\left(\frac{1}{\sqrt{T}} \sum_{t=\lfloor\sqrt{T}\rfloor+1}^{T} \mu w_{t}<c\right) P(F)\right|<\alpha(\sqrt{T}) .
\end{gathered}
$$




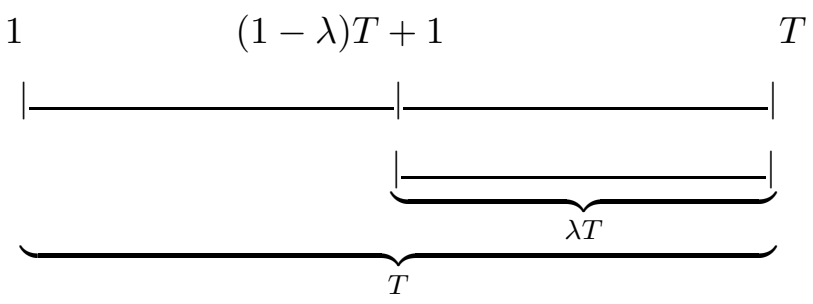

Figure 1: Notation for data missing at the start of the sample

By Lemma 1.1, $w_{t}$ is $\alpha$-mixing. Therefore $\alpha(\sqrt{T})$ goes to 0 as $T \rightarrow \infty$. By the Slutsky theorem,

$$
\begin{aligned}
\lim _{T \rightarrow \infty} P\left(\left(\sqrt{T} \mu g_{T}\left(\theta_{0}\right)<c\right) F\right) & =\lim _{T \rightarrow \infty} P\left(\left[\frac{1}{\sqrt{T}} \sum_{t=\lfloor\sqrt{T}\rfloor+1}^{T} \mu w_{t}<c\right] F\right) \\
& =\lim _{T \rightarrow \infty} P\left(\frac{1}{\sqrt{T}} \sum_{t=\lfloor\sqrt{T}\rfloor+1}^{T} \mu w_{t}<c\right) P(F) \\
& =\lim _{T \rightarrow \infty} P\left(\sqrt{T} \mu g_{T}\left(\theta_{0}\right)<c\right) P(F)
\end{aligned}
$$

where the second line follows from Lemma 1.1, and the last line follows from a repeated application of the Slutsky Theorem.

Let $\lambda$ be a rational number between 0 and 1 , and define $n_{0}$ to be the smallest positive integer $n$ such that $n \lambda$ is an integer. We consider partial sums of $f$ of length $\lambda T$ and $(1-\lambda) T$ for $T$ a multiple of $n_{0}$. For $T$ a multiple of $n_{0}$, define

$$
\begin{aligned}
g_{(1-\lambda) T}(\theta) & =\frac{1}{(1-\lambda) T} \sum_{t=1}^{(1-\lambda) T} f\left(x_{t}, \theta\right) \\
g_{\lambda T}(\theta) & =\frac{1}{\lambda T} \sum_{t=(1-\lambda) T+1}^{T} f\left(x_{t}, \theta\right) .
\end{aligned}
$$

Sums of $f$ are indexed by the length of the sample. This is a slight abuse of notation because the subscript $\lambda T$ does not refer to the sum taken over observations $1, \ldots, \lambda T$. Figure 1 illustrates the notation. The subscripts $\lambda T,(1-\lambda) T$ and $T$ can be understood as referring to intervals of the data rather than the ending point of the sample.

The following theorem is the main result of this section. For the remainder of the paper, we let $T$ approach infinity along the subsequence of integer multiples of $n_{0} \cdot{ }^{5}$

\footnotetext{
${ }^{5}$ Alternatively, we could define partial sums of length $\lambda n_{0} T^{\prime}$ and $(1-\lambda) n_{0} T^{\prime}$ for any integer $T^{\prime}$. The results would be identical, but the notation would be more cumbersome.
} 
Theorem 1.1 Assumptions 1-5 imply that as $T \rightarrow \infty$,

$$
\sqrt{T}\left(\begin{array}{l}
\sqrt{(1-\lambda)} g_{(1-\lambda) T}\left(\theta_{0}\right) \\
\sqrt{\lambda} g_{\lambda T}\left(\theta_{0}\right)
\end{array}\right) \rightarrow_{\mathrm{d}} N\left(0,\left[\begin{array}{ll}
S & 0 \\
0 & S
\end{array}\right]\right) .
$$

Proof Assumptions 1-4 imply that

$$
\sqrt{(1-\lambda) T} g_{(1-\lambda) T}\left(\theta_{0}\right) \rightarrow{ }_{\mathrm{d}} N(0, S)
$$

and

$$
\sqrt{\lambda T} g_{\lambda T}\left(\theta_{0}\right) \rightarrow_{\mathrm{d}} N(0, S)
$$

by Theorem 2.4 of White and Domowitz (1984). Stationarity of $x_{t}$ (Assumption 1) implies that random variables $f\left(x_{-(1-\lambda) T+1}, \theta\right), \ldots, f\left(x_{\lambda T}, \theta\right)$ have the same joint distribution as random variables $f\left(x_{1}, \theta\right), \ldots, f\left(x_{T}, \theta\right)$. Thus partial sums taken over $f\left(x_{-(1-\lambda) T+1}, \theta\right), \ldots, f\left(x_{\lambda T}, \theta\right)$ have the same distribution as the corresponding partial sums taken over $f\left(x_{1}, \theta\right), \ldots, f\left(x_{T}, \theta\right)$. Define

$$
\begin{aligned}
\tilde{g}_{\lambda T}(\theta) & =\frac{1}{\lambda T} \sum_{t=1}^{\lambda T} f\left(x_{t}, \theta\right) \\
\tilde{g}_{(1-\lambda) T}(\theta) & =\frac{1}{(1-\lambda) T} \sum_{t=0}^{(1-\lambda) T-1} f\left(x_{-t}, \theta\right) .
\end{aligned}
$$

It suffices to prove the results for $\tilde{g}_{\lambda T}$ and $\tilde{g}_{(1-\lambda) T}$.

Let $\mathcal{N}(c)$ denote the cumulative distribution function of the standard normal distribution evaluated at $c$. Let $\mu_{1}$ and $\mu_{2}$ be $1 \times l$ vectors such that $\mu_{1} \mu_{1}^{\top}=\mu_{2} \mu_{2}^{\top}=1$. By Lemma 1.2,

$$
\begin{aligned}
& \lim _{T \rightarrow \infty} P\left(\mu_{1} \sqrt{(1-\lambda) T} S^{-1} \tilde{g}_{(1-\lambda) T}\left(\theta_{0}\right)<c_{1}, \mu_{2} \sqrt{\lambda T} S^{-1} \tilde{g}_{\lambda T}\left(\theta_{0}\right)<c_{2}\right)= \\
& \quad \lim _{T \rightarrow \infty} P\left(\mu_{1} \sqrt{(1-\lambda) T} S^{-1} g_{(1-\lambda) T}\left(\theta_{0}\right)<c_{1}\right) \lim _{T \rightarrow \infty}\left(\mu_{2} \sqrt{\lambda T} S^{-1} \tilde{g}_{\lambda T}\left(\theta_{0}\right)<c_{2}\right)=\mathcal{N}\left(c_{1}\right) \mathcal{N}\left(c_{2}\right)
\end{aligned}
$$

for scalars $a$ and $b$. This shows $\tilde{g}_{\lambda T}\left(\theta_{0}\right)$ and $\tilde{g}_{(1-\lambda) T}\left(\theta_{0}\right)$ are asymptotically independent, and therefore that $g_{\lambda T}\left(\theta_{0}\right)$ and $g_{(1-\lambda) T}\left(\theta_{0}\right)$ are asymptotically independent. The result follows from (5) and (6).

\section{Consistency and asymptotic normality of estimators}

In many applications, it happens that data is missing for the early part of the sample period for some moment conditions (see Stambaugh (1997) for an application to international data and Section 5 of this paper for an application to mutual funds). In the notation of Section 1, some 
elements of the vector $x_{t}$ are observed for dates $1, \ldots, T$, while others are observed only for the last fraction $\lambda$ of the sample, namely dates $(1-\lambda) T+1, \ldots T$. In this section, we introduce estimators that are consistent and asymptotically normal in this setting.

Without loss of generality, partition the elements of $x_{t}$ so that $x_{t}=\left[x_{1 t}^{\top} x_{2 t}^{\top}\right]^{\top}$, where data on $x_{1 t} \in \mathbf{R}^{p_{1}}$ is assumed to be available for the full sample, and data on $x_{2 t} \in \mathbf{R}^{p_{2}}$ is assumed to be available for the last $\lambda T$ dates of the sample. Similarly, partition the elements of $f$ into those that depend only on $x_{1 t}$ and those that depend on both $x_{1 t}$ and $x_{2 t}: f\left(x_{t}, \theta\right)=\left[f_{1}\left(x_{1 t}, \theta\right)^{\top} f_{2}\left(x_{t}, \theta\right)^{\top}\right]^{\top}$, where $f_{1}: \mathbf{R}^{p_{1}} \times \Theta \rightarrow \mathbf{R}^{l_{1}}$, and $f_{2}: \mathbf{R}^{p} \times \Theta \rightarrow \mathbf{R}^{l_{2}}$. Analogously, let

$$
\begin{aligned}
g_{1 T}(\theta) & =\frac{1}{T} \sum_{t=1}^{T} f_{1}\left(x_{t}, \theta\right), \\
g_{1,(1-\lambda) T}(\theta) & =\frac{1}{(1-\lambda) T} \sum_{t=1}^{(1-\lambda) T} f_{1}\left(x_{t}, \theta\right), \\
g_{1, \lambda T}(\theta) & =\frac{1}{\lambda T} \sum_{t=(1-\lambda) T+1}^{T} f_{1}\left(x_{t}, \theta\right),
\end{aligned}
$$

and

$$
g_{2, \lambda T}(\theta)=\frac{1}{\lambda T} \sum_{t=(1-\lambda) T+1}^{T} f_{2}\left(x_{t}, \theta\right) .
$$

It is useful to define partitions of the matrix $S$ that correspond to the partitions of $f$ and $g$. Let $R_{i j}(\tau)$ be the $l_{i} \times l_{j}$ matrix

$$
R_{i j}(\tau)=E\left[f_{i}\left(x_{0}, \theta_{0}\right) f_{j}\left(x_{-\tau}, \theta_{0}\right)^{\top}\right], \quad i, j=1,2,
$$

and define

$$
S_{i j}=\sum_{\tau=-\infty}^{\infty} R_{i j}(\tau)
$$

Then

$$
S=\left[\begin{array}{ll}
S_{11} & S_{12} \\
S_{21} & S_{22}
\end{array}\right]
$$

It is also useful to define the matrix of coefficients from a regression of the second series on the first. This is the $l_{2} \times l_{1}$ matrix

$$
B_{21}=S_{21} S_{11}^{-1}
$$

The residual variance from this regression will be denoted $\Sigma$, where

$$
\Sigma=S_{22}-S_{21} S_{11}^{-1} S_{12}
$$


We consider four estimators, distinguished by their moment conditions. In what follows, we will emphasize the case where the weighting matrix converges almost surely to the inverse of the variance-covariance matrix of the moments. Define

$$
\begin{aligned}
h_{T}^{\mathcal{S}}(\theta) & =\left[\begin{array}{ll}
g_{1, \lambda T}(\theta)^{\top} & g_{2, \lambda T}(\theta)^{\top}
\end{array}\right]^{\top} \\
h_{T}^{\mathcal{L}}(\theta) & =\left[\begin{array}{ll}
g_{1, T}(\theta)^{\top} & g_{2, \lambda T}(\theta)^{\top}
\end{array}\right]^{\top} \\
h_{T}^{\mathcal{A}}(\theta) & =\left[g_{1, T}(\theta)^{\top}\left(g_{2, \lambda T}(\theta)+\hat{B}_{21, \lambda T}(1-\lambda)\left(g_{1,(1-\lambda) T}(\theta)-g_{1, \lambda T}(\theta)\right)\right)^{\top}\right]^{\top} \\
h_{T}^{\mathcal{I}}(\theta) & =\left[g_{1,(1-\lambda) T}(\theta)^{\top} g_{1, \lambda T}(\theta)^{\top} g_{2, \lambda T}(\theta)^{\top}\right]^{\top},
\end{aligned}
$$

where $\hat{B}_{21, \lambda T}$ is an $l_{2} \times l_{1}$ matrix such that $\hat{B}_{21, \lambda T} \rightarrow$ a.s. $B_{21}$. Let

$$
\hat{\theta}_{T}^{\mathcal{S}}=\operatorname{argmin}_{\theta} h_{T}^{\mathcal{S}}(\theta)^{\top} W_{T}^{\mathcal{S}} h_{T}^{\mathcal{S}}(\theta)
$$

The estimator $\hat{\theta}_{T}^{\mathcal{S}}$ corresponds to the standard GMM. Observations on $x_{1 t}$ for $t=1, \ldots,(1-\lambda) T$ are discarded. Let

$$
\hat{\theta}_{T}^{\mathcal{L}}=\operatorname{argmin}_{\theta} h_{T}^{\mathcal{L}}(\theta)^{\top} W_{T}^{\mathcal{L}} h_{T}^{\mathcal{L}}(\theta) .
$$

The estimator $\hat{\theta}_{T}^{\mathcal{L}}$ corresponds to incorporating all of the data in the most straightforward way. Let

$$
\hat{\theta}_{T}^{\mathcal{A}}=\operatorname{argmin}_{\theta} h_{T}^{\mathcal{A}}(\theta)^{\top} W_{T}^{\mathcal{A}} h_{T}^{\mathcal{A}}(\theta)
$$

and

$$
\hat{\theta}_{T}^{\mathcal{I}}=\operatorname{argmin}_{\theta} h_{T}^{\mathcal{I}}(\theta)^{\top} W_{T}^{\mathcal{I}} h_{T}^{\mathcal{I}}(\theta)
$$

Estimators $\hat{\theta}_{T}^{\mathcal{A}}$ and $\hat{\theta}_{T}^{\mathcal{I}}$ are less straightforward, but, as we will argue, superior ways of including the long data. Note that, because

$$
g_{1, T}=(1-\lambda) g_{1,(1-\lambda) T}+\lambda g_{1, \lambda T}
$$

the second component of (10) can be rewritten as

$$
h_{2, T}^{\mathcal{A}}=g_{2, \lambda T}+\hat{B}_{21, \lambda T}\left(g_{1, T}-g_{1, \lambda T}\right)
$$

Equation (16) illustrates the role of the longer sample in helping to estimate the second set of moment conditions. Consider for example the case where $g_{1}$ and $g_{2}$ are univariate. If $g_{1}$ is below average in the second part of the sample, and if $g_{1}$ and $g_{2}$ are positively correlated, $g_{2}$ is also likely to be below average. Thus the estimate of $E\left[f_{2}\left(x_{0}, \theta\right)\right]$ should be adjusted upward relative to $g_{2}$. We 
call $\hat{\theta}_{T}^{\mathcal{A}}$ the "adjusted moment" estimator, because it involves adjusting the second set of moments. We refer to $\hat{\theta}_{T}^{\mathcal{I}}$ as the "over-identified" estimator, because it involves adding an additional moment condition. As we will show, $\hat{\theta}_{T}^{\mathcal{I}}$ has the same asymptotic properties as $\hat{\theta}_{T}^{\mathcal{A}}$. In order to present theorems that apply to all the estimators, we use the notation $\hat{\theta}_{T}^{k}$ to denote a member of the class of estimators defined above, and similarly for $W^{k}$.

Theorem 2.1 Assumptions 1-5 imply that as $T \rightarrow \infty$,

$$
\sqrt{\lambda T} h_{T}^{k}\left(\theta_{0}\right) \rightarrow \mathrm{d} N\left(0, S^{k}\right)
$$

where

$$
\begin{aligned}
S^{\mathcal{S}} & =S \\
S^{\mathcal{L}} & =\left[\begin{array}{cc}
\lambda S_{11} & \lambda S_{12} \\
\lambda S_{21} & S_{22}
\end{array}\right] \\
S^{\mathcal{A}} & =\left[\begin{array}{cc}
S_{11}^{\mathcal{A}} & S_{12}^{\mathcal{A}} \\
S_{21}^{\mathcal{A}} & S_{22}^{\mathcal{A}}
\end{array}\right]=\left[\begin{array}{cc}
\lambda S_{11} & \lambda S_{12} \\
\lambda S_{21} & S_{22}-(1-\lambda) S_{21} S_{11}^{-1} S_{12}
\end{array}\right] \\
S^{\mathcal{I}} & =\left[\begin{array}{ccc}
\frac{\lambda}{1-\lambda} S_{11} & 0 & 0 \\
0 & S_{11} & S_{12} \\
0 & S_{21} & S_{22}
\end{array}\right] .
\end{aligned}
$$

Proof Equation (17) follows from Theorem 1.1. We show (19); the proofs of (18) and (20) are similar. In what follows, the argument $\theta_{0}$ is suppressed and convergence is in the sense of almost surely.

Stationarity implies that $S_{11}^{\mathcal{A}}=\lambda S_{11}$. By Theorem 1.1,

$$
\begin{aligned}
&\left.\lim _{T \rightarrow \infty} E\left[\sqrt{\lambda T}\left(\lambda g_{i, \lambda T}+(1-\lambda) g_{i,(1-\lambda) T}\right) \sqrt{\lambda T}\left(g_{j,(1-\lambda) T}-g_{j, \lambda T}\right)\right)^{\top}\right] \\
&=\lim _{T \rightarrow \infty}\left(-E\left[\sqrt{\lambda T} \lambda g_{i, \lambda T} \sqrt{\lambda T} g_{j, \lambda T}^{\top}\right]+E\left[\sqrt{\lambda T}(1-\lambda) g_{i,(1-\lambda T)} \sqrt{\lambda T} g_{j,(1-\lambda) T}^{\top}\right]\right) \\
&=\lambda S_{i j}-\lambda S_{i j}=0
\end{aligned}
$$

for $i, j=1,2$. Therefore,

$$
\begin{aligned}
S_{12}^{\mathcal{A}} & =\lim _{T \rightarrow \infty} E\left[\sqrt{\lambda T}\left(\lambda g_{1, \lambda T}+(1-\lambda) g_{1,(1-\lambda) T}\right) \sqrt{\lambda T}\left(g_{2, \lambda T}+B_{21}(1-\lambda)\left(g_{1,(1-\lambda) T}-g_{1, \lambda T}\right)\right)^{\top}\right] \\
& =\lim _{T \rightarrow \infty} E\left[\sqrt{\lambda T}\left(\lambda g_{1, \lambda T}+(1-\lambda) g_{1,(1-\lambda) T}\right) \sqrt{\lambda T} g_{2, \lambda T}^{\top}\right] \\
& =\lim _{T \rightarrow \infty} E\left[\sqrt{\lambda T} \lambda g_{1, \lambda T} \sqrt{\lambda T} g_{2, \lambda T}^{\top}\right] \\
& =\lambda S_{12} .
\end{aligned}
$$


The second line follows from (21) and the third and fourth lines follow from Theorem 1.1. Using similar reasoning,

$$
\begin{aligned}
S_{22}^{\mathcal{A}}= & \lim _{T \rightarrow \infty} E\left[\sqrt{\lambda T} g_{2, \lambda T} \sqrt{\lambda T} g_{2, \lambda T}^{\top}\right]-2 \lim _{T \rightarrow \infty}(1-\lambda) E\left[\sqrt{\lambda T} g_{2, \lambda T} \sqrt{\lambda T} g_{1, \lambda T}^{\top}\right] B_{21}^{\top} \\
& +\lim _{T \rightarrow \infty} B_{21}(1-\lambda)^{2} E\left[\sqrt{\lambda T}\left(g_{1,(1-\lambda) T}-g_{1, \lambda T}\right) \sqrt{\lambda T}\left(g_{1,(1-\lambda) T}-g_{1, \lambda T}\right)^{\top}\right] B_{21}^{\top} \\
= & S_{22}-2(1-\lambda) S_{21} S_{11}^{-1} S_{12}+(1-\lambda)^{2}\left(\frac{\lambda}{1-\lambda}+1\right) S_{21} S_{11}^{-1} S_{12} \\
= & S_{22}-(1-\lambda) S_{21} S_{11}^{-1} S_{12},
\end{aligned}
$$

which completes the derivation of (19).

To establish consistency, we require the following condition on the weighting matrices.

Assumption 6 For $k \in\{\mathcal{S}, \mathcal{L}, \mathcal{A}, \mathcal{I}\}$, the weighting matrix $W_{\lambda T}^{k}$ converges almost surely to a positive-definite matrix $W^{k}$.

Theorem 2.2 establishes consistency of the estimators.

Theorem 2.2 Assumptions 1-6 imply that as $T \rightarrow \infty, \hat{\theta}_{T}^{k} \rightarrow$ a.s. $\theta_{0}$ for $k \in\{\mathcal{S}, \mathcal{L}, \mathcal{A}, \mathcal{I}\}$.

Proof White and Domowitz (1984) show that under these assumptions

$$
\begin{gathered}
\left|g_{\lambda T}(\theta)-E f\left(x_{t}, \theta\right)\right| \rightarrow_{\text {a.s. }} 0 \\
\left|g_{(1-\lambda) T}(\theta)-E f\left(x_{t}, \theta\right)\right| \rightarrow_{\text {a.s. }} 0
\end{gathered}
$$

as $T \rightarrow \infty$ uniformly in $\theta \in \Theta$. By the continuous mapping theorem,

$$
h_{T}^{k}(\theta)^{\top} W_{T}^{k} h_{T}^{k}(\theta) \rightarrow \text { a.s. } E\left[f\left(x_{t}, \theta\right)\right]^{\top} W^{k} E\left[f\left(x_{t}, \theta\right)\right]
$$

for $k \in\{\mathcal{S}, \mathcal{L}, \mathcal{A}\}$, and

$$
h_{T}^{\mathcal{I}}(\theta)^{\top} W_{T}^{\mathcal{I}} h_{T}^{\mathcal{I}}(\theta) \rightarrow_{\text {a.s. }} E\left[f_{1}\left(x_{1 t}, \theta\right)^{\top} f\left(x_{t}, \theta\right)^{\top}\right]^{\top} W^{\mathcal{I}} E\left[\begin{array}{l}
f_{1}\left(x_{1 t}, \theta\right) \\
f\left(x_{t}, \theta\right)
\end{array}\right]
$$

uniformly in $\theta$. The result then follows from Amemiya (1985, Theorem 4.1.1).

Three remaining assumptions allow us to establish asymptotic normality of the estimators:

Assumption $7 \theta_{0}$ lies in the interior of $\Theta$.

Assumption $8 f(x, \theta)$ is continuously differentiable in $\theta$. 
Assumption 9 There exists a measurable matrix-valued function $\hat{H}\left(x_{t}\right) \in \mathbf{R}^{l \times q}$ such that $\left|\frac{\partial f_{i}}{\partial \theta_{j}}\left(x_{t}, \theta\right)\right|<$ $\hat{H}\left(x_{t}\right)_{(i, j)}$ for all $\theta$ in the interior of $\Theta$ and such that for some $\delta>0, E\left|\hat{H}\left(x_{t}\right)_{(i, j)}\right|^{r+\delta} \leq \Delta<\infty$ for all $i=1, \ldots, l, j=1, \ldots q$.

Define $D_{0, i}=E\left[\left.\left(\partial f_{i} / \partial \theta\right)\right|_{\theta_{0}}\right]$ and $D_{0}=\left[\begin{array}{ll}D_{0,1}^{\top}, & D_{0,2}^{\top}\end{array}\right]^{\top}$. Let

$$
\begin{aligned}
& D_{0}^{k}=D_{0} \quad k \in\{\mathcal{S}, \mathcal{L}, \mathcal{A}\} \\
& D_{0}^{\mathcal{I}}=\left[\begin{array}{lll}
D_{0,1}^{\top} & D_{0,1}^{\top} & D_{0,2}^{\top}
\end{array}\right]^{\top} .
\end{aligned}
$$

The following theorem establishes asymptotic normality.

Theorem 2.3 Assumptions 1-9 imply

$$
\sqrt{\lambda T}\left(\hat{\theta}_{T}^{k}-\theta_{0}\right) \rightarrow_{\mathrm{d}} N\left(0,\left(\left(D_{0}^{k}\right)^{\top} W^{k} D_{0}^{k}\right)^{-1}\left(\left(D_{0}^{k}\right)^{\top} W^{k} S^{k} W^{k} D_{0}^{k}\right)\left(\left(D_{0}^{k}\right)^{\top} W^{k} D_{0}^{k}\right)^{-1}\right) .
$$

Proof Define

$$
D_{T}^{k}(\theta)=\frac{\partial h_{T}^{k}}{\partial \theta}(\theta)
$$

for $\theta$ in the interior of $\Theta$. For $T$ sufficiently large, $\hat{\theta}_{T}^{k}$ lies in the interior of $\Theta$. By the mean value theorem, there exists a $\tilde{\theta}^{k}$ in the segment between $\theta_{0}$ and $\hat{\theta}_{T}^{k}$ such that

$$
h_{T}^{k}\left(\hat{\theta}_{T}^{k}\right)-h_{T}^{k}\left(\theta_{0}\right)=D_{T}^{k}\left(\tilde{\theta}^{k}\right)\left(\hat{\theta}_{T}^{k}-\theta_{0}\right) .
$$

Pre-multiplying by $D_{T}^{k}\left(\hat{\theta}_{T}^{k}\right)^{\top} W_{T}^{k}$ :

$$
D_{T}^{k}\left(\hat{\theta}_{T}^{k}\right)^{\top} W_{T}^{k}\left(h_{T}^{k}\left(\hat{\theta}_{T}^{k}\right)-h_{T}^{k}\left(\theta_{0}\right)\right)=D_{T}^{k}\left(\hat{\theta}_{T}^{k}\right)^{\top} W_{T}^{k} D_{T}^{k}\left(\tilde{\theta}^{k}\right)\left(\hat{\theta}_{T}^{k}-\theta_{0}\right) .
$$

By the first-order condition of the optimization problem,

$$
D_{T}^{k}\left(\hat{\theta}_{T}^{k}\right)^{\top} W_{T}^{k} D_{T}^{k}\left(\tilde{\theta}^{k}\right)\left(\hat{\theta}_{T}^{k}-\theta_{0}\right)=-D_{T}^{k}\left(\hat{\theta}_{T}^{k}\right)^{\top} W_{T}^{k} h_{T}^{k}\left(\theta_{0}\right) .
$$

The assumptions and Theorem 2.3 of White and Domowitz (1984) imply that

$$
D_{T}^{k}(\theta) \rightarrow \text { a.s. } E\left[\frac{\partial f}{\partial \theta}\left(x_{t}, \theta\right)\right]
$$

for $k \in\{\mathcal{S}, \mathcal{L}, \mathcal{A}\}$, and

$$
D_{T}^{\mathcal{I}}(\theta) \rightarrow_{\text {a.s. }} E\left[\begin{array}{l}
\frac{\partial f_{1}}{\partial \theta}\left(x_{1 t}, \theta\right) \\
\frac{\partial f}{\partial \theta}\left(x_{t}, \theta\right)
\end{array}\right]
$$


uniformly in $\theta$. Therefore by Theorem 2.2 and Assumptions 7 and 8, Amemiya (1985, Theorem 4.1.5) implies

$$
\begin{gathered}
D_{T}^{k}\left(\hat{\theta}_{T}^{k}\right) \rightarrow \text { a.s. } D_{0}^{k} \\
D_{T}^{k}\left(\tilde{\theta}^{k}\right) \rightarrow \text { a.s. } D_{0}^{k} \\
W_{T}^{k} \rightarrow_{\text {a.s. }} W^{k} .
\end{gathered}
$$

The result follows from the Slutsky Theorem.

As in Hansen (1982) choosing the weighting matrix that is a consistent estimator of the inverse variance-covariance matrix is efficient for a given set of moment conditions.

Theorem 2.4 Suppose $W_{\lambda T}^{k} \rightarrow$ a.s. $W_{k}=\left(S^{k}\right)^{-1}$. Then Assumptions $1-5$ and $7-9$

$$
\sqrt{\lambda T}\left(\hat{\theta}_{T}^{k}-\theta_{0}\right) \rightarrow_{\mathrm{d}} N\left(0,\left(\left(D_{0}^{k}\right)^{\top}\left(S^{k}\right)^{-1}\left(D_{0}^{k}\right)\right)^{-1}\right) .
$$

Moreover, this choice of $W^{k}$ is efficient for each estimator.

\section{Comparison}

The previous section defined four estimators: the "short" estimator, which corresponds to standard GMM; the "long" estimator, which makes use of the full sample in a naive way; and the adjusted-moment and over-identified estimators, which make use of the full sample in a slightly more complicated way than "long". These four estimators were shown to be consistent and asymptotically normal. This section compares the asymptotic efficiency of these estimators.

The first result is that the over-identified estimator and the adjusted-moment estimator have identical asymptotic properties when the optional weighting matrix is used.

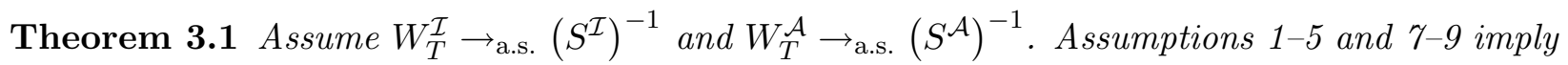
that the asymptotic distribution of $\sqrt{\lambda T} \hat{\theta}_{T}^{\mathcal{I}}$ is identical to that of $\sqrt{\lambda T} \hat{\theta}_{T}^{\mathcal{A}}$.

Proof It suffices to compare the asymptotic variances as the mean of both asymptotic distributions is $\sqrt{\lambda T} \theta_{0}$. In the case of the over-identified estimator, the inverse of the asymptotic variance of $\sqrt{\lambda T} \hat{\theta}_{T}^{\mathcal{I}}$ equals

$$
\begin{aligned}
\left(D^{\mathcal{I}}\right)^{\top}\left(S^{\mathcal{I}}\right)^{-1} D^{\mathcal{I}} & =\frac{1-\lambda}{\lambda} D_{0,1}^{\top} S_{11}^{-1} D_{0,1}+D_{0,1}^{\top} S_{11}^{-1} D_{0,1}+D_{0}^{\top}\left[\begin{array}{cc}
B_{21}^{\top} \Sigma^{-1} B_{21} & -B_{21}^{\top} \Sigma^{-1} \\
-\Sigma^{-1} B_{21} & \Sigma^{-1}
\end{array}\right] D_{0} \\
& =\frac{1}{\lambda} D_{0,1}^{\top} S_{11}^{-1} D_{0,1}+D_{0}^{\top}\left[\begin{array}{cc}
B_{21}^{\top} \Sigma^{-1} B_{21} & -B_{21}^{\top} \Sigma^{-1} \\
-\Sigma^{-1} B_{21} & \Sigma^{-1}
\end{array}\right] D_{0},
\end{aligned}
$$


where $\Sigma$ is defined by (7). This follows from Theorem 2.4 and Lemma A.3.

It follows from the distribution of the adjusted moment estimator (27) and Lemma A.3 that the inverse of the variance of $\sqrt{\lambda T} \hat{\theta}_{T}^{\mathcal{A}}$ equals

$$
D_{0}^{\top}\left(S^{\mathcal{A}}\right)^{-1} D_{0}=\frac{1}{\lambda} D_{0,1}^{\top} S_{11}^{-1} D_{0,1}+D_{0}^{\top}\left[\begin{array}{cc}
B_{21}^{\top} \Sigma^{-1} B_{21} & -B_{21}^{\top} \Sigma^{-1} \\
-\Sigma^{-1} B_{21} & \Sigma^{-1}
\end{array}\right] D_{0}
$$

which equals (28). Thus the estimators are asymptotically equivalent.

Theorem 3.1 shows that asymptotically, the distributions of the two estimators are the same. However, the interpretation of the over-identified estimator is different from the adjusted-moment estimator. Rather than adjusting the second set of moments based on the covariance with the first, the over-identified estimator turns the early data into a new moment condition.

Now we ask whether there is indeed an efficiency gain from using the longer sample. Are the adjusted-moment estimator and the over-identified estimator indeed more efficient than the short estimator?

Theorem 3.2 Assume 1-5 and 7-9 then

(1) If $W_{\lambda T}^{k} \rightarrow$ a.s. $\left(S^{k}\right)^{-1}$, for $k \in \mathcal{S}, \mathcal{A}, \mathcal{I}$, the estimators $\hat{\theta}_{T}^{\mathcal{A}}$ and $\hat{\theta}_{T}^{\mathcal{I}}$ are asymptotically more efficient than $\hat{\theta}_{T}^{S}$.

(2) If $W_{\lambda T}^{k} \rightarrow$ a.s. $W^{k}$ for $W^{k}$ positive definite, and such that $W^{\mathcal{A}}=W^{\mathcal{S}}$ almost surely, $\hat{\theta}_{T}^{\mathcal{A}}$ is more efficient than $\hat{\theta}_{T}^{\mathcal{S}}$.

Proof We first prove statement (1) for $\hat{\theta}_{T}^{\mathcal{A}}$. By Theorem 2.4 and Lemma A.2, it suffices to show that $S-S^{\mathcal{A}}$ is positive semi-definite. Note

$$
S-S^{\mathcal{A}}=(1-\lambda)\left[\begin{array}{cc}
S_{11} & S_{12} \\
S_{21} & S_{21} S_{11}^{-1} S_{12}
\end{array}\right] .
$$

For any $l \times 1$ vector $v=\left[v_{1}^{\top}, v_{2}^{\top}\right]^{\top}$,

$$
\begin{aligned}
v^{\top}\left(S-S^{\mathcal{A}}\right) v & =(1-\lambda)\left(v_{1}^{\top} S_{11} v_{1}+v_{1}^{\top} S_{12} v_{2}+v_{2}^{\top} S_{21} v_{1}+v_{2}^{\top} S_{21} S_{11}^{-1} S_{12} v_{2}\right) \\
& =(1-\lambda)\left(v_{1}^{\top} S_{11}^{\top} S_{11}^{-1} S_{11} v_{1}+v_{1}^{\top} S_{11} S_{11}^{-1} S_{12} v_{2}+v_{2}^{\top} S_{12}^{\top} S_{11}^{-1} S_{11} v_{1}+v_{2} S_{12}^{\top} S_{11}^{-1} S_{12} v_{2}\right) \\
& =(1-\lambda)\left(S_{11} v_{1}+S_{12} v_{2}\right)^{\top} S_{11}^{-1}\left(S_{11} v_{1}+S_{12} v_{2}\right) \geq 0
\end{aligned}
$$

because $S_{11}^{-1}$ is positive-semi-definite and $\lambda<1$. Therefore $S-S^{\mathcal{A}}$ is positive semi-definite and, as a consequence, $\hat{\theta}_{T}^{\mathcal{A}}$ is more efficient than $\hat{\theta}_{T}^{\mathcal{S}}$. The statement for $\hat{\theta}_{T}^{\mathcal{I}}$ then follows from Theorem 3.1. 
To prove statement (2), define

$$
U=W^{\mathcal{A}} D_{0}^{\mathcal{A}}\left(\left(D_{0}^{\mathcal{A}}\right)^{\top} W^{\mathcal{A}} D_{0}^{\mathcal{A}}\right)^{-1}
$$

Because the weighting matrix is assumed to be the same for both estimators,

$$
U=W^{\mathcal{S}} D_{0}^{\mathcal{S}}\left(\left(D_{0}^{\mathcal{S}}\right)^{\top} W^{\mathcal{S}} D_{0}^{\mathcal{S}}\right)^{-1}
$$

By Theorem 2.3, proving (2) is equivalent to showing $U^{\top} S U-U^{\top} S^{\mathcal{A}} U$ is positive semi-definite. But for any vector $\mathrm{v}$,

$$
v^{\top}\left(U^{\top} S U-U^{\top} S^{\mathcal{A}} U\right) v=(U v)^{\top}\left(S-S^{\mathcal{A}}\right) U v>0
$$

because $S-S^{\mathcal{A}}$ is positive semi-definite. Therefore $\hat{\theta}_{T}^{\mathcal{A}}$ is more efficient then $\hat{\theta}_{T}^{\mathcal{S}}$ when $W^{\mathcal{S}}=W^{\mathcal{A}}$.

Note that statement (2) of Theorem 3.2 does not make sense for the over-identified estimator $\hat{\theta}_{T}^{\mathcal{I}}$ because $\hat{\theta}_{T}^{\mathcal{I}}$ has $l_{1}$ more moment conditions than $\hat{\theta}_{T}^{\mathcal{A}}$ and $\hat{\theta}_{T}^{\mathcal{S}}$. It is not possible to keep the weighting matrices the same.

Theorem 3.2 shows that introducing the extra data from the longer series reduces the variance of the estimates relative to using the shorter series alone. It is also interesting to ask whether the estimator is more efficient than the one that would result from using the longer sample in a more "naive" way, namely using the longer data series to estimate the first set of moment conditions, and the shorter series to estimate the second. In the terminology of Section 1 this involves comparing $\hat{\theta}_{T}^{\mathcal{A}}$ with the estimator $\hat{\theta}_{T}^{\mathcal{L}}$.

Theorem 3.3 Assume 1-5 and 7-9 then

(1) If $W_{\lambda T}^{k} \rightarrow$ a.s. $\left(S^{k}\right)^{-1}$, for $k \in \mathcal{L}, \mathcal{A}, \mathcal{I}$, the estimators $\hat{\theta}_{T}^{\mathcal{A}}$ and $\hat{\theta}_{T}^{\mathcal{I}}$ are asymptotically more efficient than $\hat{\theta}_{T}^{\mathcal{L}}$.

(2) If $W_{\lambda T}^{k} \rightarrow$ a.s. $W^{k}$ for $W^{k}$ positive definite, and such that $W^{\mathcal{A}}=W^{\mathcal{L}}$ almost surely, $\hat{\theta}_{T}^{\mathcal{A}}$ is more efficient than $\hat{\theta}_{T}^{\mathcal{L}}$.

Proof As in Theorem 3.2, it suffices to show that

$$
S^{\mathcal{L}}-S^{\mathcal{A}}=\left[\begin{array}{cc}
0 & 0 \\
0 & (1-\lambda) S_{21} S_{11}^{-1} S_{12}
\end{array}\right]
$$


is positive semi-definite. Note that for any vector $v=\left[v_{1}^{\top}, v_{2}^{\top}\right]^{\top}$,

$$
v^{\top}\left(S^{\mathcal{L}}-S^{\mathcal{A}}\right) v=(1-\lambda)\left(S_{12} v_{2}\right)^{\top} S_{11}^{-1} S_{12} v_{2} \geq 0
$$

because $\lambda<1$ and $S_{11}$ is positive semi-definite. Lemma A.2 then implies that $\hat{\theta}_{T}^{\mathcal{A}}$ is more efficient than $\hat{\theta}_{T}^{\mathcal{L}}$. By Theorem 3.1, $\hat{\theta}_{T}^{\mathcal{I}}$ is also more efficient then $\hat{\theta}_{T}^{\mathcal{L}}$. This proves (1).

To show the second statement, define $U$ analogously to (29):

$$
U=W^{\mathcal{L}} D_{0}^{\mathcal{L}}\left(\left(D_{0}^{\mathcal{L}}\right)^{\top} W^{\mathcal{L}} D_{0}^{\mathcal{L}}\right)^{-1}
$$

and note that $W^{\mathcal{A}}=W^{\mathcal{L}}$. Because $S^{\mathcal{L}}-S^{\mathcal{A}}$ is positive semi-definite, for any vector $c$,

$$
v^{\top}\left(U^{\top} S^{\mathcal{L}} U-U^{\top} S^{\mathcal{A}} U\right) v=(U v)^{\top}\left(S^{\mathcal{L}}-S^{\mathcal{A}}\right) U v>0
$$

By Theorem 2.3, $\hat{\theta}_{T}^{\mathcal{A}}$ is more efficient then $\hat{\theta}_{T}^{\mathcal{L}}$ when $W^{\mathcal{A}}=W^{\mathcal{L}}$. This proves $(2)$.

Surprisingly, $\hat{\theta}_{T}^{\mathcal{L}}$ is not necessarily more efficient than $\hat{\theta}_{T}^{\mathcal{S}}$. Efficiency would require that

$$
S^{\mathcal{L}}-S=(1-\lambda)\left[\begin{array}{cc}
S_{11} & S_{12} \\
S_{21} & 0
\end{array}\right]
$$

be positive semi-definite. However, if the covariances between the first and second set of moment conditions are nonzero, this may not be the case. Thus it is not sufficient to simply use the first part of the sample, it must be combined with the second part of the sample in precisely the right way to produce a gain in efficiency.

We further explore the relation between these estimators by looking at the first order conditions. For the purpose of this discussion, we assume $W_{T}^{\mathcal{I}}=\left(S^{\mathcal{I}}\right)^{-1}, W_{T}^{\mathcal{A}}=\left(S^{\mathcal{A}}\right)^{-1}$, and $\hat{B}_{21, \lambda T}=B_{21}$. Differentiating (15) with respect to $\theta$ yields

$$
\begin{aligned}
0=\frac{1-\lambda}{\lambda} g_{1,(1-\lambda) T}^{\top} S_{11}^{-1} \frac{\partial g_{1,(1-\lambda) T}}{\partial \theta}+ & g_{1, \lambda T}^{\top} S_{11}^{-1} \frac{\partial g_{1, \lambda T}}{\partial \theta} \\
& +\left[\begin{array}{ll}
g_{1, \lambda T}^{\top} & g_{2, \lambda T}^{\top}
\end{array}\right]\left[\begin{array}{cc}
B_{21}^{\top} \Sigma^{-1} B_{21} & -B_{21}^{\top} \Sigma^{-1} \\
-\Sigma^{-1} B_{21} & \Sigma^{-1}
\end{array}\right]\left(\begin{array}{c}
\frac{\partial g_{1, \lambda T}}{\partial \theta} \\
\frac{\partial g_{2, \lambda T}}{\partial \theta}
\end{array}\right) \\
=\frac{1-\lambda}{\lambda} g_{1,(1-\lambda) T}^{\top} S_{11}^{-1} \frac{\partial g_{1,(1-\lambda) T}}{\partial \theta}+ & g_{1, \lambda T}^{\top} S_{11}^{-1} \frac{\partial g_{1, \lambda T}}{\partial \theta} \\
& +\left(g_{2, \lambda T}-B_{21} g_{1, \lambda T}\right)^{\top} \Sigma^{-1} \frac{\partial}{\partial \theta}\left(g_{2, \lambda T}-B_{21} g_{1, \lambda T}\right) .
\end{aligned}
$$

Equation (30) is the first-order condition that determines the over-identified estimator $\hat{\theta}_{T}^{\mathcal{I}}$. By contrast, the first order condition associated with (14) is

$$
\frac{1}{\lambda} g_{1, T}^{\top} S_{11}^{-1} \frac{\partial g_{1, T}}{\partial \theta}+\left[g_{1, T}^{\top} h_{2, T}^{\top}\right]\left[\begin{array}{cc}
B_{21}^{\top} \Sigma^{-1} B_{21} & -B_{21}^{\top} \Sigma^{-1} \\
-\Sigma^{-1} B_{21} & \Sigma^{-1}
\end{array}\right]\left(\begin{array}{c}
\frac{\partial g_{1, T}}{\partial \theta} \\
\frac{\partial h_{2, T}}{\partial \theta}
\end{array}\right)=0,
$$


which reduces to

$$
\begin{aligned}
0 & =\frac{1}{\lambda} g_{1, T}^{\top} S_{11}^{-1} \frac{\partial g_{1, T}}{\partial \theta}+\left(B_{21} g_{1, T}-h_{2, T}\right)^{\top} \Sigma^{-1} \frac{\partial}{\partial \theta}\left(B_{21} g_{1, T}-h_{2, T}\right) \\
& =\frac{1}{\lambda} g_{1, T}^{\top} S_{11}^{-1} \frac{\partial g_{1, T}}{\partial \theta}+\left(B_{21} g_{1, \lambda T}-g_{2, \lambda T}\right)^{\top} \Sigma^{-1} \frac{\partial}{\partial \theta}\left(B_{21} g_{1, \lambda T}-g_{2, \lambda T}\right) .
\end{aligned}
$$

Equation (31) is the first-order condition that determines the adjusted-moment estimator $\hat{\theta}_{T}^{\mathcal{A}}$. According to Theorem 3.1, these two first order conditions must be equivalent as $T \rightarrow \infty$. Indeed they are, because

$$
\left.\lim _{T \rightarrow \infty} \frac{\partial g_{1,(1-\lambda) T}}{\partial \theta}\right|_{\hat{\theta}_{T}^{\mathcal{I}}}=\left.\lim _{T \rightarrow \infty} \frac{\partial g_{1, \lambda T}}{\partial \theta}\right|_{\hat{\theta}_{T}^{I}}=\left.\lim _{T \rightarrow \infty} \frac{\partial g_{1, T}}{\partial \theta}\right|_{\hat{\theta}_{T}^{A}}=D_{0,1}
$$

and

$$
\begin{aligned}
\frac{1-\lambda}{\lambda} g_{1,(1-\lambda) T}^{\top} S_{11}^{-1} D_{0,1}+g_{1, \lambda T}^{\top} S_{11}^{-1} D_{0,1} & =\frac{1}{\lambda}\left((1-\lambda) g_{1,(1-\lambda) T}^{\top}+\lambda g_{1, \lambda T}^{\top}\right) S_{11}^{-1} D_{0,1} \\
& =\frac{1}{\lambda} g_{1, T}^{\top} S_{11}^{-1} D_{0} .
\end{aligned}
$$

In finite samples however, they will generally be equivalent only when

$$
\frac{\partial g_{1,(1-\lambda) T}}{\partial \theta}=\frac{\partial g_{1, \lambda T}}{\partial \theta}
$$

which occurs, for example, when the moment conditions are linear. This corresponds to the case examined by Stambaugh (1997) in a maximum likelihood context.

\section{A special case}

This section examines a special case of the set-up of Section 2. We assume the system is exactly identified, and that the variables can be decomposed into $\theta=\left[\begin{array}{ll}\theta_{1}^{\top} & \theta_{2}^{\top}\end{array}\right]^{\top}$, where $f_{1}$ is a function of $\theta_{1}$ alone. In this setting, we can draw additional conclusions about the first-order conditions of the adjusted-moment and over-identified estimators, and we can quantify the gains from including the longer sample.

Let $l_{1}$ be the length of $\theta_{1}$, and $l_{2}=q-l_{1}$ the length of $\theta_{2}$. For convenience assume that $W_{T}^{\mathcal{I}}=\left(S^{\mathcal{I}}\right)^{-1}$ and $\hat{B}_{21, T}=B_{21}$. Because

$$
\frac{\partial g_{1,(1-\lambda) T}}{\partial \theta_{2}} \equiv \frac{\partial g_{1, \lambda T}}{\partial \theta_{2}} \equiv \frac{\partial g_{1 T}}{\partial \theta_{2}} \equiv 0
$$

and because $\Sigma^{-1} \frac{\partial}{\partial \theta}\left(B_{21} g_{1, \lambda T}-g_{2, \lambda T}\right)$ is invertible, the first order conditions for the over-identified estimator $\hat{\theta}_{T}^{\mathcal{I}}$ reduce to

$$
g_{2, \lambda T}-B_{21} g_{1, \lambda T}=0
$$


for $\theta_{2}$, and thus

$$
\frac{1-\lambda}{\lambda} g_{1,(1-\lambda) T}^{\top} S_{11}^{-1} \frac{\partial g_{1,(1-\lambda) T}}{\partial \theta_{1}}+g_{1, \lambda T}^{\top} S_{11}^{-1} \frac{\partial g_{1, \lambda T}}{\partial \theta_{1}}=0
$$

for $\theta_{1}$. In comparison, the first order conditions for the adjusted-moment estimator $\hat{\theta}_{T}^{\mathcal{A}}$ reduce to (32) for $\theta_{2}$ but to $g_{1, T}=0$ for $\theta_{1}$. This is no surprise. When the adjusted-moment estimator is exactly identified, the first-order conditions must be equivalent to setting $g_{1, T}$ equal to zero, and $h_{2, T}$ equal to zero. When $g_{1, T}=0, h_{2, T}$ is equivalent to the left-hand side of (32).

We have shown that in the case considered here, the adjusted-moment estimator gives the same estimate for $\theta_{1}$ as simply using the full sample. The over-identified estimator gives a possibly different estimate, one that depends on the point in time in which the second series begins. While this dependence is possibly unattractive, (33) nonetheless has an interpretation: it is a weighted average of the moment conditions from the first part and the second part of the sample, where the weights are proportional to the derivatives, and thus to the amount of information contained in each part of the sample.

We now quantify the effects of using the adjusted-moment estimator or the over-identified estimator on the standard errors for $\theta$. In the special case where the system is exactly identified and $f_{1}$ depends on $\theta_{1}$, the derivative matrix $D_{0}$ is invertible and takes the form

$$
D_{0}=\left(\begin{array}{c}
D_{0,1} \\
D_{0,2}
\end{array}\right)=\left[\begin{array}{cc}
d_{11} & 0 \\
d_{21} & d_{22}
\end{array}\right]
$$

for an $l_{1} \times l_{1}$ invertible matrix $d_{11}$, an $l_{2} \times l_{1}$ matrix $d_{21}$ and an $l_{2} \times l_{2}$ invertible matrix $d_{22}$. The matrix $d_{11}$ gives the derivatives of $f_{1}$ with respect to $\theta_{1}, d_{21}$ gives the derivatives of $f_{2}$ with respect to $\theta_{1}$, and $d_{22}$ gives the derivatives of $f_{2}$ with respect to $\theta_{2}$.

The inverse of $D_{0}$ takes the form

$$
D_{0}^{-1}=\left[\begin{array}{cc}
d_{11}^{-1} & 0 \\
-d_{22}^{-1} d_{21} d_{11}^{-1} & d_{22}^{-1}
\end{array}\right]
$$

Therefore the first diagonal block of $\left(D_{0}^{\top} S^{-1} D_{0}\right)^{-1}$ equals $d_{11}^{-1} S_{11}\left(d_{11}^{-1}\right)^{\top}$. Similarly, the first block of $\left(D_{0}^{\top}\left(S^{\mathcal{A}}\right)^{-1} D_{0}\right)^{-1}$ can be written as $^{6}$

$$
d_{11}^{-1} S_{11}^{\mathcal{A}}\left(d_{11}^{-1}\right)^{\top}=\lambda d_{11}^{-1} S_{11}\left(d_{11}^{-1}\right)^{\top} .
$$

This shows that asymptotic standard errors for the estimates of $\theta_{1}$ shrink by a factor of $1-\sqrt{\lambda}$ when the adjusted-moment estimator is used rather than the short estimator. Because the overidentified estimator is asymptotically equivalent to the adjusted-moment estimator, the shrinkage is the same.

\footnotetext{
${ }^{6}$ Recall that $D_{0}^{\mathcal{A}}=D_{0}$.
} 
It is more interesting to look at the effect on the standard errors of the second set of parameters $\theta_{2}$. The second diagonal block of $\left(D_{0}^{\top}\left(S^{\mathcal{A}}\right)^{-1} D_{0}\right)^{-1}$ reduces to

$$
\begin{aligned}
\left(D_{0}^{\top}\left(S^{\mathcal{A}}\right)^{-1} D_{0}\right)_{22}^{-1}=d_{22}^{-1}\left[d_{21} d_{11}^{-1} S_{11}^{\mathcal{A}}-S_{21}^{\mathcal{A}}\right]\left(S_{11}^{\mathcal{A}}\right)^{-1}\left[d_{21} d_{11}^{-1} S_{11}^{\mathcal{A}}-S_{21}^{\mathcal{A}}\right]\left(d_{22}^{-1}\right)^{\top} & \\
& +d_{22}^{-1}\left[S_{22}^{\mathcal{A}}-S_{21}^{\mathcal{A}}\left(S_{11}^{\mathcal{A}}\right)^{-1} S_{12}^{\mathcal{A}}\right]\left(d_{22}^{-1}\right)^{\top}
\end{aligned}
$$

Thus the variance for the second set of variables can be decomposed into two parts. The first part represents the effect of the first moment conditions on the second variables. The second part represents the variance due only to the residual variance of the second set of moment conditions: $S_{22}^{\mathcal{A}}-S_{21}^{\mathcal{A}}\left(S_{11}^{\mathcal{A}}\right)^{-1} S_{12}^{\mathcal{A}}$ is the variance-covariance matrix of the second set of moment conditions conditional on the first. The second diagonal block of $\left(D_{0}^{\top} S D_{0}\right)_{22}^{-1}$, which gives the standard errors for $\theta_{2}$ under standard GMM, has an analogous decomposition.

Adding the new data reduces the first term in (34), just as it reduces the asymptotic variance of $\theta_{1}$ :

$$
\left[d_{21} d_{11}^{-1} S_{11}^{\mathcal{A}}-S_{21}^{\mathcal{A}}\right]\left(S_{11}^{\mathcal{A}}\right)^{-1}\left[d_{21} d_{11}^{-1} S_{11}^{\mathcal{A}}-S_{21}^{\mathcal{A}}\right]=\lambda\left[d_{21} d_{11}^{-1} S_{11}-S_{21}\right] S_{11}^{-1}\left[d_{21} d_{11}^{-1} S_{11}-S_{21}\right]
$$

However the second term in (34) does not change with the addition of new data, not surprisingly because it represents the variance of the second moment conditions conditional on the value of the first:

$$
S_{22}^{\mathcal{A}}-S_{21}^{\mathcal{A}}\left(S_{11}^{\mathcal{A}}\right)^{-1} S_{12}^{\mathcal{A}}=S_{22}-S_{21} S_{11}^{-1} S_{12}
$$

Thus the decrease in the standard errors depends on the extent to which the first term dominates the second term. For example, when the second set of moments are perfectly correlated with the first set, the residual variance is zero,

$$
S_{22}-S_{21} S_{11}^{-1} S_{12}=0
$$

and the standard errors for $\theta_{2}$ also shrink by a factor of $1-\sqrt{\lambda}$. At the other extreme, suppose that $f_{2}$ tells you nothing about $\theta_{2}$, i.e. $d_{21}=0\left(\theta_{1}\right.$ does not enter into $\left.f_{2}\right)$ and $S_{21}=S_{12}^{\top}=0$ (the moment conditions are independent). Then the inclusion of the longer series leads to no shrinkage in the asymptotic variance of $\theta_{2}$.

Of course, even if the two sets of moment conditions are independent $\left(S_{21}=S_{12}^{\top}=0\right)$, the sampling variance of $\theta_{2}$ may still fall because the sampling variance of $\theta_{1}$ is reduced. As long as $d_{21} \neq 0$, the first term in (34) is nonzero and there is an effect on the standard errors of $\theta_{2}$. 
Similarly, even if there is no impact of $\theta_{1}$ on the second set of moment conditions $\left(d_{21}=0\right)$ the first set of moment conditions help to estimate $\theta_{2}$ if the covariance between the two moment conditions is nonzero.

\section{An illustration with mutual fund data}

This section briefly illustrates our methods in an application to mutual fund performance evaluation. Section 5.1 describes the model for normal returns; mutual fund skill is defined relative to this model. Section 5.2 describes the data, Section 5.3 the moment conditions used in the estimation, and Section 5.4 the results.

\subsection{Conditional factor model}

We start by assuming a conditional beta pricing model of the form

$$
E_{t}\left[r_{t+1}\right]=E_{t}\left[r_{p, t+1}\right]^{\top} \beta_{t},
$$

where $\beta_{t}$ is a column vector equal to

$$
\beta_{t}=\operatorname{Var}_{t}\left(r_{p, t+1}\right)^{-1} \operatorname{Cov}_{t}\left(r_{p, t+1}, r_{t+1}\right),
$$

and $r_{p, t+1}$ is a $K \mathrm{x} 1$ column vector of returns on zero-cost benchmark portfolios. In what follows, we will denote excess returns using lower-case $r$; gross returns will be denoted $R$. Shanken (1990) discusses estimation and testing of conditional factor models. Ferson and Schadt (1996) discuss the importance of measuring mutual fund performance relative to conditional factor models as opposed to unconditional models.

As shown in Cochrane (2001), (36) is equivalent to specifying a conditional stochastic discount factor (SDF) model in which the SDF is linear in $r_{p}$ with coefficients that are elements of the time- $t$ information set. Let $Z_{t}$ denote the variable, or vector of variables, in the investor's information set at time $t$. Then the linear specification implies

$$
M_{t+1}=a\left(Z_{t}\right)+b\left(Z_{t}\right)^{\top} r_{p, t+1} .
$$

Any return $R_{t+1}$ that is correctly priced by the SDF, $M_{t+1}$, satisfies

$$
E_{t}\left[R_{t+1} M_{t+1}\right]=1
$$

where $E_{t}$ is taken to mean the expectation conditional on the value of $Z_{t}$. Following Cochrane (2001) and Lettau and Ludvigson (2001), we make the further assumption that the coefficients are 
linear functions of $Z_{t}$, and, for notational convenience, we assume a single information variable. ${ }^{7}$ With these assumptions, the stochastic discount factor associated with the conditional factor model is given by

$$
M_{t+1}=a_{0}+a_{1} Z_{t}+\left(b_{0}+b_{1} Z_{t}\right)^{\top} r_{p, t+1}
$$

Let $R_{f, t+1}$ denote the riskfree rate of return. Because $R_{f, t+1}$ is known at time $t$,

$$
E_{t}\left[M_{t+1}\right]=\frac{1}{R_{f, t+1}}
$$

Zero-cost portfolios, or returns in excess of the riskfree rate satisfy

$$
E_{t}\left[r_{t+1} M_{t+1}\right]=0
$$

Condition (41) and the specification (39) imply that for any asset with return $r_{t+1}$,

$$
E_{t}\left[r_{t+1}\right]=-\frac{\left(b_{0}+b_{1} Z_{t}\right)^{\top}}{E_{t}\left[M_{t+1}\right]} \operatorname{Cov}_{t}\left(r_{p, t+1}, r_{t+1}\right) .
$$

Because $M_{t+1}$ must price the reference assets correctly, (42) holds for the reference assets, and

$$
\frac{\left(b_{0}+b_{1} Z_{t}\right)^{\top}}{E_{t}\left[M_{t+1}\right]}=-E_{t}\left[r_{p, t+1}\right]^{\top} \operatorname{Var}_{t}\left(r_{p, t+1}\right)^{-1}
$$

Substituting (43) in to (42) produces (36). Thus specifying the stochastic discount factor as (39) implies a conditional beta pricing model.

Now consider the return on a fund $r_{i, t+1}$ and suppose that this return can be described by

$$
E_{t}\left[r_{i, t+1}\right]=\alpha_{i}+E_{t}\left[r_{p, t+1}\right]^{\top} \beta_{i, t+1},
$$

where $\alpha_{i}$ represents abnormal performance relative to the conditional factor model described in (36). ${ }^{8}$ It follows that mutual fund returns must satisfy

$$
E_{t}\left[M_{t+1}\left(r_{i, t+1}-\alpha_{i}\right)\right]=0
$$

Using the linear specification for the SDF in (39), we obtain the following:

$$
E_{t}\left[\left(a_{0}+a_{1} Z_{t}+\left(b_{0}+b_{1} Z_{t}\right)^{\top} r_{p, t+1}\right)\left(r_{i, t+1}-\alpha_{i}\right)\right]=0
$$

\footnotetext{
${ }^{7}$ The linearity assumption can be easily relaxed by allowing the coefficients to be polynomials in the information variable. Likewise, it is easy to relax the assumption of a single information variable.

${ }^{8}$ It is also possible to allow $\alpha_{i}$ to be a function of the information variable, so performance itself is conditional. Lynch, Wachter, and Boudry (2004) empirically investigate conditional performance using the estimators proposed in the present paper.
} 
Equation (46) applied to fund returns, (38) applied to the riskfree rate, and (41) applied to the factor returns form the basis for our estimation. We also consider performance relative to an unconditional factor model:

$$
E\left[r_{t+1}\right]=E\left[r_{p, t+1}\right]^{\top} \beta
$$

with

$$
\beta=\operatorname{Var}\left(r_{p, t+1}\right)^{-1} \operatorname{Cov}\left(r_{p, t+1}, r_{t+1}\right) .
$$

In this case, the equivalent stochastic discount factor is given by

$$
M_{t+1}=a+b^{\top} r_{p, t+1}
$$

and unconditional versions of (38) (applied to the riskfree rate) and (41) (applied to factor returns) are used in the estimation.

\subsection{Data}

In our implementation, we focus on the case where the information variable is equal to the dividendyield. ${ }^{9}$ We consider a model for benchmark returns with four factors: the excess market return, the book-to-market return (HML), the small minus big market capitalization return (SMB), and the return on a momentum factor (UMD). The role of the first three factors in explaining the crosssection of returns is demonstrated by Fama and French (1993), while the last factor is introduced by Carhart (1997) who demonstrates its importance in mutual fund performance evaluation. The riskfree rate and returns on these factors can be found on the website of Ken French. Descriptions of the construction of these portfolios can be found in Fama and French (1993), and on the website. The data used to construct the information variable, the 12-month dividend yield on the valueweighted NYSE, come from CRSP. The data are available starting in January 1927.

The mutual fund data are from Elton, Gruber, and Blake (1996). Their sample consists of monthly returns on the 188 common stock funds in the 1977 edition of Wiesenberger's Investment Companies that have total net assets of $\$ 15$ million or more and that are not restricted. The data run from January 1977 through until December 1993. Four fund type groups are constructed using the Wiesenberger style categories, with our classifications always consistent with those employed by Elton, Gruber and Blake and Ferson and Schadt (1996). Three of the four fund types are the Wiesenberger categories: maximum capital gain, growth, and growth and income. The fourth

\footnotetext{
${ }^{9}$ The dividend-yield is used because, for theoretical reasons, it would be expected to contain information about future returns (Campbell and Shiller (1988)), and because it has been shown to predict future returns in the data (Fama and French (1989)).
} 
group includes all other funds in our sample and is referred to as miscellaneous. For disappearing funds, returns are included through until disappearance so the fund-type returns do not suffer from survivor conditioning. Funds are reclassified at the start of each year based on their category at that time.

\subsection{Moment conditions}

Following Hansen and Singleton (1982), we use of the law of iterated expectations to estimate the $2(K+1)$ SDF parameters of the conditional model. The moment conditions are

$$
E\left[\left(\left(a_{0}+a_{1} Z_{t}\right)+\left(b_{0}+b_{1} Z_{t}\right)^{\top} r_{p, t+1}\right)\left[\begin{array}{c}
R_{f, t+1} \\
r_{p, t+1}
\end{array}\right] \otimes\left[\begin{array}{c}
1 \\
Z_{t}
\end{array}\right]-\left[\begin{array}{l}
1 \\
0
\end{array}\right] \otimes\left[\begin{array}{c}
1 \\
Z_{t}
\end{array}\right]\right]=0
$$

These must hold if the SDF in (39) correctly prices the riskfree return and the zero-cost factor portfolio returns. As Hansen and Singleton show, these moment conditions are obtained by multiplying both sides of (38) and (41) by 1 and by $Z_{t}$ and using the law of iterated expectations. Because there are $2(K+1)$ moment conditions, the system is exactly identified. To estimate fund-type performance, (46) is used with excess fund-type returns. Applying the law of iterated expectations to $(46)$ results in

$$
E\left[\left(r_{i, t+1}-\alpha_{i}\right)\left(\left(a_{0}+a_{1} Z_{t}\right)+\left(b_{0}+b_{1} Z_{t}\right)^{\top} r_{p, t+1}\right)\right]=0
$$

Because there is a single moment condition and a single parameter, fund-type performance is also exactly identified. ${ }^{10}$

Because the fund data begin in January 1977, but the factor return and dividend-yield data begin in January 1927, the setting is that of Section 2. Moreover, because the moment conditions (47) exactly identify the SDF parameters and (48) exactly identifies the fund parameters, the results of Section 4 also apply. Let $r_{N}$ denote the $N \times 1$ vector of returns on fund styles, (in our analysis, $N=4$ ), and let $\alpha_{N}$ denote the $N \times 1$ vector of outperformance parameters $\alpha_{i}$. In the notation

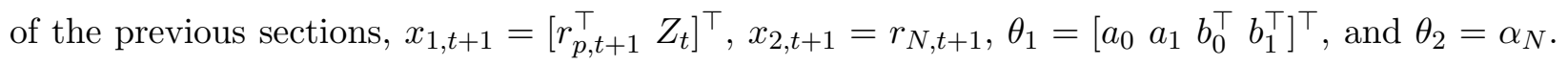
Define

$$
\begin{aligned}
f_{1}\left(x_{1, t+1}, \theta_{1}\right) & =\left[\begin{array}{c}
R_{f, t+1} \\
r_{p, t+1}
\end{array}\right]\left(\left(a_{0}+a_{1} Z_{t}\right)+\left(b_{0}+b_{1} Z_{t}\right)^{\top} r_{p, t+1}\right) \otimes\left[\begin{array}{l}
1 \\
Z_{t}
\end{array}\right]-\left[\begin{array}{l}
1 \\
0
\end{array}\right] \otimes\left[\begin{array}{c}
1 \\
Z_{t}
\end{array}\right] \\
f_{2}\left(x_{t+1}, \theta\right) & =\left(r_{N, t+1}-\alpha_{N}\right)\left(\left(a_{0}+a_{1} Z_{t}\right)+\left(b_{0}+b_{1} Z_{t}\right)^{\top} r_{p, t+1}\right)
\end{aligned}
$$

\footnotetext{
${ }^{10}$ The stochastic discount factor parameters can be over-identified by multiplying by nonlinear functions of $Z_{t}$ in (47). Likewise, multiplying the term inside the expectation in (48) by functions of $Z_{t}$ would over-identify the fund parameter $\alpha_{i}$. For simplicity, we consider only the exactly identified systems here.
} 
where $\otimes$ denotes the Kronecker product. Recall that $x_{t}=\left[\begin{array}{ll}x_{1 t}^{\top} & x_{2 t}^{\top}\end{array}\right]^{\top}$ and $\theta=\left[\theta_{1}^{\top} \theta_{2}^{\top}\right]^{\top}$. To estimate the unconditional SDF, (49) and (50) are replaced by

$$
\begin{aligned}
f_{1}\left(x_{1, t+1}, \theta_{1}\right) & =\left[\begin{array}{c}
R_{f, t+1} \\
r_{p, t+1}
\end{array}\right]\left(a+b^{\top} r_{p, t+1}\right)-\left[\begin{array}{l}
1 \\
0
\end{array}\right] \\
f_{2}\left(x_{2, t+1}, \theta\right) & =\left(r_{N, t+1}-\alpha_{N}\right)\left(a+b^{\top} r_{p, t+1}\right) .
\end{aligned}
$$

Let $\hat{S}_{i j, \lambda T}(\tilde{\theta})$ be the Newey and West (1987) estimator of $S_{i j}$ that uses the short sample, 6 lags and parameter estimate $\tilde{\theta}$. If $\tilde{\theta}$ is a consistent estimate of $\theta_{0}$, the results of Newey and West imply that $\hat{S}_{i j, \lambda T}(\tilde{\theta})$ is a consistent estimator of $S_{i j}$. The moments for the adjusted-moment estimator can then be defined by (10), with $f_{1}$ and $f_{2}$ defined as above, and with $\hat{B}_{21, \lambda T}$ given by

$$
\hat{B}_{21, \lambda T}(\tilde{\theta})=\hat{S}_{21, \lambda T}(\tilde{\theta})\left(\hat{S}_{11, \lambda T}(\tilde{\theta})\right)^{-1}
$$

To obtain a consistent estimate $\tilde{\theta}$, we first estimate the system via standard GMM. We then use this first-stage estimate to obtain $\hat{B}_{21, \lambda T}(\tilde{\theta})$ for use in the second stage. Second stage adjusted-moment estimates are reported.

The over-identified estimator is given by (11). To completely specify this estimator, it is necessary to specify a consistent estimator for $S^{\mathcal{I}}$, which is then used as the inverse of the weighting matrix. The identity matrix is used to obtain first-stage over-identified estimates; these estimates become the inputs $\tilde{\theta}$ into the Newey-West estimator of $S^{\mathcal{I}}$ to obtain the second-stage estimate. Then the second-stage estimates are used to obtain third-stage over-identified estimates. This procedure is repeated until convergence.

When calculating standard errors for the over-identified or adjusted-moment estimations, NeweyWest evaluated at the parameter estimates for that iteration are used. For the short estimation, standard errors are calculated two ways: using Newey-West evaluated at the parameter estimates reported for the over-identified estimation and for the adjusted-moment estimation. Doing so allows the efficiency gains from using the adjusted-moment or over-identified estimators instead of the short estimator to be quantified more easily.

\subsection{Results}

Table 1 reports abnormal performance estimates for the Carhart (1997) four-factor model. Panel A reports the abnormal performance parameter $(\alpha)$ for each fund type when the factor model is conditional, while Panel B reports $\alpha$ for each fund type when the factor model is unconditional. There are five columns in the table. The first column reports the estimates and standard errors 
using the adjusted-moment method and the second the over-identified method. The third column reports the results using standard GMM (short method). As discussed above, standard errors for the short method are evaluated at parameter estimates from the longer sample. One standard error uses the Newey-West technique evaluated at the adjusted-moment estimates, while the other uses the Newey-West technique evaluated at the over-identified estimates. The last column averages the percent reduction in the standard error due to using the adjusted-moment estimator rather than short and the over-identified estimator rather than short.

Panels A and B show that the estimates of performance differ little between the adjustedmoment, over-identified, and short methods, and between the conditional and unconditional models. Point estimates for performance of growth funds are higher than the estimates for other types of funds. However, the individual estimates are not significantly different from zero. The standard errors differ markedly between the methods that use the full sample and standard GMM. For example, under the conditional model, the $\alpha$ on growth funds is estimated to be $.06 \%$ per month for all three methods. For the short method, the standard error is 0.23. In contrast, when the adjusted-moment or over-identified method is used, the standard error falls to 0.12 , a reduction of $48 \%$. The reduction for the miscellaneous category is $28 \%$, while the reductions for maximal capital gain and growth and income are $48 \%$ and $49 \%$ respectively. For performance relative to the unconditional model, the reductions are qualitatively similar.

Table 2 reports SDF estimates. Panel A reports results for the conditional model; Panel B reports results for the unconditional model. The organization is the same as in Table 1, except that the last column, \% reduction, is omitted. As the table shows, estimates of the SDF parameters are similar for the adjusted-moment and over-identified estimators, but different for the short estimator. Clearly, inference about these parameters is altered when the data set is expanded to include all the available observations. Many studies of stock returns use the longer data when possible (see, e.g. Kandel and Stambaugh (1996)); our method allows the advantages of using the longer data to be retained, even in a setting where the estimation involves data not observed for the whole interval. As noted in Section 4, standard errors on parameters that can be estimated from the full data set fall by $1-\sqrt{\lambda}$. In this example, $\lambda=204 / 804$. Thus the standard errors on the SDF parameters fall by $49.6 \%$.

Table 1 shows that the standard errors on the mutual fund parameters fall substantially, often by nearly as much as the standard errors on the SDF parameters. The standard errors on the mutual fund parameters fall for two reasons, as explained in Section 4. First, moment conditions identifying 
Table 1: Fund-type performance relative to the Carhart 4-factor model

Estimates of the abnormal performance parameter $\alpha$ when the SDF model is conditional (Panel A), and when it is unconditional (Panel B), for each of the four fund types. The factors are the excess return on the market portfolio and the returns on the SMB, HML, and momentum (UMD) portfolios. Abnormal performance parameters calculated using the adjusted-moment method (AM), over-identified method (OI), and standard GMM (Short), are reported. Standard errors for Short are calculated using AM or OI estimates. \% Reduction is the average percent reduction in standard error due to using AM and OI rather than their Short counterparts. Data are monthly, beginning in 1927 and ending in 1993. Short sample data cover the period 1977 to 1993 . Newey-West standard errors calculated with 6 lags are in parentheses. Returns are in \% per month.

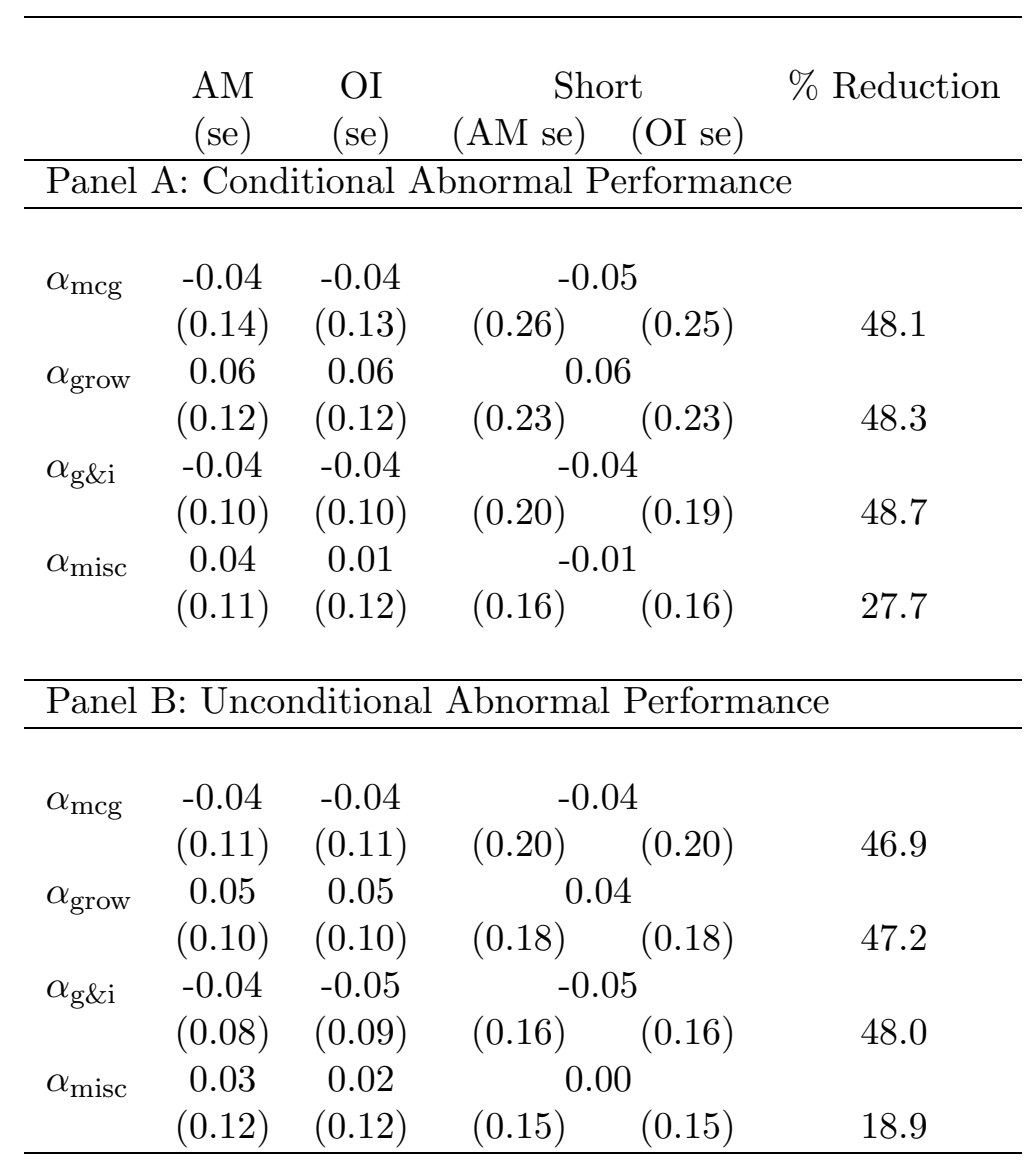


Table 2: Stochastic discount factor estimates for the Carhart 4-factor model

Estimates of SDF parameters when the SDF model is conditional (Panel A), and when it is unconditional (Panel B). The factors are the excess return on the market portfolio and the returns on the SMB, HML, and momentum (UMD) portfolios. SDF parameters calculated using the Adjusted Moment method (AM), Over-Identified method (OI), and standard GMM (Short), are reported. Standard errors for Short are calculated using AM or OI estimates. In all cases, the \% reduction in standard errors is 49.6\%. Data are monthly, beginning in 1927 and ending in 1993. Short sample data cover the period 1977 to 1993. Newey-West standard errors calculated with 6 lags are in parentheses.

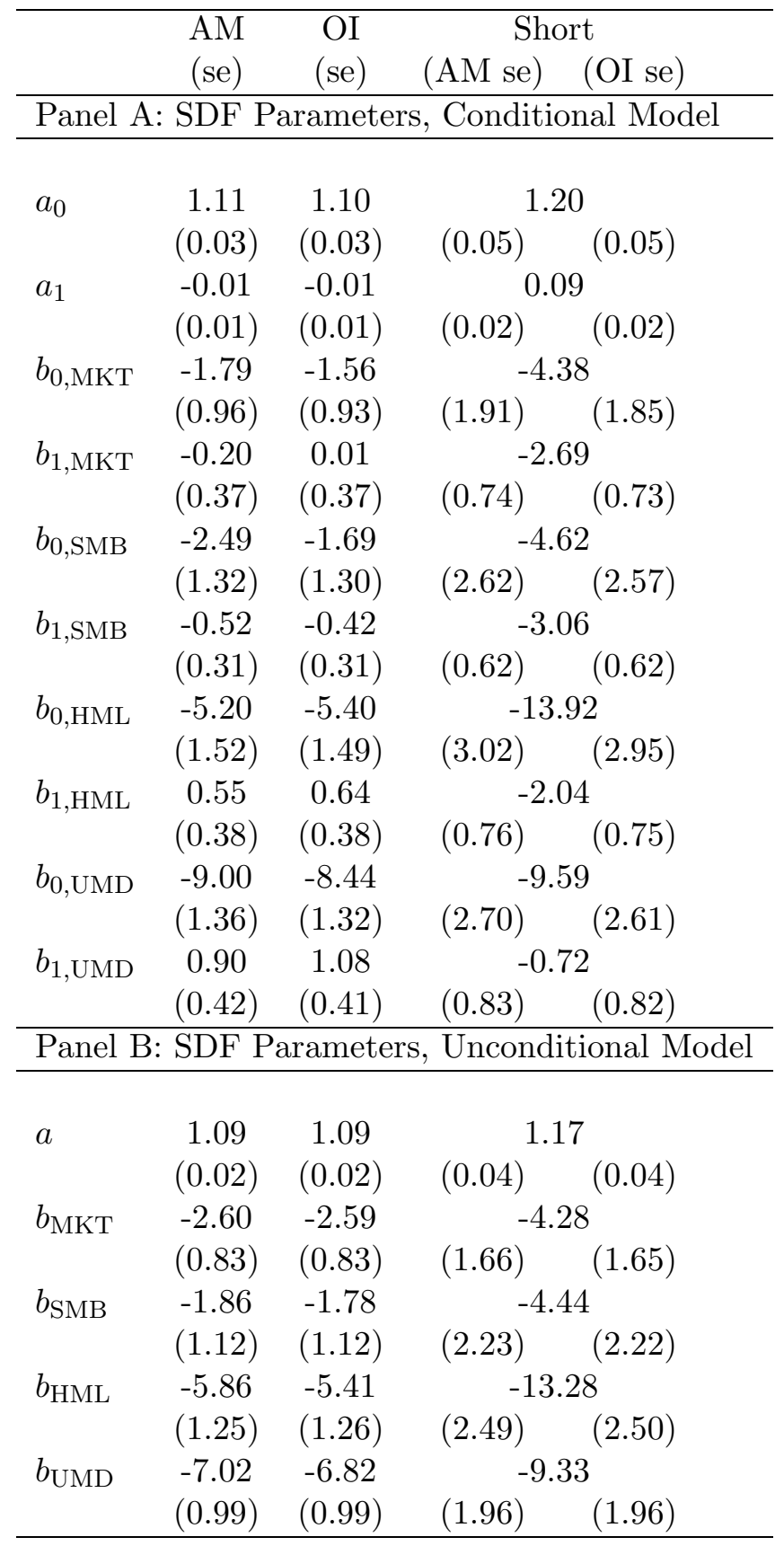


performance are correlated with moment conditions identifying the SDF. Our estimators make use of this correlation to lower the variance on the conditions identifying the mutual fund parameters, even though no extra data on mutual funds are used. Second, the SDF parameters appear in both sets of moment conditions. When the additional data are used, more precise estimates of these parameters enter into the conditions for abnormal performance. In the notation of Section 4, both $S_{21} \neq 0$ and $d_{21} \neq 0$. The standard errors on the fund parameters could fall by at most $49.6 \%$, the percent reduction in the SDF standard errors. The fact that the reductions reported in Table 1 are close to this indicates that the effects of introducing the longer sample can be large.

\section{Extensions}

The previous sections considered cases where there were two relevant sample periods: a "short" sample period over which all data are observed, and a "long" sample period over which only some of the data are observed. This section extends the methods to cases where there are more than two different sample periods. In order to extend the estimators of Section 2, it is necessary to prove a theorem analogous to Theorem 1.1 for the case where the data of length $T$ is divided into more than two blocks. Let $\eta_{1}, \eta_{2}, \ldots, \eta_{n}$ denote rational numbers such that $\sum_{k=1}^{n} \eta_{k}=1$. Let $n_{0}$ be the smallest integer such that the product with $\eta_{j}$ is an integer, for all $j$. As above, we will restrict attention to values $T$ that are a multiple of $n_{0}$. Define the following partial sums of $g$ :

$$
\begin{aligned}
g_{\eta_{1} T}(\theta) & =\frac{1}{\eta_{1} T} \sum_{t=1}^{\eta_{1} T} f\left(x_{t}, \theta\right) \\
g_{\eta_{j} T}(\theta) & =\frac{1}{\eta_{j} T} \sum_{t=\left(\eta_{1}+\cdots+\eta_{j-1}\right) T+1}^{\left(\eta_{1}+\cdots+\eta_{j}\right) T} f\left(x_{t}, \theta\right), \quad j=2, \ldots, n .
\end{aligned}
$$

Theorem 6.1 Define $g_{\eta_{1} T}$ as in (52) and $g_{\eta_{j} T}$ as in (53) for $j=2, \ldots, n$. Assumptions 1-5 imply

$$
\sqrt{T}\left(\begin{array}{c}
\sqrt{\eta_{1}} g_{\eta_{1} T}\left(\theta_{0}\right) \\
\sqrt{\eta_{2}} g_{\eta_{2} T}\left(\theta_{0}\right) \\
\vdots \\
\sqrt{\eta_{n}} g_{\eta_{n} T}\left(\theta_{0}\right)
\end{array}\right) \rightarrow_{\mathrm{d}} N\left(0,\left[\begin{array}{cccc}
S & 0 & \ldots & 0 \\
0 & S & \ldots & 0 \\
0 & 0 & \ddots & 0 \\
0 & 0 & 0 & S
\end{array}\right]\right)
$$

as $T \rightarrow \infty$.

Proof We proceed by induction. The case for $n=1$ follows from standard results (e.g. White and Domowitz (1984, Theorem 2.4)). Suppose (54) holds for $n-1$. Because $x_{t}$ is stationary, we can 


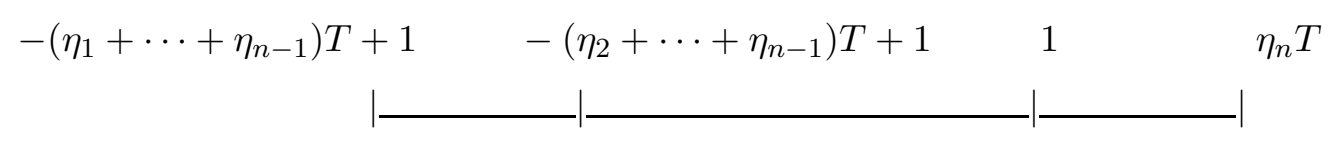

Figure 2: Numbering scheme used in the proof of Theorem 6.1

define a new set of partial sums $\tilde{g}$ with the same joint distribution as the partial sums $g$. Let

$$
\tilde{g}_{\eta_{n} T}\left(\theta_{0}\right)=\frac{1}{\eta_{n} T} \sum_{t=1}^{\eta_{n} T} f\left(x_{t}, \theta\right)
$$

while

$$
\tilde{g}_{\eta_{j} T}\left(\theta_{0}\right)=\sum_{-\left(\eta_{j}+\ldots \eta_{n-1}\right) T+1}^{-\left(\eta_{j+1}+\ldots \eta_{n-1}\right) T} f\left(x_{t}, \theta\right), \quad j=1, \ldots, n-1 .
$$

As Figure 2 illustrates, the start data of new "sample" is $-\left(\eta_{1}+\cdots+\eta_{n-1}\right) T+1$ while the end date is $\eta_{n} T$. Then $\tilde{g}_{\eta_{1} T}\left(\theta_{0}\right), \ldots, \tilde{g}_{\eta_{n} T}\left(\theta_{0}\right)$ have the same joint distribution as $g_{\eta_{1} T}\left(\theta_{0}\right), \ldots, g_{\eta_{n} T}\left(\theta_{0}\right)$.

By Lemma 1.2, for any $1 \times l$ vectors $\mu_{1}, \ldots, \mu_{n}$ such that $\mu_{j} \mu_{j}^{\top}=1$, and scalars $c_{1}, \ldots, c_{n}$,

$$
\begin{gathered}
\lim _{T \rightarrow \infty} P\left(\sqrt{\eta_{n} T} \mu_{n} S^{-1} \tilde{g}_{\eta_{n} T}\left(\theta_{0}\right)<c_{n}, \sqrt{\eta_{n-1} T} \mu_{n-1} S^{-1} \tilde{g}_{\eta_{n-1} T}\left(\theta_{0}\right)<c_{n-1}, \ldots, \sqrt{\eta_{1} T} \mu_{1} S^{-1} \tilde{g}_{\eta_{1} T}\left(\theta_{0}\right)<c_{1}\right)= \\
\lim _{T \rightarrow \infty} P\left(\sqrt{\eta_{n} T} \mu_{n} S^{-1} \tilde{g}_{\eta T}\left(\theta_{0}\right)<c_{n}\right) \times \\
\lim _{T \rightarrow \infty} P\left(\sqrt{\eta_{n-1} T} \mu_{n-1} S^{-1} \tilde{g}_{\eta_{n-1} T}\left(\theta_{0}\right)<c_{n-1}, \ldots, \sqrt{\eta_{1} T} \mu_{1} S^{-1} \tilde{g}_{\eta_{1} T}\left(\theta_{0}\right)<c_{1}\right) .
\end{gathered}
$$

The result then follows from the induction assumption and asymptotic normality of $\sqrt{\eta_{n} T} g_{\eta_{n} T}$.

\subsection{Extending the Over-Identified Estimator}

An advantage of the over-identified estimator is that it is has a transparent extension to samples where there is a more general pattern of missing data. Theorem 6.1 gives the joint distribution of partial sums of $g$. We now use this result to extend the over-identified estimator.

As before, we consider the situation where not all moments are observed over the whole sample. Here, however, we allow for an arbitrary number of missing "blocks" of data, and they can occur anywhere in the sample, rather than simply at the beginning. Our asymptotic results will keep the size of these missing blocks of data proportional to the size of the overall sample, so that the missing data problem does not become trivial, just as in the case where there were data missing at the start of the sample.

Consider intervals of the data defined by points in time where at least one sample moment starts or ends. Say these points in time divide the sample up into disjoint intervals $1, \ldots, n$. These 
intervals can be weakly ordered by how many of the sample moments are observed over that interval. That is, $\pi_{1}$ sample moments are observed over the first segment, $\pi_{2} \leq \pi_{1}$ over the second segment, etc. Let $\lambda_{1}$ denote the ratio of the length of the first region (the region over which the greatest number of moments are observed) to the length of the entire sample, $\lambda_{2}$ the ratio of the length of the second region to the length of the entire sample, etc. Then $\lambda_{1}, \ldots, \lambda_{n}$ can be thought of in the same way as $\eta_{1}, \ldots, \eta_{n}$ in the previous section, except that while the $\eta$ s are labeled according to their order in the sample, the $\lambda$ s are labeled according to how many data moments are observed over that segment. Note that $\sum_{i=1}^{n} \lambda_{i}=1$.

Define points $t_{1}, \ldots t_{n}$ so that the first data segment begins at $t_{1}+1$, the second data segment at $t_{2}+1$, etc. Then

$$
g_{\lambda_{j} T}(\theta)=\frac{1}{\lambda_{j} T} \sum_{t=t_{j}+1}^{t_{j}+\lambda_{j} T} f\left(x_{t}, \theta\right), \quad j=1, \ldots, n .
$$

For the case described in Section 2, the first segment consist of points $(1-\lambda) T+1$ to $T$. All moments were observed over this segment. The second segment consists of points 1 to $(1-\lambda) T$. Only a subset of moments are observed over these points. In this example, $t_{1}=(1-\lambda) T, t_{2}=0$, $\lambda_{1}=\lambda$, and $\lambda_{2}=(1-\lambda)$. We adopt the same notational convention as in Section 2: $\lambda_{j} T$ will refer to the length of the segment between $t_{j}+1$ and $t_{j}+\lambda_{j} T$, and the segment itself.

Finally, let $\phi_{i}$ denote the set of data series that are observed in data segment $\lambda_{i}$. Define

$$
f_{\phi_{j}}\left(x_{t}, \theta\right)=\left(f_{i_{1}}\left(x_{t}, \theta\right), \ldots, f_{i_{\pi_{j}}}\left(x_{t}, \theta\right)\right)^{\top}
$$

where $\left\{i_{1}, \ldots, i_{\pi_{j}}\right\} \in \phi_{j}$ and $i_{1}<\cdots<i_{\pi_{j}}$. Then $f_{\phi_{j}}$ are the components of $f$ observed over the segment $\lambda_{j} T$. Similarly, define the $\pi_{j} \times q$ matrix

$$
D_{0, \phi_{j}}=E\left[\left.\frac{\partial f_{\phi_{j}}}{\partial \theta}\right|_{\theta_{0}}\right]=\left(D_{0, i_{1}}^{\top}, \ldots, D_{0, i_{\pi_{j}}}^{\top}\right)^{\top}
$$

the $\pi_{j} \times 1$ vector

$$
g_{\phi_{j}, \lambda_{j} T}(\theta)=\frac{1}{\lambda_{j} T} \sum_{t=t_{j}+1}^{t_{j}+\lambda_{j} T} f_{\phi_{j}}\left(x_{t}, \theta\right)
$$

and the $\pi_{j} \times \pi_{j}$ matrices

$$
R_{\phi_{j}}(\tau)=E\left[f_{\phi_{j}}\left(x_{0}, \theta_{0}\right) f_{\phi_{j}}\left(x_{-\tau}, \theta_{0}\right)^{\top}\right]
$$

and

$$
S_{\phi_{j}}=\sum_{\tau=-\infty}^{\infty} R_{\phi_{j}}(\tau)
$$


The extended over-identified estimator, for the case where there are $n$ blocks of data and the total data length is $T$, has moment conditions

$$
h_{T}^{\mathcal{I}_{n}}(\theta)=\left[g_{\phi_{1}, \lambda_{1} T}(\theta)^{\top}, g_{\phi_{2}, \lambda_{2} T}(\theta)^{\top}, \ldots, g_{\phi_{n}, \lambda_{n} T}(\theta)^{\top}\right]^{\top}
$$

for $\theta \in \Theta$. The $\mathcal{I}_{n}$ superscript refers to the fact that these are moment conditions for the overidentified estimator, and that there are $n$ non-overlapping intervals. The $T$ subscript refers to the fact that the data length is $T .{ }^{11}$ As in Section $2, \sqrt{T} h_{T}^{\mathcal{I}_{n}}(\theta)$ is asymptotically normally distributed. The following is analogous to Theorem 2.1.

Theorem 6.2 Assumptions 1-5 imply

$$
\sqrt{T} h_{T}^{\mathcal{I}_{n}}\left(\theta_{0}\right) \rightarrow_{\mathrm{d}} N\left(0, S^{\mathcal{I}_{n}}\right)
$$

where

$$
S^{\mathcal{I}_{n}}=\left[\begin{array}{cccc}
\frac{1}{\lambda_{1}} S_{\phi_{1}} & 0 & \cdots & 0 \\
0 & \frac{1}{\lambda_{2}} S_{\phi_{2}} & \cdots & 0 \\
0 & 0 & \ddots & 0 \\
0 & 0 & 0 & \frac{1}{\lambda_{n}} S_{\phi_{n}}
\end{array}\right]
$$

as $T \rightarrow \infty$.

The extended over-identified estimator takes (55) as moment conditions. In principle, any weighting matrix can be used, but we will emphasize the case when the weighting matrix converges almost surely to (56). Define

$$
\hat{\theta}_{T}^{\mathcal{I}_{n}}=\operatorname{argmin}_{\theta} h_{T}^{\mathcal{I}_{n}}(\theta)^{\top} W_{T}^{\mathcal{I}_{n}} h_{T}^{\mathcal{I}_{n}}(\theta) .
$$

Not surprisingly, the same consistency and asymptotic efficiency results go through for the extended over-identified estimator as for the original over-identified estimator. Here, we repeat the results but omit the proofs, which follow along the same lines as the corresponding proofs in Section 2.

Assumption 10 The weighting matrix $W_{T}^{\mathcal{I}_{n}}$ converges almost surely to a positive-definite matrix $W^{\mathcal{I}_{n}}$.

Theorem 6.3 Assumptions $1-5$ and 10 imply that as $T \rightarrow \infty, \hat{\theta}_{T}^{\mathcal{I}_{n}} \rightarrow$ a.s. $\theta_{0}$.

\footnotetext{
${ }^{11}$ This notation does not, of course, completely define the over-identified estimator. For that, one would need the points at which the data intervals begin, $t_{1}, \ldots, t_{n}$. These points in turn depend in a complicated way on $\lambda_{1}, \ldots, \lambda_{n}$ and $T$.
} 
Define

$$
D_{0}^{\mathcal{I}_{n}}=\left[\begin{array}{llll}
D_{0, \phi_{1}}^{\top} & D_{0, \phi_{2}}^{\top} & \ldots & D_{0, \phi_{n}}^{\top}
\end{array}\right]^{\top} .
$$

Note that Assumptions 1-5 imply that

$$
D_{0}^{\mathcal{I}_{n}}=\left.\lim _{T \rightarrow \infty} \frac{\partial h_{T}^{\mathcal{I}_{n}}}{\partial \theta}\right|_{\theta_{0}} .
$$

Theorem 6.4 Assumptions 1-5, and 7-10 imply that as $T \rightarrow \infty$,

$$
\sqrt{T}\left(\hat{\theta}_{T}^{\mathcal{I}_{n}}-\theta_{0}\right) \rightarrow{ }_{\mathrm{d}} N\left(0,\left(\left(D_{0}^{\mathcal{I}_{n}}\right)^{\top} W^{\mathcal{I}_{n}} D_{0}^{\mathcal{I}_{n}}\right)^{-1}\left(\left(D_{0}^{\mathcal{I}_{n}}\right)^{\top} W^{\mathcal{I}_{n}} S^{\mathcal{I}_{n}} W^{\mathcal{I}_{n}} D_{0}^{\mathcal{I}_{n}}\right)\left(\left(D_{0}^{\mathcal{I}_{n}}\right)^{\top} W^{\mathcal{I}_{n}} D_{0}^{\mathcal{I}_{n}}\right)^{-1}\right) .
$$

Theorem 6.5 Suppose $W_{T}^{\mathcal{I}_{n}} \rightarrow$ a.s. $W^{\mathcal{I}_{n}}=\left(S^{\mathcal{I}_{n}}\right)^{-1}$. Assumptions $1-5$ and $7-10$ imply

$$
\sqrt{T}\left(\hat{\theta}_{T}^{\mathcal{I}_{n}}-\theta_{0}\right) \rightarrow_{\mathrm{d}} N\left(0,\left(\left(D_{0}^{\mathcal{I}_{n}}\right)^{\top}\left(S^{\mathcal{I}_{n}}\right)^{-1}\left(D_{0}^{\mathcal{I}_{n}}\right)\right)^{-1}\right) .
$$

Moreover, this choice of $W^{\mathcal{I}_{n}}$ is efficient given the moment conditions (55).

The extended over-identified estimator reduces to the over-identified estimator considered in Section 2 when there is a single block of data. Section 6.3 gives examples where there are multiple blocks of data.

We now prove a result analogous to Theorem 3.2. That theorem showed that including the data segment for which some data were missing improved efficiency relative to standard GMM. Here we show that including a new data segment improves efficiency relative to the estimator that includes all data but this segment. Without loss of generality, we consider the full over-identified estimator relative to the over-identified estimator defined over the first $n-1$ blocks of data.

Theorem 6.6 Assume $W_{T}^{\mathcal{I}_{n}} \rightarrow\left(S^{\mathcal{I}_{n}}\right)^{-1}$ and $W_{\left(1-\lambda_{n}\right) T}^{\mathcal{I}_{n-1}} \rightarrow\left(S^{\mathcal{I}_{n-1}}\right)^{-1}$. Assumptions 1-5 and 7-9 $^{-1}$ imply $\hat{\theta}_{T}^{\mathcal{I}_{n}}$ is asymptotically more efficient than $\hat{\theta}_{\left(1-\lambda_{n}\right) T}^{\mathcal{I}_{n-1}}$.

Proof It suffices to compare the asymptotic variance of $\sqrt{T} \hat{\theta}_{T}^{\mathcal{I}_{n}}$ with $\sqrt{T} \hat{\theta}_{\left(1-\lambda_{n}\right) T}^{\mathcal{I}_{n-1}}$. By Theorem 6.5 ,

$$
E\left[\left(1-\lambda_{n}\right) T\left(\hat{\theta}_{\left(1-\lambda_{n}\right) T}^{\mathcal{I}_{n-1}}-\theta_{0}\right)\left(\hat{\theta}_{\left(1-\lambda_{n}\right) T}^{\mathcal{I}_{n-1}}-\theta_{0}\right)^{\top}\right]=\left(\left(D_{0}^{\mathcal{I}_{n-1}}\right)^{\top}\left(S^{\mathcal{I}_{n-1}}\right)^{-1}\left(D_{0}^{\mathcal{I}_{n-1}}\right)\right)^{-1}
$$

where

$$
S^{\mathcal{I}_{n-1}}=\left[\begin{array}{cccc}
\frac{1-\lambda_{n}}{\lambda_{1}} S_{\phi_{1}} & 0 & \cdots & 0 \\
0 & \frac{1-\lambda_{n}}{\lambda_{2}} S_{\phi_{2}} & \cdots & 0 \\
0 & 0 & \ddots & 0 \\
0 & 0 & 0 & \frac{1-\lambda_{n}}{\lambda_{n-1}} S_{\phi_{n-1}}
\end{array}\right]
$$


because data segment $\lambda_{j}$ occupies a fraction $\lambda_{k} /\left(1-\lambda_{n}\right)$ of the data segment $\left(1-\lambda_{n}\right) T$ (note that $\lambda_{j}<1-\lambda_{n}$ because $\left.\sum_{j=1}^{n-1} \lambda_{j}=1-\lambda_{n}\right)$. Therefore,

$$
\begin{aligned}
& E\left[T\left(\hat{\theta}_{\left(1-\lambda_{n}\right) T}^{\mathcal{I}_{n-1}}-\theta_{0}\right)\left(\hat{\theta}_{\left(1-\lambda_{n}\right) T}^{\mathcal{I}_{n-1}}-\theta_{0}\right)^{\top}\right]=\frac{1}{1-\lambda_{n}}\left(\left(D_{0}^{\mathcal{I}_{n-1}}\right)^{\top}\left(S^{\mathcal{I}_{n-1}}\right)^{-1}\left(D_{0}^{\mathcal{I}_{n-1}}\right)\right)^{-1} \\
& =\left(\left(D_{0}^{\mathcal{I}_{n-1}}\right)^{\top}\left[\begin{array}{cccc}
\frac{1}{\lambda_{1}} S_{\phi_{1}} & 0 & \cdots & 0 \\
0 & \frac{1}{\lambda_{2}} S_{\phi_{2}} & \cdots & 0 \\
0 & 0 & \ddots & 0 \\
0 & 0 & 0 & \frac{1}{\lambda_{n-1}} S_{\phi_{n-1}}
\end{array}\right]^{-1}\left(D_{0}^{\mathcal{I}_{n-1}}\right)\right)^{-1} .
\end{aligned}
$$

By (59) and Lemma A.1, it suffices to show that

$$
\left(D_{0}^{\mathcal{I}_{n}}\right)^{\top}\left(S^{\mathcal{I}_{n}}\right)^{-1}\left(D_{0}^{\mathcal{I}_{n}}\right)-\frac{1}{1-\lambda_{n}}\left(D_{0}^{\mathcal{I}_{n-1}}\right)^{\top}\left(S^{\mathcal{I}_{n-1}}\right)^{-1}\left(D_{0}^{\mathcal{I}_{n-1}}\right)
$$

is positive semi-definite. Applying (59), we have

$$
S^{\mathcal{I}_{n}}=\left[\begin{array}{cc}
\frac{1}{1-\lambda_{n}} S^{\mathcal{I}_{n-1}} & 0 \\
0 & \frac{1}{\lambda_{n}} S_{\phi_{n}}
\end{array}\right]
$$

and

$$
D^{\mathcal{I}_{n}}=\left[\left(D^{\mathcal{I}_{n-1}}\right)^{\top} D_{0, \phi_{n}}^{\top}\right]^{\top}
$$

Therefore

$$
\left(D_{0}^{\mathcal{I}_{n}}\right)^{\top}\left(S^{\mathcal{I}_{n}}\right)^{-1}\left(D_{0}^{\mathcal{I}_{n}}\right)-\left(1-\lambda_{n}\right)\left(D_{0}^{\mathcal{I}_{n-1}}\right)^{\top}\left(S^{\mathcal{I}_{n-1}}\right)^{-1}\left(D_{0}^{\mathcal{I}_{n-1}}\right)=\lambda_{n} D_{0, \phi_{n}}^{\top} S_{\phi_{n}}^{-1} D_{0, \phi_{n}},
$$

which is positive semi-definite.

\subsection{Extending the Adjusted-Moment Estimator}

This section shows that the adjusted-moment estimator can also be extended to the case where

there is are series of $n$ lengths, where $n$ is greater than 2. In fact, it is possible to define an adjusted-moment estimator that is asymptotically equivalent to the over-identified estimator, just as in the case where there were two blocks of data. Rather than a formulation (55), the extended adjusted-moment estimator is defined by induction.

An advantage of the adjusted moment estimator over the over-identified estimator was described in Section 3. When the model is exactly identified, and there is a set of series that have data throughout the sample period that depend on a subset of the parameters, the adjusted-moment estimator gives the same estimate for those parameters as simply using the long sample. 
Consider the same set-up as in Section 6.1. To simplify notation, we consider a slightly less general problem than in Section 6.1. We require that all series have a segment in common, in other words, $\pi_{1}=l$ and $\phi_{1}=\{1, \ldots, l\}$. Of course, this segment could be a small portion of the total data available. To inductively define the adjusted-moment estimator, we first give a definition of the adjusted-moment estimator when all series are observed for all the data. This is standard GMM. Then we assume that the adjusted-moment estimator has been defined over the first $n-1$ intervals of data, and extend the adjusted moment estimator to all $n$ intervals of data. This procedure can be used to construct the adjusted-moment estimator over the same patterns of missing data as for the over-identified estimator (assuming that all data has been observed over at least one segment), provided that one starts the construction with the segment over which all the data has been observed (of length $\lambda_{1} T$ ), and then proceeds to the segment where $\pi_{2}<\pi_{1}=l$ moment conditions have been observed, and so forth. While the induction approach may appear somewhat cumbersome, the procedure is quite straightforward, as demonstrated by the examples in Section 6.3.

We begin by defining the adjusted-moment estimator when there is a single data segment and no missing data. This is the standard GMM estimator:

$$
h_{\lambda_{1} T}^{\mathcal{A}_{1}}=g_{\lambda_{1} T}
$$

As in the previous section, the subscript on $h$ denotes the data region over which $h$ is measured. The superscript refers to the fact that it is the adjusted-moment estimator, while the subscript on $\mathcal{A}$ refers to the number of blocks of data. It follows from standard arguments that

$$
E\left[h_{\lambda_{1} T}^{\mathcal{A}_{1}}\left(\theta_{0}\right)\right]=E\left[f\left(x_{t}, \theta_{0}\right)\right]=0
$$

and that

$$
h_{\lambda_{1} T}^{\mathcal{A}_{1}}(\theta) \rightarrow{ }_{\text {a.s. }} E\left[f\left(x_{t}, \theta\right)\right]
$$

as $T \rightarrow \infty$. Assume by induction that

$$
h_{\left(1-\lambda_{n}\right) T}^{\mathcal{A}_{n-1}}(\theta) \rightarrow \text { a.s. } E\left[f\left(x_{t}, \theta\right)\right]
$$

and that

$$
E\left[\left(1-\lambda_{n}\right) T\left(h_{\left(1-\lambda_{n}\right) T}^{\mathcal{A}_{n-1}}\left(\theta_{0}\right)\right)\left(h_{\left(1-\lambda_{n}\right) T}^{\mathcal{A}_{n-1}}\left(\theta_{0}\right)\right)^{\top}\right] \rightarrow_{\text {a.s. }} S^{\mathcal{A}_{n-1}}
$$

for some symmetric, positive-definite matrix $S^{\mathcal{A}_{n-1}}$. Finally, assume that $h_{\left(1-\lambda_{n}\right) T}^{\mathcal{A}_{n-1}}\left(\theta_{0}\right)$ is a linear combination of $g_{\phi_{1}}\left(\theta_{0}\right), \ldots, g_{\phi_{n-1}}\left(\theta_{0}\right)$ with non-random coefficients that do not depend on $T$. That 
is

$$
h_{\left(1-\lambda_{n}\right) T}^{\mathcal{A}_{n-1}}\left(\theta_{0}\right)=M_{n-1}\left[g_{\phi_{1}}\left(\theta_{0}\right)^{\top}, \ldots, g_{\phi_{n-1}}\left(\theta_{0}\right)^{\top}\right]^{\top} .
$$

This allows Theorem 6.1 to be applied. Note that (62) implies that there is a one-to-one correspondence between moment conditions in the adjusted-moment estimator, and moment conditions $f_{i}$.

Let $h_{\phi_{n},\left(1-\lambda_{n}\right) T}^{\mathcal{A}_{n-1}}(\theta)$ denote the $\pi_{n}$ components of $h_{\left(1-\lambda_{n}\right) T}^{\mathcal{A}_{n-1}}(\theta)$ that converge to $E\left[f_{\phi_{n}}\left(x_{t}, \theta\right)\right]$. These are the elements of $h_{\phi_{n},\left(1-\lambda_{n}\right) T}^{\mathcal{A}_{n-1}}(\theta)$ corresponding to moments observed over the new data length. We define the adjusted-moment estimator for $n$ intervals as the residual from a regression of the previous adjusted-moment estimator on the difference between the components of the previous adjusted-moment estimator for which the new data is available, and the sample average over the new segment of data. Define

$$
\begin{aligned}
B^{\mathcal{A}_{n-1}} & =\lim _{T \rightarrow \infty} E\left[T h_{\left(1-\lambda_{n}\right) T}^{\mathcal{A}_{n-1}}\left(\theta_{0}\right)\left(h_{\phi_{n},\left(1-\lambda_{n}\right) T}^{\mathcal{A}_{n-1}}\left(\theta_{0}\right)-g_{\phi_{n},\left(1-\lambda_{n}\right) T}\left(\theta_{0}\right)\right)^{\top}\right] \times \\
& E\left[T\left(h_{\phi_{n},\left(1-\lambda_{n}\right) T}^{\mathcal{A}_{n-1}}\left(\theta_{0}\right)-g_{\phi_{n},\left(1-\lambda_{n}\right) T}\left(\theta_{0}\right)\right)\left(h_{\phi_{n},\left(1-\lambda_{n}\right) T}^{\mathcal{A}_{n-1}}\left(\theta_{0}\right)-g_{\phi_{n},\left(1-\lambda_{n}\right) T}\left(\theta_{0}\right)\right)^{\top}\right]^{-1} .
\end{aligned}
$$

$B^{\mathcal{A}_{n-1}}$ is the $l \times \pi_{n}$ matrix of asymptotic regression coefficients from a regression of the $(n-1)$ st adjusted-moment estimator on $h_{\phi_{n},\left(1-\lambda_{n}\right) T}^{\mathcal{A}_{n-1}}\left(\theta_{0}\right)-g_{\phi_{n}, \lambda_{n} T}\left(\theta_{0}\right)$, appropriately scaled by the square root of the sample length. In practice, $B^{\mathcal{A}_{j}}$ can be replaced by a sample estimate $\hat{B}_{T}^{\mathcal{A}_{j}}$ such that $\hat{B}_{T}^{\mathcal{A}_{j}} \rightarrow$ a.s. $B^{\mathcal{A}_{j}}$ as $T \rightarrow \infty .{ }^{12}$ Finally define the $n$th adjusted-moment estimator as

$$
h_{T}^{\mathcal{A}_{n}}(\theta)=h_{\left(1-\lambda_{n}\right) T}^{\mathcal{A}_{n-1}}(\theta)-B^{\mathcal{A}_{n-1}}\left(h_{\phi_{n},\left(1-\lambda_{n}\right) T}^{\mathcal{A}_{n-1}}(\theta)-g_{\phi_{n}, \lambda_{n} T}(\theta)\right)
$$

When evaluated at $\theta_{0}, h_{T}^{\mathcal{A}_{n}}$ is a regression residual. This completes the definition of the adjustedmoment estimator.

We now verify that the induction hypotheses (62)-(64) are valid for $n$. These are necessary to define (66). ${ }^{13}$ Because

$$
h_{\phi_{n},\left(1-\lambda_{n}\right) T}^{\mathcal{A}_{n-1}}(\theta)-g_{\phi_{n}, \lambda_{n} T}(\theta) \rightarrow{ }_{\text {a.s. }} E\left[f_{\phi_{n}}\left(x_{t}, \theta\right)\right]-E\left[f_{\phi_{n}}\left(x_{t}, \theta\right)\right]=0
$$

\footnotetext{
${ }^{12}$ In that case, (64) would be replaced by the requirement that

$$
h_{\left(1-\lambda_{n}\right) T}^{\mathcal{A}_{n-1}}\left(\theta_{0}\right)=M_{n-1, T}\left[g_{\phi_{1}}\left(\theta_{0}\right)^{\top}, \ldots, g_{\phi_{n-1}}\left(\theta_{0}\right)^{\top}\right]^{\top}
$$

where $\lim _{T} M_{n-1, T} \rightarrow$ a.s. $M_{n-1}$. None of the arguments would change.

${ }^{13}$ Equation (62) insures a one-to-one correspondence between moment conditions and components of $h^{\mathcal{A}_{n}}$. Equation (64) insures that $\sqrt{T} h_{\left(1-\lambda_{n}\right) T}^{\mathcal{A}_{n-1}}$ and $\sqrt{T} g_{\phi_{n},\left(1-\lambda_{n}\right)}$ have an asymptotic distribution that is well-defined (by Theorem 6.1). This implies that $B^{\mathcal{A}_{n-1}}$ is well-defined. Equation (63) will be useful later in determining the asymptotic distribution of the adjusted-moment estimator.
} 
(62) is satisfied for $n$. To show (63) for $n$, note first that Theorem 6.1 implies

$$
\lim _{T \rightarrow \infty} E\left[T g_{\phi_{n}, \lambda_{n} T}\left(\theta_{0}\right) h_{\phi_{n},(1-\lambda) T}^{\mathcal{A}_{n-1}}\left(\theta_{0}\right)^{\top}\right]=0 .
$$

Because $h_{T}^{\mathcal{A}_{n}}\left(\theta_{0}\right)$ is a regression residual, (66) implies

$$
\lim _{T \rightarrow \infty} E\left[T h_{T}^{\mathcal{A}_{n}}\left(\theta_{0}\right) h_{T}^{\mathcal{A}_{n}}\left(\theta_{0}\right)^{\top}\right]=\frac{1}{\left(1-\lambda_{n}\right)} S^{\mathcal{A}_{n-1}}-B^{\mathcal{A}_{n-1}}\left[\frac{1}{\lambda_{n}} S_{\phi_{n}}+\frac{1}{\left(1-\lambda_{n}\right)} S_{\phi_{n}}^{\mathcal{A}_{n-1}}\right]\left(B^{\mathcal{A}_{n-1}}\right)^{\top},
$$

where

$$
S_{\phi_{n}}^{\mathcal{A}_{n-1}}=\lim _{T \rightarrow \infty} E\left[T h_{\phi_{n},\left(1-\lambda_{n}\right) T}^{\mathcal{A}_{n-1}}\left(\theta_{0}\right) h_{\phi_{n},\left(1-\lambda_{n}\right) T}^{\mathcal{A}_{n-1}}\left(\theta_{0}\right)^{\top}\right] .
$$

Clearly (67) is well-defined and symmetric. It is positive definite because $\phi_{n}$ is a strict subset of $\{1, \ldots, l\}$, so not all the variance in $h_{\left(1-\lambda_{n}\right) T}^{\mathcal{A}_{n-1}}$ can be explained by $h_{\phi_{n},\left(1-\lambda_{n}\right) T}^{\mathcal{A}_{n-1}}$. Finally, (64) follows from the form of (66).

It may not be immediately clear that this estimator reduces to the one defined in Section 2 when there are only two blocks of data. In fact, it does reduce to the previously-defined adjustedmoment estimator. As stated in Section $6.1, \lambda_{1}=\lambda$ and $\lambda_{2}=(1-\lambda)$. We also have $\phi_{1}=\{1,2\}$, and $\phi_{2}=\{1\}$. The moment conditions for the first adjusted-moment estimator are the same as in standard GMM:

$$
h_{\lambda_{1} T}^{\mathcal{A}_{1}}=g_{\lambda T}
$$

It follows from (66) that

$$
\begin{aligned}
h_{T}^{\mathcal{A}_{2}} & =h_{\lambda_{1} T}^{\mathcal{A}_{1}}-B^{\mathcal{A}_{1}}\left(h_{1, \lambda_{1} T}^{\mathcal{A}_{1}}-g_{\phi_{2}, \lambda_{2} T}\right) \\
& =g_{\lambda T}-B^{\mathcal{A}_{1}}\left(g_{1, \lambda T}-g_{1,(1-\lambda) T}\right) .
\end{aligned}
$$

By (65),

$$
\begin{aligned}
B^{\mathcal{A}_{1}} & =\lim _{T \rightarrow \infty} E\left[T g_{\lambda T}\left(g_{1, \lambda T}-g_{1,(1-\lambda) T}\right)^{\top}\right]\left(E\left[T\left(g_{1, \lambda T}-g_{1,(1-\lambda) T}\right)\left(g_{1, \lambda T}-g_{1,(1-\lambda) T}\right)^{\top}\right]\right)^{-1} \\
& =\frac{1}{\lambda}\left(\begin{array}{c}
S_{11} \\
S_{21}
\end{array}\right)\left[\left(\frac{1}{1-\lambda}+\frac{1}{\lambda}\right) S_{11}\right]^{-1} \\
& =\left(\begin{array}{c}
(1-\lambda) I \\
(1-\lambda) B_{21}
\end{array}\right)
\end{aligned}
$$

where we have suppressed the argument $\theta_{0}$ in the first line. Therefore, the moment conditions for the adjusted-moment estimator equal

$$
h_{T}^{\mathcal{A}_{2}}=\left(\begin{array}{l}
g_{1, \lambda T}+(1-\lambda)\left(g_{1,(1-\lambda) T}-g_{1, \lambda T}\right) \\
g_{2, \lambda T}+(1-\lambda) B_{21}\left(g_{1,(1-\lambda) T}-g_{1, \lambda T}\right)
\end{array}\right),
$$


which are the same moment conditions as those given in Section $2 .{ }^{14}$

The usual asymptotic results hold for the extended adjusted-moment estimator. The following lemma is helpful:

Lemma 6.1 Assumptions 1-5 imply

$$
\sqrt{T}\left(\begin{array}{c}
h_{\left(1-\lambda_{n}\right) T}^{\mathcal{A}_{n-1}}\left(\theta_{0}\right) \\
g_{\phi_{n}, \lambda_{n} T}\left(\theta_{0}\right)
\end{array}\right) \rightarrow_{\mathrm{d}} N\left(0,\left[\begin{array}{cc}
\frac{1}{1-\lambda_{n}} S^{\mathcal{A}_{n-1}} & 0 \\
0 & \frac{1}{\lambda_{n}} S_{\phi_{n}}
\end{array}\right]\right) .
$$

Proof It follows from (64) and Theorem 6.1 that $\sqrt{T} h_{\left(1-\lambda_{n}\right) T}^{\mathcal{A}_{n-1}}\left(\theta_{0}\right)$ and $\sqrt{T} g_{\phi_{n}, \lambda_{n} T}\left(\theta_{0}\right)$ are asymptotically independent, and that each are asymptotically normally distributed. The form of the asymptotic variance follows from (63) and Theorem 6.1 .

This lemma implies that the sample moment conditions for the $n$th adjusted-moment estimator, scaled by $\sqrt{T}$ are asymptotically normally distributed. Equation (66) implies an inductive equation for the variance.

Theorem 6.7 Assumptions 1-5 imply

$$
\sqrt{T} h_{T}^{\mathcal{A}_{n}}\left(\theta_{0}\right) \rightarrow_{\mathrm{d}} N\left(0, S^{\mathcal{A}_{n}}\right)
$$

where $S^{\mathcal{A}_{n}}$ is defined inductively as

$$
S^{\mathcal{A}_{n}}=\frac{1}{1-\lambda_{n}} S^{\mathcal{A}_{n-1}}-B^{\mathcal{A}_{n-1}}\left[\frac{1}{\lambda_{n}} S_{\phi_{n}}+\frac{1}{1-\lambda_{n}} S_{\phi_{n}}^{\mathcal{A}_{n-1}}\right]\left(B^{\mathcal{A}_{n-1}}\right)^{\top}
$$

with

$$
S^{\mathcal{A}_{1}}=S
$$

As with the extended over-identified estimator, any positive definite weighting matrix can be used with moment conditions $h_{T}^{\mathcal{A}_{n}}$ to produce a consistent estimator. As usual, we will emphasize the case when the weighting matrix converges almost surely to $S^{\mathcal{A}_{n}}$. Define

$$
\hat{\theta}_{T}^{\mathcal{A}_{n}}=\operatorname{argmin}_{\theta} h_{T}^{\mathcal{A}_{n}}(\theta)^{\top} W_{T}^{\mathcal{A}_{n}} h_{T}^{\mathcal{A}_{n}}(\theta),
$$

where $W_{T}^{\mathcal{A}_{n}}$ satisfies Assumption 11:

Assumption 11 The weighting matrix $W_{T}^{\mathcal{A}_{n}}$ converges almost surely to a positive-definite matrix $W^{\mathcal{A}_{n}}$.

\footnotetext{
${ }^{14}$ Here we make use of the equation $g_{1 T}=\lambda g_{1, \lambda_{1} T}+(1-\lambda) g_{1,(1-\lambda) T}$.
} 
Consistency for the extended adjusted moment estimator follows from the fact that

$$
h_{T}^{\mathcal{A}_{n}}(\theta) \rightarrow \text { a.s. } E\left[f\left(x_{t}, \theta\right)\right]
$$

(proved above by induction) and the arguments of Section 2.

Theorem 6.8 Assumptions $1-5$, and 11 imply that as $T \rightarrow \infty, \hat{\theta}^{\mathcal{A}_{n}} \rightarrow$ a.s. $\theta_{0}$.

Similarly, it is possible to show that the estimator is asymptotically normally distributed:

Theorem 6.9 Assumptions 1-5, 7-9 and 11 imply that as $T \rightarrow \infty$,

$$
\sqrt{T}\left(\hat{\theta}_{T}^{\mathcal{A}_{n}}-\theta_{0}\right) \rightarrow_{\mathrm{d}} N\left(0,\left(D_{0}^{\top} W^{\mathcal{A}_{n}} D_{0}\right)^{-1}\left(D_{0}^{\top} W^{\mathcal{A}_{n}} S^{\mathcal{A}_{n}} W^{\mathcal{A}_{n}} D_{0}\right)\left(D_{0}^{\top} W^{\mathcal{A}_{n}} D_{0}\right)^{-1}\right) .
$$

Proof We show by induction on $n$ that

$$
\frac{\partial h_{T}^{\mathcal{A}_{n}}}{\partial \theta}\left(\theta_{0}\right) \rightarrow_{\text {a.s. }} E\left[\left.\frac{\partial f}{\partial \theta}\left(x_{t}, \theta\right)\right|_{\theta_{0}}\right] \equiv D_{0} .
$$

By definition, and White and Domowitz (1984, Theorem 2.3) it follows that

$$
\frac{\partial h_{\lambda_{1} T}^{\mathcal{A}_{1}}}{\partial \theta}\left(\theta_{0}\right)=\frac{\partial g_{\lambda_{1} T}}{\partial \theta}\left(\theta_{0}\right) \rightarrow \text { a.s. } D_{0} .
$$

Assume (72) holds for $n-1$. By (66),

$$
h_{T}^{\mathcal{A}_{n}}(\theta)=h_{\left(1-\lambda_{n}\right) T}^{\mathcal{A}_{n-1}}(\theta)-B^{\mathcal{A}_{n-1}}\left(h_{\phi_{n},\left(1-\lambda_{n}\right) T}^{\mathcal{A}_{n-1}}(\theta)-g_{\phi_{n}, \lambda_{n} T}(\theta)\right) .
$$

Applying White and Domowitz (1984, Theorem 2.3) again, it follows that

$$
\frac{\partial g_{\phi_{n}, \lambda_{n} T}}{\partial \theta}(\theta) \rightarrow \text { a.s. } D_{0} .
$$

Taking limits on both sides of (73) and using the induction hypothesis produces the desired result. The rest of the proof follows along the same lines as that of Theorem 2.3 in Section 2.

Lastly, given moments $h_{T}^{\mathcal{A}_{n}}$, the most efficient asymptotic weighting matrix is the inverse of the variance of these moments.

Theorem 6.10 Suppose $W_{T}^{\mathcal{A}_{n}} \rightarrow$ a.s. $W^{\mathcal{A}_{n}}=\left(S^{\mathcal{A}_{n}}\right)^{-1}$. Assumptions 1-5 and 7-9 imply that

$$
\sqrt{T}\left(\hat{\theta}_{T}^{\mathcal{A}_{n}}-\theta_{0}\right) \stackrel{a}{\sim} N\left(0,\left(D_{0}^{\top}\left(S^{\mathcal{A}_{n}}\right)^{-1} D_{0}\right)^{-1}\right) .
$$

Moreover, this choice of $W^{\mathcal{A}_{n}}$ is efficient given the moment conditions (55). 
While the extended adjusted-moment estimator appears completely different from the extended over-identified estimator, they are asymptotically equivalent.

Theorem 6.11 Assume that $W_{T}^{\mathcal{A}_{n}} \rightarrow\left(S^{\mathcal{A}_{n}}\right)^{-1}$ and $W_{T}^{\mathcal{I}_{n}} \rightarrow\left(S^{\mathcal{I}_{n}}\right)^{-1}$. For any integer $n$, assumptions 1-5 and 7-9 imply that the extended adjusted-moment estimator (71) is asymptotically equivalent to the extended over-identified estimator (57).

A full proof is given in the Appendix. The structure of the proof is similar to that of Theorem 3.1. The preceding theorems show that it suffices to compare the asymptotic variances. Then matrix partition results are used to relate the inverse of the asymptotic variance for the over-identified estimator to the asymptotic variance of the adjusted-moment estimator.

Intuitively, the reason for the equivalence is that both estimators insure that each additional segment reduces the variance in the most efficient way. The variance reduction is easier to see in the case of the over-identified estimator, where each additional segment introduces a new moment condition. The efficient weighting matrix, along with a standard "diversification" argument insures that the new estimator will have a smaller variance than the old estimator. For the adjustedmoment estimator, each step of the further reduces the variance of the moment conditions, because the new moment conditions are defined as regression residuals from the previous step. As regression residuals, they must have smaller variance than the variable on the right-hand side of the regression - the previous moment conditions.

Theorem 6.11 shows that the extended adjusted moment estimator is asymptotically equivalent to the extended over-identified estimator. By Theorem 6.6, we can conclude that adding a block of data always increases efficiency for the adjusted-moment estimator.

Corollary 6.1 Assume that $W_{T}^{\mathcal{A}_{n}} \rightarrow\left(S^{\mathcal{A}_{n}}\right)^{-1}$ and $W_{\left(1-\lambda_{n}\right) T}^{\mathcal{A}_{n-1}} \rightarrow\left(S^{\mathcal{A}_{n-1}}\right)^{-1}$. Assumptions 1-5 and 7-9 imply $\sqrt{T} \hat{\theta}_{T}^{\mathcal{A}_{n}}$ is asymptotically more efficient than $\sqrt{T} \hat{\theta}_{\left(1-\lambda_{n}\right) T}^{\mathcal{A}_{n-1}}$.

Defining the adjusted-moment estimator as a regression residual has some appealing properties; it facilitates the proof of equivalence for the over-identified estimator, and it demonstrates clearly the reduction in variance. In other respects, it may appear counterintuitive. In the next section, we compute three examples of the adjusted-moment estimator and show that indeed, they have an interpretation that is equally appealing as in the case where only one data block is missing.

\subsection{Examples}

This section computes explicit estimators for three examples of missing data patterns. The first example is like that explored in Section 2, except here data is missing at both ends of the sample 
for one of the series, rather than just at the beginning. The second example is where there are three different starting dates, but all data have the same ending dates. This case is treated in Stambaugh (1997) in a maximum-likelihood setting, and applied to international data. This example shows that our methods can be easily applied to this setting as well. Given the form of the estimators for examples 1 and 2, one could easily combine the reasoning and put together an example where the data have both different starting and ending dates, but that the available data are "nested" (e.g. there are three series, the first of which is observed for the full sample, the second is observed for a subset of the dates, and the third is observed for a subset of the dates for which the second is observed). Little and Rubin (2002) refer to this condition as monotonicity, and derive a maximum likelihood estimator under normality and independent, identically distributed observations.

The last example is a case where the series have different starting dates and different ending dates, but that the series that start later also end later. This example illustrates the power of our generalization above, as its form for the adjusted-moment estimator is non-obvious.

In all of these cases, we derive both the over-identified estimator and the adjusted-moment estimator. For the over-identified estimator, we derive both the moment conditions, the optimal matrix, and the form of $D_{0}^{\mathcal{I}}$. For the adjusted-moment matrix, the derivative of the moments always equals $D_{0}$ asymptotically. The optimal weighting matrix is the inverse of the variance of the moments, which can be computed from (69). If the original problem is exactly identified, it will remain so with the adjusted-moment estimator. Also, the extended adjusted-moment estimator will be consistent, and efficient relative to the estimator that uses a shorter length of data, for any choice of positive-definite weighting matrix.

\subsubsection{Data missing at both ends}

The first example is similar to the case in Section 2, except that data from the second set of series is missing not only at the beginning of the sample, but also at the end. ${ }^{15}$ Figure 3 illustrates this pattern of missing data. As in Section 2, group the moment conditions observed for the full data set into a vector $f_{1}\left(x_{1 t}, \theta\right)$, and moment conditions only observed for the middle segment into a vector $f_{2}\left(x_{t}, \theta\right)$. This situation would occur if the series for which data is missing at the start of the sample also is updated less frequently. We use $g_{1, \cdot}(\theta)$ to denote partial sums of $f_{1}\left(x_{1 t}, \theta\right)$ and $g_{2, \cdot}(\theta)$ to denote partial sums of $f_{2}\left(x_{t}, \theta\right)$, where - will represent the length of the segment over which the

\footnotetext{
${ }^{15}$ If the observations were independent, then this case is clearly identical to that described in Section 2 because the data points could be rearranged without effecting the joint distribution. Under dependence, this does not follow immediately.
} 


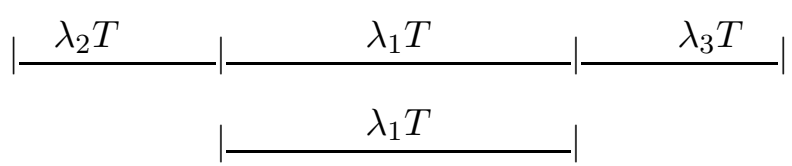

Figure 3: Illustration of Example 1. Example 1 explicitly calculates the extended estimators for data missing at both ends. The notation above the horizontal lines refers to the length of each segment as a function of the sample size $T$.

observation is taken. The notation for sub-matrices of $S$ and $D_{0}$ follows the same conventions as in Section 2.

As shown in Figure 3, $\lambda_{1}$ is the length of the middle segment divided by the total data length. Without loss of generality, we assume $\lambda_{2}$ is the length of the first segment of missing data divided by the total length (we could also have set $\lambda_{2}$ equal to the length of the second segment of missing data divided by the total data length). The moment conditions for the over-identified estimator $h_{T}^{\mathcal{I}_{3}}$ are

$$
h_{T}^{\mathcal{I}_{3}}(\theta)=\left[g_{1, \lambda_{1} T}(\theta)^{\top} g_{2, \lambda_{1} T}(\theta)^{\top} g_{1, \lambda_{2} T}(\theta)^{\top} g_{1, \lambda_{3} T}(\theta)^{\top}\right]^{\top} .
$$

Then the results in Section 6.1 imply that $\sqrt{T} h_{T}^{\mathcal{I}_{3}}(\theta)$ has asymptotic variance ${ }^{16}$

$$
S^{\mathcal{I}_{3}}=\left[\begin{array}{cccc}
\frac{1}{\lambda_{1}} S_{11} & \frac{1}{\lambda_{1}} S_{12} & 0 & 0 \\
\frac{1}{\lambda_{1}} S_{21} & \frac{1}{\lambda_{1}} S_{22} & 0 & 0 \\
0 & 0 & \frac{1}{\lambda_{2}} S_{11} & 0 \\
0 & 0 & 0 & \frac{1}{\lambda_{3}} S_{11}
\end{array}\right]
$$

The extended over-identified estimator with efficient weighting matrix is therefore

$$
\hat{\theta}^{\mathcal{I}_{3}}=\operatorname{argmin}_{\theta} h_{T}^{\mathcal{I}_{3}}(\theta)^{\top} W_{T}^{\mathcal{I}_{3}} h_{T}^{\mathcal{I}_{3}}(\theta), \quad W_{T}^{\mathcal{I}_{3}} \rightarrow_{\text {a.s. }}\left(S^{\mathcal{I}_{3}}\right)^{-1}
$$

for $W_{T}^{\mathcal{I}_{3}}$ positive definite. The asymptotic distribution is given by

$$
\sqrt{T}\left(\hat{\theta}_{T}^{\mathcal{I}_{3}}-\theta_{0}\right) \rightarrow_{\mathrm{d}} N\left(0,\left(\left(D_{0}^{\mathcal{I}_{3}}\right)^{\top}\left(S^{\mathcal{I}_{3}}\right)^{-1}\left(D_{0}^{\mathcal{I}_{3}}\right)\right)^{-1}\right)
$$

where

$$
D^{\mathcal{I}_{3}}=\left[\begin{array}{llll}
D_{0,1}^{\top} & D_{0,2}^{\top} & D_{0,1}^{\top} & D_{0,1}^{\top}
\end{array}\right]^{\top} .
$$

We now describe the extended adjusted-moment estimator. The moment conditions for the first adjusted-moment estimator are the same as in standard GMM:

$$
h_{\lambda_{1} T}^{\mathcal{A}_{1}}=g_{\lambda_{1} T} .
$$

\footnotetext{
${ }^{16}$ The asymptotic variance does not take exactly the same form as $S^{\mathcal{I}}$ in Section 2 . The reason for the discrepancy is that $S^{\mathcal{I}}$ was defined as the asymptotic variance of the moment conditions scaled by $\sqrt{\lambda T}$, while $S^{\mathcal{I}_{3}}$ is the asymptotic variance of the moment conditions scaled by $\sqrt{T}$.
} 
Substituting (75) into (66) yields the adjusted-moment estimator that includes the $\lambda_{2}$ block:

$$
h_{\left(\lambda_{1}+\lambda_{2}\right) T}^{\mathcal{A}_{2}}=g_{\lambda_{1} T}-B^{\mathcal{A}_{1}}\left(g_{1, \lambda_{1} T}-g_{1, \lambda_{2} T}\right),
$$

where

$$
\begin{aligned}
B^{\mathcal{A}_{1}} & =\lim _{T \rightarrow \infty} E\left[\left(\lambda_{1}+\lambda_{2}\right) T g_{\lambda_{1} T}\left(g_{1, \lambda_{1} T}-g_{1, \lambda_{2} T}\right)^{\top}\right]\left(E\left[\left(\lambda_{1}+\lambda_{2}\right) T\left(g_{1, \lambda_{1} T}-g_{1, \lambda_{2} T}\right)\left(g_{1, \lambda_{1} T}-g_{1, \lambda_{2} T}\right)^{\top}\right]\right)^{-1} \\
& =\frac{\lambda_{2}}{\lambda_{1}+\lambda_{2}}\left(\begin{array}{l}
I \\
B_{21}
\end{array}\right)
\end{aligned}
$$

where we have suppressed the $\theta_{0}$ argument in the first line. Substituting into (76) produces

$$
h_{\left(\lambda_{1}+\lambda_{2}\right) T}^{\mathcal{A}_{2}}=\left(\begin{array}{l}
g_{1,\left(\lambda_{1}+\lambda_{2}\right) T} \\
g_{2, \lambda_{1} T}+\frac{\lambda_{2}}{\lambda_{1}+\lambda_{2}} B_{21}\left(g_{1, \lambda_{2} T}-g_{1, \lambda_{1} T}\right)
\end{array}\right)
$$

which is the same estimator described in Section 2, except that the length of the sample is taken to be $\left(\lambda_{1}+\lambda_{2}\right) T$ rather than $T .{ }^{17}$

To construct the full adjusted-moment estimator for this case, we apply (66) again:

$$
h_{T}^{\mathcal{A}_{3}}=h_{\left(\lambda_{1}+\lambda_{2}\right) T}^{\mathcal{A}_{2}}-B^{\mathcal{A}_{2}}\left(h_{1,\left(\lambda_{1}+\lambda_{2}\right) T}^{\mathcal{A}_{2}}-g_{1, \lambda_{3} T}\right),
$$

where

$$
\begin{aligned}
B^{\mathcal{A}_{2}}= & \lim _{T \rightarrow \infty} E\left[T h_{\left(\lambda_{1}+\lambda_{2}\right) T}^{\mathcal{A}_{2}}\left(h_{1,\left(\lambda_{1}+\lambda_{2}\right) T}^{\mathcal{A}_{2}}-g_{1, \lambda_{3} T}\right)^{\top}\right] \\
\times\left(E\left[T\left(h_{\left(\lambda_{1}+\lambda_{2}\right) T}^{\mathcal{A}_{2}}-g_{1, \lambda_{3} T}\right)\left(h_{1,\left(\lambda_{1}+\lambda_{2}\right) T}^{\mathcal{A}_{2}}-g_{1, \lambda_{3} T}\right)^{\top}\right]\right)^{-1} & (80) \\
= & \lim _{T \rightarrow \infty} E\left[T h_{\left(\lambda_{1}+\lambda_{2}\right) T}^{\mathcal{A}_{2}}\left(g_{1,\left(\lambda_{1}+\lambda_{2}\right) T}-g_{1, \lambda_{3} T}\right)^{\top}\right]\left(E\left[T\left(g_{1,\left(\lambda_{1}+\lambda_{2}\right) T}-g_{1, \lambda_{3} T}\right)\left(g_{1,\left(\lambda_{1}+\lambda_{2}\right) T}-g_{1, \lambda_{3} T}\right)^{\top}\right]\right)^{-1}
\end{aligned}
$$

It follows from Theorem 6.1 that

$$
\lim _{T \rightarrow \infty} E\left[T\left(g_{1,\left(\lambda_{1}+\lambda_{2}\right) T}-g_{1, \lambda_{3} T}\right)\left(g_{1,\left(\lambda_{1}+\lambda_{2}\right) T}-g_{1, \lambda_{3} T}\right)^{\top}\right]=\left(\frac{1}{\lambda_{3}}+\frac{1}{\lambda_{1}+\lambda_{2}}\right) S_{11} .
$$

Using (77) and the same argument,

$$
\lim _{T \rightarrow \infty} E\left[T h_{1,\left(\lambda_{1}+\lambda_{2}\right) T}^{\mathcal{A}_{2}}\left(g_{1,\left(\lambda_{1}+\lambda_{2}\right) T}-g_{1, \lambda_{3} T}\right)^{\top}\right]=\frac{1}{\lambda_{1}+\lambda_{2}} S_{11} .
$$

\footnotetext{
${ }^{17}$ Here and in the following computations, we make use of the equation

$$
g_{1,\left(\lambda_{1}+\lambda_{2}\right) T}=\frac{\lambda_{1}}{\lambda_{1}+\lambda_{2}} g_{1, \lambda_{1} T}+\frac{\lambda_{2}}{\lambda_{1}+\lambda_{2}} g_{1, \lambda_{2} T} .
$$
}


Finally, Theorem 6.1 and the same reasoning used to show (21) that

$$
\begin{aligned}
\lim _{T \rightarrow \infty} E\left[T h_{2,\left(\lambda_{1}+\lambda_{2}\right) T}^{\mathcal{A}_{2}}\left(g_{1,\left(\lambda_{1}+\lambda_{2}\right) T}-g_{1, \lambda_{3} T}\right)^{\top}\right] & =\lim _{T \rightarrow \infty} E\left[T h_{2,\left(\lambda_{1}+\lambda_{2}\right) T}^{\mathcal{A}_{2}} g_{1,\left(\lambda_{1}+\lambda_{2}\right) T}^{\top}\right] \\
& =\frac{1}{\lambda_{1}+\lambda_{2}} S_{21}
\end{aligned}
$$

where we have continued to suppressed the argument $\theta_{0}$. Combining (81), (82), and (83), and rearranging,

$$
B^{\mathcal{A}_{2}}=\lambda_{3}\left(\begin{array}{l}
I \\
B_{21}
\end{array}\right)
$$

Substituting into (79) and rearranging produces ${ }^{18}$

$$
h_{T}^{\mathcal{A}_{3}}=\left(\begin{array}{l}
g_{1, T} \\
g_{2, \lambda_{1} T}+\left(\lambda_{2}+\lambda_{3}\right) B_{21}\left(\frac{\lambda_{2}}{\lambda_{2}+\lambda_{3}} g_{1, \lambda_{2} T}+\frac{\lambda_{3}}{\lambda_{2}+\lambda_{3}} g_{1, \lambda_{3} T}-g_{1, \lambda_{1} T}\right)
\end{array}\right) .
$$

Several features of this extended adjusted-moment estimator are worth noting. First, the moment condition for the series observed for the full data set is the same as if these series were estimated independently of the second set of moments. The basic adjusted-moment estimator described in Section 2) also had this property, and, as we argued in Section 3 this may be a reason to prefer the adjusted-moment estimator over the over-identified estimator. Second, the adjustment to the moments of the second series is the same as if the intervals $\lambda_{2}$ and $\lambda_{3}$ were contiguous rather than separated by $\lambda_{1}$. For our asymptotic results, it does not matter whether the blocks defined by starting and ending points are contiguous.

\subsubsection{Data missing in a monotonic pattern}

The second example represents a problem dealt with in detail in a maximum likelihood context by Little and Rubin (2002) and Stambaugh (1997). Here, the data series all end at the same point, but may start from more than two different points. This may occur, for example, if one is using international data as in the study by Stambaugh. Figure 4 illustrates the missing data pattern in this case. For ease of notation, we illustrate the extended over-identified and extended adjustedmoment estimators for the case where there are three starting dates. Extending the method further to more than three starting dates is straightforward.

\footnotetext{
${ }^{18}$ Here and in the following example, we use the fact that $\lambda_{1}+\lambda_{2}+\lambda_{3}=1$, and that

$$
\begin{aligned}
g_{1, T} & =\left(\lambda_{1}+\lambda_{2}\right) g_{1,\left(\lambda_{1}+\lambda_{2}\right) T}+\lambda_{3} g_{2, \lambda_{3} T} \\
& =\lambda_{1} g_{1, \lambda_{1} T}+\lambda_{2} g_{1, \lambda_{2} T}+\lambda_{3} g_{2, \lambda_{3} T} .
\end{aligned}
$$
}




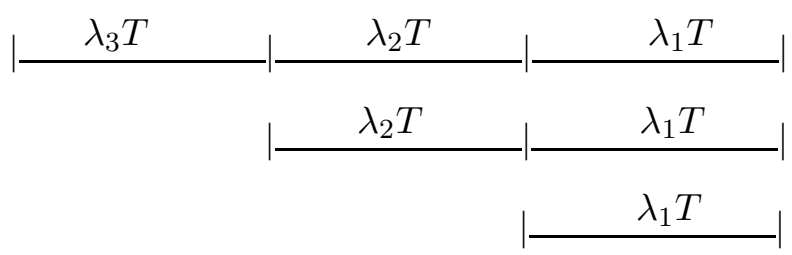

Figure 4: Illustration of Example 2. Example 2 explicitly calculates the extended estimators for data missing in a monotonic pattern. The notation above the horizontal lines refers to the length of each segment as a function of the sample size $T$.

As shown in Figure $4, \lambda_{1}$ is the length of the final segment divided by the total data length. This is because all series are observed for the segment of length $\lambda_{1} T$. A subset of these series are also observed for the middle segment: this has length $\lambda_{2} T$. A smaller subset is also observed for the first segment, of length $\lambda_{3} T=\left(1-\lambda_{1}-\lambda_{2}\right) T$. Following the notational convention of Section 2 and the previous example, we group the moment conditions observed for the full data set into a vector $f_{1}\left(x_{1 t}, \theta\right)$, the moment conditions observed for the last two data intervals into a vector $f_{2}\left(x_{1 t}, x_{2 t}, \theta\right)$, and the moment conditions observed only for the last data segment into a vector $f_{3}\left(x_{1 t}, x_{2 t}, x_{3 t}, \theta\right)$. The notation for sub-vectors of $g$ and submatrices of $D_{0}$ and $S$ follows the same convention as in the previous example.

The moment conditions for the over-identified estimator $h_{T}^{\mathcal{I}_{3}}$ are

$$
h_{T}^{\mathcal{I}_{3}}(\theta)=\left[g_{1, \lambda_{1} T}(\theta)^{\top} g_{2, \lambda_{1} T}(\theta)^{\top} g_{3, \lambda_{1} T}(\theta)^{\top} g_{1, \lambda_{2} T}(\theta)^{\top} g_{2, \lambda_{2} T}(\theta)^{\top} g_{1, \lambda_{3} T}(\theta)^{\top}\right] .
$$

The results of Section 6.1 imply that $\sqrt{T} h_{T}^{\mathcal{I}_{3}}$ has asymptotic variance

$$
S^{\mathcal{I}_{3}}=\left[\begin{array}{cccccc}
\frac{1}{\lambda_{1}} S_{11} & \frac{1}{\lambda_{1}} S_{12} & \frac{1}{\lambda_{1}} S_{13} & 0 & 0 & 0 \\
\frac{1}{\lambda_{1}} S_{21} & \frac{1}{\lambda_{1}} S_{22} & \frac{1}{\lambda_{1}} S_{23} & 0 & 0 & 0 \\
\frac{1}{\lambda_{1}} S_{31} & \frac{1}{\lambda_{1}} S_{32} & \frac{1}{\lambda_{1}} S_{33} & 0 & 0 & 0 \\
0 & 0 & 0 & \frac{1}{\lambda_{2}} S_{11} & \frac{1}{\lambda_{2}} S_{12} & 0 \\
0 & 0 & 0 & \frac{1}{\lambda_{2}} S_{21} & \frac{1}{\lambda_{2}} S_{22} & 0 \\
0 & 0 & 0 & 0 & 0 & \frac{1}{\lambda_{3}} S_{11}
\end{array}\right] .
$$

In this example the extended over-identified estimator is therefore

$$
\hat{\theta}_{T}^{\mathcal{I}_{3}}=\operatorname{argmin}_{\theta} h_{T}^{\mathcal{I}_{3}}(\theta)^{\top} W_{T}^{\mathcal{I}_{3}} h_{T}^{\mathcal{I}_{3}}(\theta),
$$

for $W_{T}^{\mathcal{I}_{3}}$ positive definite and $W_{T}^{\mathcal{I}_{3}} \rightarrow$ a.s. $\left(S^{\mathcal{I}_{3}}\right)^{-1}$. The estimator has asymptotic distribution

$$
\sqrt{T}\left(\hat{\theta}_{T}^{\mathcal{I}_{3}}-\theta_{0}\right) \rightarrow_{\mathrm{d}} N\left(0,\left(\left(D_{0}^{\mathcal{I}_{3}}\right)^{\top}\left(S^{\mathcal{I}_{3}}\right)^{-1}\left(D_{0}^{\mathcal{I}_{3}}\right)\right)^{-1}\right)
$$


where

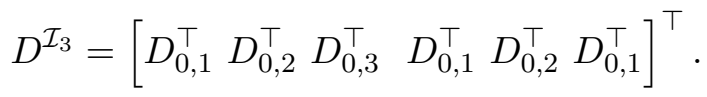

We now describe the extended adjusted-moment estimator. The first step is the same as standard GMM for the three series:

$$
h_{\lambda_{1} T}^{\mathcal{A}_{1}}=\left[\begin{array}{c}
g_{1, \lambda_{1} T} \\
g_{2, \lambda_{1} T} \\
g_{3, \lambda_{1} T}
\end{array}\right] .
$$

The second step is the same as the second step in the example above. However, here two sets of series are observed for the longer sample, $g_{1}$ and $g_{2}$. Therefore

$$
B^{\mathcal{A}_{1}}=\frac{\lambda_{2}}{\lambda_{1}+\lambda_{2}}\left(\begin{array}{l}
I \\
B_{3 \cdot 12}
\end{array}\right)
$$

and

$$
h_{\left(\lambda_{1}+\lambda_{2}\right) T}^{\mathcal{A}_{2}}=\left[\begin{array}{l}
g_{1,\left(\lambda_{1}+\lambda_{2}\right) T} \\
g_{2,\left(\lambda_{1}+\lambda_{2}\right) T} \\
g_{3, \lambda_{1} T}+\frac{\lambda_{2}}{\lambda_{1}+\lambda_{2}} B_{3 \cdot 12}\left(\begin{array}{c}
g_{1, \lambda_{2} T}-g_{1, \lambda_{1} T} \\
g_{2, \lambda_{2} T}-g_{2, \lambda_{1} T}
\end{array}\right)
\end{array}\right],
$$

where $B_{3.12}$ are the coefficients from a multivariate regression on the third set of series on the first two:

$$
B_{3 \cdot 12}=\left[\begin{array}{ll}
S_{31} & S_{32}
\end{array}\right]\left[\begin{array}{ll}
S_{11} & S_{12} \\
S_{21} & S_{22}
\end{array}\right]^{-1}
$$

In the third step, we add the segment of length $\lambda_{3} T$. Then

$$
h_{T}^{\mathcal{A}_{3}}=h_{\left(\lambda_{1}+\lambda_{2}\right) T}^{\mathcal{A}_{2}}-B^{\mathcal{A}_{2}}\left(h_{1,\left(\lambda_{1}+\lambda_{2}\right) T}^{\mathcal{A}_{2}}-g_{1, \lambda_{3} T}\right),
$$

where the expression for $B^{\mathcal{A}_{2}}$ is given by (80). It follows from Theorem 6.1 that

$$
\lim _{T \rightarrow \infty} E\left[T\left(h_{1,\left(\lambda_{1}+\lambda_{2}\right) T}^{\mathcal{A}_{2}}-g_{1, \lambda_{3} T}\right)\left(h_{1,\left(\lambda_{1}+\lambda_{2}\right) T}^{\mathcal{A}_{2}}-g_{1, \lambda_{3} T}\right)^{\top}\right]^{-1}=\left(\frac{1}{\lambda_{3}}+\frac{1}{\lambda_{1}+\lambda_{2}}\right) S_{11} .
$$

Similar reasoning shows that

$$
\lim _{T \rightarrow \infty} E\left[T h_{j,\left(\lambda_{1}+\lambda_{2}\right) T}^{\mathcal{A}_{2}}\left(h_{1,\left(\lambda_{1}+\lambda_{2}\right) T}^{\mathcal{A}_{2}}-g_{1, \lambda_{3} T}\right)^{\top}\right]=\frac{1}{\lambda_{1}+\lambda_{2}} S_{j 1}, \quad j=1,2,3,
$$

where we have made use of (21) for $j=3$. Substituting into (85), and applying footnote 18 results in

$$
h_{T}^{\mathcal{A}_{3}}=\left[\begin{array}{l}
g_{1 T} \\
g_{2,\left(\lambda_{1}+\lambda_{2}\right) T}+\lambda_{3} B_{21}\left(g_{1, \lambda_{3} T}-g_{1,\left(\lambda_{1}+\lambda_{2}\right) T}\right) \\
g_{3, \lambda_{1} T}+\frac{\lambda_{2}}{\lambda_{1}+\lambda_{2}} B_{3 \cdot 12}\left(\begin{array}{c}
g_{1, \lambda_{2} T}-g_{1, \lambda_{1} T} \\
g_{2, \lambda_{2} T}-g_{2, \lambda_{1} T}
\end{array}\right)+\lambda_{3} B_{31}\left(g_{1, \lambda_{3} T}-g_{1,\left(\lambda_{1}+\lambda_{2}\right) T}\right)
\end{array}\right],
$$

where $B_{31}=S_{31} S_{11}^{-1}$. 
Note that the moment conditions for the data series observed for the full data set are the same as if these series were estimated independently of the second and third set of moments. Indeed, the moment conditions for the data series observed for both $\lambda_{1}$ and $\lambda_{2}$ are the same as if these series were estimated (using the adjusted-moment estimator) without the third set of moments. Thus the principle advantage of the adjusted-moment estimator for two starting dates is retained and extended in this example with multiple starting dates.

In constructing this estimator, we have assumed that all the missing data occurs at the beginning of the sample. However, the estimator would take the same form if the missing data were at the end. Indeed, as the previous section shows, it suffices to have the data observed for the third set of series be nested in the data observed for the second set, which is nested in the data observed for the first set. In other words, data could be missing at both ends of the sample. In this case, the adjusted-moment estimator would take the same form as above.

\subsubsection{Data missing in a non-monotonic pattern}

Our last example represents a case not handled in the maximum likelihood settings of Little and Rubin (2002) and Stambaugh (1997). In this example, there are two sets of moments. These moments have different starting dates and different ending dates, as in the first example. However, the series that ends earlier also starts earlier, so neither series is observed for the full length. Figure 5 illustrates the pattern of missing data in this example.

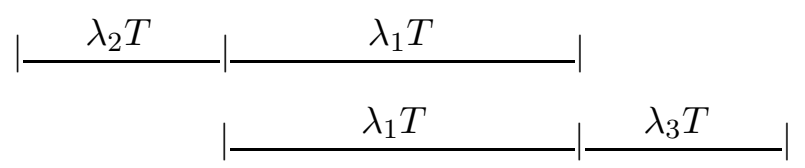

Figure 5: Illustration of Example 3. Example 3 explicitly calculates the extended estimators for data missing in a non-monotonic pattern. The notation above the horizontal lines refers to the length of each segment as a function of the sample size $T$.

We refer to the length of the middle data segment as $\lambda_{1} T$ because all data are observed over this segment. We could let $\lambda_{2} T$ denote the length of the first or the last data segment. Without loss of generality, we let it refer to the length of the first segment. We let $\lambda_{3} T=\left(1-\lambda_{1}-\lambda_{2}\right) T$ denote the length of the final segment. Following the notation convention of Section 2 and the previous examples, we group the moment conditions observed for the first two intervals into a vector $f_{1}\left(x_{1 t}, \theta\right)$ and the moment conditions observed for the last two intervals into a vector $f_{2}\left(x_{2 t}, \theta\right)$. The notation for sub-vectors of $g$ and submatrices of $D_{0}$ and $S$ follows the same convention as in the previous 
example.

The moment conditions for the over-identified estimator $h_{T}^{\mathcal{I}_{3}}$ are

$$
h_{T}^{\mathcal{I}_{3}}(\theta)=\left[g_{1, \lambda_{1} T}(\theta)^{\top} g_{2, \lambda_{1} T}(\theta)^{\top} g_{1, \lambda_{2} T}(\theta)^{\top} g_{2, \lambda_{3} T}(\theta)^{\top}\right]^{\top} .
$$

Then the results in Section 6.1 imply that $\sqrt{T} h_{T}^{\mathcal{I}_{3}}(\theta)$ has asymptotic variance

$$
S^{\mathcal{I}_{3}}=\left[\begin{array}{cccc}
\frac{1}{\lambda_{1}} S_{11} & \frac{1}{\lambda_{1}} S_{12} & 0 & 0 \\
\frac{1}{\lambda_{1}} S_{21} & \frac{1}{\lambda_{1}} S_{22} & 0 & 0 \\
0 & 0 & \frac{1}{\lambda_{2}} S_{11} & 0 \\
0 & 0 & 0 & \frac{1}{\lambda_{3}} S_{22}
\end{array}\right] .
$$

The extended over-identified estimator is therefore

$$
\hat{\theta}_{T}^{\mathcal{I}_{3}}=\operatorname{argmin}_{\theta} h_{T}^{\mathcal{I}_{3}}(\theta)^{\top} W_{T}^{\mathcal{I}_{3}} h_{T}^{\mathcal{I}_{3}}(\theta)
$$

for $W_{T}^{\mathcal{I}_{3}}$ positive definite and $W_{T}^{\mathcal{I}_{3}} \rightarrow$ a.s. $\left(S^{\mathcal{I}_{3}}\right)^{-1}$. The asymptotic distribution is

$$
\sqrt{T}\left(\hat{\theta}_{T}^{\mathcal{I}_{3}}-\theta_{0}\right) \rightarrow_{\mathrm{d}} N\left(0,\left(\left(D_{0}^{\mathcal{I}_{3}}\right)^{\top}\left(S^{\mathcal{I}_{3}}\right)^{-1}\left(D_{0}^{\mathcal{I}_{3}}\right)\right)^{-1}\right)
$$

where

$$
D^{\mathcal{I}_{3}}=\left[\begin{array}{llll}
D_{0,1}^{\top} & D_{0,2}^{\top} & D_{0,1}^{\top} & D_{0,2}^{\top}
\end{array}\right]^{\top} .
$$

We now describe the adjusted-moment estimator. The first two steps in constructing the adjusted-moment estimator are identical to those in the first example. Therefore we can write

$$
h_{\left(\lambda_{1}+\lambda_{2}\right) T}^{\mathcal{A}_{2}}=\left(\begin{array}{l}
g_{1,\left(\lambda_{1}+\lambda_{2}\right) T} \\
g_{2, \lambda_{1} T}+\frac{\lambda_{2}}{\lambda_{1}+\lambda_{2}} B_{21}\left(g_{1, \lambda_{2} T}-g_{1, \lambda_{1} T}\right)
\end{array}\right) .
$$

We have

$$
h_{T}^{\mathcal{A}_{3}}=h_{\left(\lambda_{1}+\lambda_{2}\right) T}^{\mathcal{A}_{2}}-B^{\mathcal{A}_{2}}\left(h_{2,\left(\lambda_{1}+\lambda_{2}\right) T}^{\mathcal{A}_{2}}-g_{2, \lambda_{3} T}\right),
$$

where

$$
\begin{aligned}
B^{\mathcal{A}_{2}}=\lim _{T \rightarrow \infty} E\left[T h_{\left(\lambda_{1}+\lambda_{2}\right) T}^{\mathcal{A}_{2}}\left(h_{2,\left(\lambda_{1}+\lambda_{2}\right) T}^{\mathcal{A}_{2}}-g_{2, \lambda_{3} T}\right)^{\top}\right] & \\
& \times E\left(\left[T\left(h_{2,\left(\lambda_{1}+\lambda_{2}\right) T}^{\mathcal{A}_{2}}-g_{2, \lambda_{3} T}\right)\left(h_{2,\left(\lambda_{1}+\lambda_{2}\right) T}^{\mathcal{A}_{2}}-g_{2, \lambda_{3} T}\right)^{\top}\right]\right)^{-1},
\end{aligned}
$$

where we have suppressed the argument $\theta_{0}$. Define

$$
\gamma=\frac{\lambda_{2}}{\lambda_{1}+\lambda_{2}} \frac{\lambda_{3}}{\lambda_{1}+\lambda_{3}}
$$


Standard arguments (given in the Appendix) show that

$$
B^{\mathcal{A}_{2}}=\frac{\lambda_{3}}{\lambda_{1}+\lambda_{3}}\left(\begin{array}{l}
\frac{\lambda_{1}}{\lambda_{1}+\lambda_{2}} S_{12} \\
S_{22}-\frac{\lambda_{2}}{\lambda_{1}+\lambda_{2}} S_{21} S_{11}^{-1} S_{12}
\end{array}\right) S_{22}^{-1}\left(I-\gamma B_{21} B_{12}\right)^{-1} .
$$

Given $B^{\mathcal{A}_{2}}$, (87) gives the moments for the adjusted-moment estimator. The first component is as follows:

$h_{1 T}^{\mathcal{A}_{3}}=g_{1,\left(\lambda_{1}+\lambda_{2}\right) T}+\frac{\lambda_{1}}{\lambda_{1}+\lambda_{2}} \frac{\lambda_{3}}{\lambda_{1}+\lambda_{3}} B_{12}\left(I-\gamma B_{21} B_{12}\right)^{-1}\left(g_{2, \lambda_{3} T}-g_{2, \lambda_{1} T}-\frac{\lambda_{2}}{\lambda_{1}+\lambda_{2}} B_{21}\left(g_{1, \lambda_{2} T}-g_{1, \lambda_{1} T}\right)\right)$,

while more extensive matrix algebra results in the following expression for the second component:

$h_{2 T}^{\mathcal{A}_{3}}=g_{2,\left(\lambda_{1}+\lambda_{3}\right) T}+\frac{\lambda_{1}}{\lambda_{1}+\lambda_{3}} \frac{\lambda_{2}}{\lambda_{1}+\lambda_{2}}\left(I-\gamma B_{21} B_{12}\right)^{-1} B_{21}\left(g_{1, \lambda_{2} T}-g_{1, \lambda_{1} T}-\frac{\lambda_{3}}{\lambda_{1}+\lambda_{3}} B_{12}\left(g_{2, \lambda_{3} T}-g_{2, \lambda_{1} T}\right)\right)$.

Because

$$
B_{12}\left(I-\gamma B_{21} B_{12}\right)^{-1}=\left(I-\gamma B_{12} B_{21}\right)^{-1} B_{12},
$$

these expressions are symmetric. ${ }^{19}$

At first glance, the adjustments implicit in (89) and (90) do not seem as intuitive as their counterparts in Section 2, or, for that matter, in Sections 6.3.1 and 6.3.2. However, there is a reason for the apparently strange form. It follows from (92) and (78) that (89) can be rewritten as

$$
\begin{aligned}
& h_{1 T}^{\mathcal{A}_{3}}=\frac{\lambda_{2}}{\lambda_{1}+\lambda_{2}} g_{1, \lambda_{2} T}+\frac{\lambda_{1}}{\lambda_{1}+\lambda_{2}} g_{1, \lambda_{1} T}+ \\
& \frac{\lambda_{1}}{\lambda_{1}+\lambda_{2}} \frac{\lambda_{3}}{\lambda_{1}+\lambda_{3}} B_{12}\left(\sum_{m=0}^{\infty}\left(\gamma B_{21} B_{12}\right)^{m}\right)\left(g_{2, \lambda_{3} T}-g_{2, \lambda_{1} T}-\frac{\lambda_{2}}{\lambda_{1}+\lambda_{2}} B_{21}\left(g_{1, \lambda_{2} T}-g_{1, \lambda_{1} T}\right)\right) .
\end{aligned}
$$

It is instructive to expand out the infinite sum explicitly:

$$
\begin{aligned}
h_{1 T}^{\mathcal{A}_{3}}=\frac{\lambda_{2}}{\lambda_{1}+\lambda_{2}} g_{1, \lambda_{2} T}+ & \frac{\lambda_{1}}{\lambda_{1}+\lambda_{2}} g_{1, \lambda_{1} T}+\frac{\lambda_{1}}{\lambda_{1}+\lambda_{2}} \frac{\lambda_{3}}{\lambda_{1}+\lambda_{3}} B_{12}\left(g_{2, \lambda_{3} T}-g_{2, \lambda_{1} T}\right) \\
& -\frac{\lambda_{1}}{\lambda_{1}+\lambda_{2}} \frac{\lambda_{3}}{\lambda_{1}+\lambda_{3}} B_{12} \frac{\lambda_{2}}{\lambda_{1}+\lambda_{2}} B_{21}\left(g_{1, \lambda_{2} T}-g_{1, \lambda_{1} T}\right) \\
& +\frac{\lambda_{1}}{\lambda_{1}+\lambda_{2}} \frac{\lambda_{3}}{\lambda_{1}+\lambda_{3}} B_{12} \frac{\lambda_{2}}{\lambda_{1}+\lambda_{2}} B_{21} \frac{\lambda_{3}}{\lambda_{1}+\lambda_{3}} B_{12}\left(g_{2, \lambda_{3} T}-g_{2, \lambda_{1} T}\right)-\ldots
\end{aligned}
$$

\footnotetext{
${ }^{19}$ Equation (91) can be shown by noting that
}

$$
\left(I-\gamma B_{21} B_{12}\right)^{-1}=\sum_{m=0}^{\infty} \gamma^{m}\left(B_{21} B_{12}\right)^{m} .
$$


The first two terms are partial sums of $g$ over the data segment of length $\lambda_{2} T$ and the data segment of length $\lambda_{1} T$, weighted appropriately. The third term is the adjustment to $g_{1, \lambda_{1} T}$, given that $g_{2}$ is observed over the longer data segment (precisely, the segment of length $\left(\lambda_{1}+\lambda_{3}\right) T$ ). This is the same adjustment as in Section 2, except here it is the first rather than the second series that is being adjusted. Because $g_{1, \lambda_{1} T}$ is weighted by $\lambda_{1} /\left(\lambda_{1}+\lambda_{2}\right)$, the adjustment also receives this weight. Note that there is no adjustment to $g_{1, \lambda_{2} T}$ because the second data series is not observed over the period of length $\lambda_{2} T$.

One possibility would be to stop with the third term. However, the resulting estimator would be inefficient relative to the generalized adjusted-moment estimator. Instead, the extended adjustedmoment estimator has additional terms. The reason is that the adjustment, $\frac{\lambda_{3}}{\lambda_{1}+\lambda_{3}} B_{12}\left(g_{2, \lambda_{3} T}-\right.$ $\left.g_{2, \lambda_{1} T}\right)$, must itself be adjusted to reflect the fact that the first set of series is observed over the data segment of length $\lambda_{2} T$. More precisely, $-\frac{\lambda_{3}}{\lambda_{1}+\lambda_{3}} B_{12} g_{2, \lambda_{1} T}$ must be adjusted. This is the reason for the fourth term. But then this must also be adjusted, and so forth. Repeating this argument results in the telescoping matrix series (94), which, by (92), converges to the extended adjustedmoment estimator (89). A symmetric explanation holds for (90). Thus even in this complicated problem, the adjusted-moment estimator produces moments that have intuitive appeal.

\section{Conclusion}

This paper has introduced two estimators that extend the generalized method of moments of Hansen (1982) to cases where moment conditions are observed over different sample periods. Most estimation procedures, when confronted with data series that are of unequal length, require the researcher to truncate the data so that all series are observed over the same interval. This paper has provided an alternative that allows the researcher to use all the data available for each moment condition.

Under assumptions of mixing and stationarity, we demonstrated consistency, asymptotic normality, and efficiency over standard GMM. Our base case assumed that the two series had the same end date but different start dates. We then generalized our results to cases where the start date and the end date may differ over multiple series. In all cases, using all the data produces more efficient estimates. Interestingly, this gain in efficiency is not only present for the parameters that enter into the moment conditions observed over the longer data. As long as there is some interaction between the moment conditions observed over the long data and the series observed over the short data there is an efficiency gain for all the parameters. This interaction can be through 
covariances between the moment conditions, or through the fact that some parameters appear in both the long-sample and short-sample moment conditions. In an application of our methods to estimation of mutual fund abnormal performance, we show that the effect on standard errors can be substantial.

Our two estimators are as straightforward to implement as standard GMM and have intuitive interpretations. The adjusted-moment estimator calculates moments using all the data available for each series, and then adjusts the moments available over the shorter series using regression coefficients from a regression of the short-series moments on the long-series moments. The overidentified estimator uses the non-overlapping data to form additional moment conditions. These two estimators are equivalent asymptotically, and superior to standard GMM, but differ in finite samples. We leave the question of which estimator has superior finite-sample properties to future work. 


\section{Appendix A}

Lemma A.1 Assume $m \times m$ matrices $U_{1}$ and $U_{2}$ are invertible. If $U_{1}-U_{2}$ is positive semi-definite, then $U_{2}^{-1}-U_{1}^{-1}$ is also positive semi-definite.

Proof See Goldberger (1964, Chapter 2.7).

Lemma A.2 Assume $m \times m$ matrices $U_{1}$ and $U_{2}$ are invertible. If $U_{1}-U_{2}$ is positive semi-definite, then for any conforming matrix $M,\left(M^{\top} U_{1}^{-1} M\right)^{-1}-\left(M^{\top} U_{2}^{-1} M\right)^{-1}$ is also positive semi-definite.

Proof Assume $U_{1}-U_{2}$ is positive semi-definite. By Lemma A.1, $U_{2}^{-1}-U_{1}^{-1}$ is positive semi-definite. For any vector $v$ and matrix $M$,

$$
(M v)^{\top}\left(U_{2}^{-1}-U_{1}^{-1}\right)(M v) \geq 0
$$

Therefore

$$
v^{\top} M^{\top}\left(U_{2}^{-1}-U_{1}^{-1}\right) M v \geq 0
$$

which shows $M^{\top}\left(U_{2}^{-1}-U_{1}^{-1}\right) M$ is positive semi-definite. Applying Lemma A.1 a second time shows that $\left(M^{\top} U_{1}^{-1} M\right)^{-1}-\left(M^{\top} U_{2}^{-1} M\right)^{-1}$ is positive semi-definite as required.

Lemma A.3 Let

$$
S=\left[\begin{array}{ll}
S_{11} & S_{12} \\
S_{21} & S_{22}
\end{array}\right]
$$

be a symmetric invertible matrix. Then

$$
S^{-1}=\left[\begin{array}{cc}
S_{11}^{-1}+B_{21}^{\top} \Sigma^{-1} B_{21} & -B_{21}^{\top} \Sigma^{-1} \\
-\Sigma^{-1} B_{21} & \Sigma^{-1}
\end{array}\right],
$$

where $\Sigma$ is defined by (7). Moreover, if $\bar{S}$ is defined as

$$
\bar{S}=\left[\begin{array}{cc}
\lambda S_{11} & \lambda S_{12} \\
\lambda S_{21} & S_{22}-(1-\lambda) S_{21} S_{11}^{-1} S_{12}
\end{array}\right]
$$

with $\lambda \neq 0$, then

$$
\bar{S}^{-1}=\left[\begin{array}{cc}
\frac{1}{\lambda} S_{11}^{-1}+B_{21}^{\top} \Sigma^{-1} B_{12} & -B_{21}^{\top} \Sigma^{-1} \\
-\Sigma^{-1} B_{12} & \Sigma^{-1}
\end{array}\right]
$$

Proof The first statement follows from the expression for the matrix inverse (see e.g. Green (1997, Chapter 2)). Applying the same formula to $\bar{S}$ results in

$$
\bar{S}^{-1}=\left[\begin{array}{cc}
\bar{S}_{11}^{-1}+\bar{B}_{21}^{\top} \bar{\Sigma}^{-1} \bar{B}_{21} & -\bar{B}_{21}^{\top} \bar{\Sigma}^{-1} \\
-\bar{\Sigma}^{-1} \bar{B}_{12} & \bar{\Sigma}^{-1}
\end{array}\right],
$$


where

$$
\bar{B}_{21}=\bar{S}_{21}\left(\bar{S}_{11}\right)^{-1}=S_{21} S_{11}^{-1}=B_{21}
$$

and

$$
\begin{aligned}
\bar{\Sigma} & =\bar{S}_{22}-\bar{S}_{21} \bar{S}_{11}^{-1} \bar{S}_{12} \\
& =S_{22}-(1-\lambda) S_{21} S_{11}^{-1} S_{12}-\lambda S_{21} S_{11}^{-1} S_{12}=\Sigma
\end{aligned}
$$

Therefore (96) holds.

\section{Proof of Theorem 6.11:}

By Theorem 6.10, it suffices to show that the asymptotic variance of $\sqrt{T} \hat{\theta}_{T}^{\mathcal{I}_{n}}$ is the same as the asymptotic variance of $\sqrt{T} \hat{\theta}_{T}^{\mathcal{A}_{n}}$. The proof is by induction on $n$. For $n=1$,

$$
\theta_{\lambda_{1} T}^{\mathcal{I}_{1}}=\theta_{\lambda_{1} T}^{\mathcal{A}_{1}}
$$

because they both equal the standard GMM estimator over data of length $\lambda_{1} T$. We assume by induction that

$$
D_{0}^{\top}\left(S^{\mathcal{A}_{n-1}}\right)^{-1} D_{0}=\left(D_{0}^{\mathcal{I}_{n-1}}\right)^{\top}\left(S^{\mathcal{I}_{n-1}}\right)^{-1} D_{0}^{\mathcal{I}_{n-1}}
$$

Without loss of generality, let $\phi_{n}=\left\{1, \ldots, \pi_{n}\right\}$. That is, the first $\pi_{n}$ moment conditions are observed over data region $\lambda_{n}$. By (60) it suffices to show

$$
D_{0}^{\top}\left(S^{\mathcal{A}_{n}}\right)^{-1} D_{0}-\left(1-\lambda_{n}\right) D_{0}^{\top}\left(S^{\mathcal{A}_{n-1}}\right)^{-1} D_{0}=\lambda_{n} D_{0, \phi_{n}}^{\top} S_{\phi_{n}}^{-1} D_{0, \phi_{n}}
$$

Equivalently, it suffices to show

$$
\left(S^{\mathcal{A}_{n}}\right)^{-1}=\left(1-\lambda_{n}\right)\left(S^{\mathcal{A}_{n-1}}\right)^{-1}+\lambda_{n}\left[\begin{array}{cc}
S_{\phi_{n}}^{-1} & 0 \\
0 & 0
\end{array}\right]
$$

We use the formula for the inverse of a partitioned matrix (Lemma A.3). Let $-\phi_{n}$ denote the set of data series not observed over $\lambda_{n}$, i.e. the complement of $\phi_{n}$. The assumption that $\phi_{n}=\left\{1, \ldots, \pi_{n}\right\}$ implies that $S^{\mathcal{A}_{n}}$ can be written as

$$
S^{\mathcal{A}_{n}}=\left[\begin{array}{cc}
S_{\phi_{n}}^{\mathcal{A}_{n}} & S_{\phi_{n},-\phi_{n}}^{\mathcal{A}_{n}} \\
S_{-\phi_{n}, \phi_{n}}^{\mathcal{A}_{n}} & S_{-\phi_{n}}^{\mathcal{A}_{n}}
\end{array}\right]
$$

where

$$
S_{-\phi_{n}}^{\mathcal{A}_{n}}=E\left[T h_{-\phi_{n}, T}^{\mathcal{A}_{n}}\left(\theta_{0}\right) h_{-\phi_{n}, T}^{\mathcal{A}_{n}}\left(\theta_{0}\right)^{\top}\right]
$$




$$
S_{-\phi_{n}, \phi_{n}}^{\mathcal{A}_{n}}=E\left[T h_{-\phi_{n}, T}^{\mathcal{A}_{n}}\left(\theta_{0}\right) h_{\phi_{n}, T}^{\mathcal{A}_{n}}\left(\theta_{0}\right)^{\top}\right]
$$

and $S_{\phi_{n},-\phi_{n}}^{\mathcal{A}_{n}}=\left(S_{-\phi_{n}, \phi_{n}}^{\mathcal{A}_{n}}\right)^{\top}$. Note that under this ordering,

$$
B^{\mathcal{A}_{n-1}}=\frac{1}{1-\lambda_{n}}\left(\begin{array}{c}
S_{\phi_{n}}^{\mathcal{A}_{n-1}} \\
S_{-\phi_{n}, \phi_{n}}^{\mathcal{A}_{n-1}}
\end{array}\right)\left[\frac{1}{\lambda_{n}} S_{\phi_{n}}+\frac{1}{1-\lambda_{n}} S_{\phi_{n}}^{\mathcal{A}_{n-1}}\right]^{-1} .
$$

Analogously to $B_{21}$ in Section 2, define

$$
\begin{aligned}
B_{21}^{\mathcal{A}_{n}} & =S_{-\phi_{n}, \phi_{n}}^{\mathcal{A}_{n}}\left(S_{\phi_{n}}^{\mathcal{A}_{n}}\right)^{-1} \\
B_{21}^{\mathcal{A}_{n-1}} & =S_{-\phi_{n}, \phi_{n}}^{\mathcal{A}_{n-1}}\left(S_{\phi_{n}}^{\mathcal{A}_{n-1}}\right)^{-1} .
\end{aligned}
$$

Analogously to $\Sigma$ in Section 2, define

$$
\begin{aligned}
\Sigma^{\mathcal{A}_{n}} & =S_{-\phi_{n},-\phi_{n}}^{\mathcal{A}_{n}}-S_{-\phi_{n}, \phi_{n}}^{\mathcal{A}_{n}}\left(S_{\phi_{n}}^{\mathcal{A}_{n}}\right)^{-1} S_{\phi_{n},-\phi_{n}}^{\mathcal{A}_{n}} \\
\Sigma^{\mathcal{A}_{n-1}} & =S_{-\phi_{n},-\phi_{n}}^{\mathcal{A}_{n-1}}-S_{-\phi_{n}, \phi_{n}}^{\mathcal{A}_{n-1}}\left(S_{\phi_{n}}^{\mathcal{A}_{n-1}}\right)^{-1} S_{\phi_{n},-\phi_{n}}^{\mathcal{A}_{n-1}} .
\end{aligned}
$$

By Lemma A.3, (97) holds if and only if

$$
\begin{aligned}
\left(S_{\phi_{n}}^{\mathcal{A}_{n}}\right)^{-1} & =\left(1-\lambda_{n}\right)\left(S_{\phi_{n}}^{\mathcal{A}_{n-1}}\right)^{-1}+\lambda_{n}\left(S_{\phi_{n}}\right)^{-1} \\
B_{21}^{\mathcal{A}_{n}} & =B_{21}^{\mathcal{A}_{n-1}} \\
\Sigma^{\mathcal{A}_{n}} & =\frac{1}{1-\lambda_{n}} \Sigma^{\mathcal{A}_{n-1}} .
\end{aligned}
$$

We first show (103). Equation (69) implies

$$
S_{\phi_{n}}^{\mathcal{A}_{n}}=\frac{1}{1-\lambda_{n}} S_{\phi_{n}}^{\mathcal{A}_{n-1}}-\frac{1}{1-\lambda_{n}} S_{\phi_{n}}^{\mathcal{A}_{n-1}}\left[\frac{1}{\lambda_{n}} S_{\phi_{n}}+\frac{1}{1-\lambda_{n}} S_{\phi_{n}}^{\mathcal{A}_{n-1}}\right]^{-1} \frac{1}{1-\lambda_{n}} S_{\phi_{n}}^{\mathcal{A}_{n-1}} .
$$

Pre-multiplying by $\left(1-\lambda_{n}\right)\left(S_{\phi_{n}}^{\mathcal{A}_{n-1}}\right)^{-1}$ yields

$$
\begin{aligned}
\left(1-\lambda_{n}\right)\left(S_{\phi_{n}}^{\mathcal{A}_{n-1}}\right)^{-1} S_{\phi_{n}}^{\mathcal{A}_{n}} & =I-\left[\frac{1}{\lambda_{n}} S_{\phi_{n}}+\frac{1}{1-\lambda_{n}} S_{\phi_{n}}^{\mathcal{A}_{n-1}}\right]^{-1} \frac{1}{1-\lambda_{n}} S_{\phi_{n}}^{\mathcal{A}_{n-1}} \\
& =\left[\frac{1}{\lambda_{n}} S_{\phi_{n}}+\frac{1}{1-\lambda_{n}} S_{\phi_{n}}^{\mathcal{A}_{n-1}}\right]^{-1} \frac{1}{\lambda_{n}} S_{\phi_{n}} .
\end{aligned}
$$

Taking inverses yields

$$
\begin{aligned}
\frac{1}{1-\lambda_{n}}\left(S_{\phi_{n}}^{\mathcal{A}_{n}}\right)^{-1} S_{\phi_{n}}^{\mathcal{A}_{n-1}} & =\lambda_{n}\left(S_{\phi_{n}}\right)^{-1}\left[\frac{1}{\lambda_{n}} S_{\phi_{n}}+\frac{1}{1-\lambda_{n}} S_{\phi_{n}}^{\mathcal{A}_{n-1}}\right] \\
& =I+\frac{\lambda_{n}}{1-\lambda_{n}}\left(S_{\phi_{n}}\right)^{-1} S_{\phi_{n}}^{\mathcal{A}_{n-1}} .
\end{aligned}
$$


Post-multiplying by $\left(1-\lambda_{n}\right)\left(S_{\phi_{n}}^{\mathcal{A}_{n-1}}\right)^{-1}$ yields $(103)$.

We now show (104). By (69),

$$
S_{-\phi_{n}, \phi_{n}}^{\mathcal{A}_{n}}=\frac{1}{1-\lambda_{n}} S_{-\phi_{n}, \phi_{n}}^{\mathcal{A}_{n-1}}-\frac{1}{1-\lambda_{n}} S_{-\phi_{n}, \phi_{n}}^{\mathcal{A}_{n-1}}\left[\frac{1}{\lambda_{n}} S_{\phi_{n}}+\frac{1}{1-\lambda_{n}} S_{\phi_{n}}^{\mathcal{A}_{n-1}}\right]^{-1} \frac{1}{1-\lambda_{n}} S_{\phi_{n}}^{\mathcal{A}_{n-1}} .
$$

Post-multiplying by $\left(S_{\phi_{n}}^{\mathcal{A}_{n}}\right)^{-1}$ and applying (103) produces

$$
\begin{aligned}
B_{21}^{\mathcal{A}_{n}} & =S_{-\phi_{n}, \phi_{n}}^{\mathcal{A}_{n}}\left(S_{\phi_{n}}^{\mathcal{A}_{n}}\right)^{-1}=\frac{1}{1-\lambda_{n}} S_{-\phi_{n}, \phi_{n}}^{\mathcal{A}_{n-1}}\left[\left(1-\lambda_{n}\right)\left(S_{\phi_{n}}^{\mathcal{A}_{n-1}}\right)^{-1}+\lambda_{n} S_{\phi_{n}}^{-1}\right] \\
& -\frac{1}{1-\lambda_{n}} S_{-\phi_{n}, \phi_{n}}^{\mathcal{A}_{n-1}}\left[\frac{1}{\lambda_{n}} S_{\phi_{n}}+\frac{1}{1-\lambda_{n}} S_{\phi_{n}}^{\mathcal{A}_{n-1}}\right]^{-1} \frac{1}{1-\lambda_{n}} S_{\phi_{n}}^{\mathcal{A}_{n-1}}\left[\left(1-\lambda_{n}\right)\left(S_{\phi_{n}}^{\mathcal{A}_{n-1}}\right)^{-1}+\lambda_{n} S_{\phi_{n}}^{-1}\right] .
\end{aligned}
$$

Expanding out the first term on the right hand side and multiplying through by $S_{\phi_{n}}^{\mathcal{A}_{n-1}}$ in the second term produces

$$
\begin{aligned}
B_{21}^{\mathcal{A}_{n}}=B_{21}^{\mathcal{A}_{n-1}}+\frac{\lambda_{n}}{1-\lambda_{n}} S_{-\phi_{n}, \phi_{n}}^{\mathcal{A}_{n-1}} S_{\phi_{n}}^{-1} & \\
& \quad-\frac{1}{1-\lambda_{n}} S_{-\phi_{n}, \phi_{n}}^{\mathcal{A}_{n-1}}\left[\frac{1}{\lambda_{n}} S_{\phi_{n}}+\frac{1}{1-\lambda_{n}} S_{\phi_{n}}^{\mathcal{A}_{n-1}}\right]^{-1}\left[I+\frac{\lambda_{n}}{1-\lambda_{n}} S_{\phi_{n}}^{\mathcal{A}_{n-1}} S_{\phi_{n}}^{-1}\right] .
\end{aligned}
$$

Factoring out $\lambda_{n} S_{\phi_{n}}^{-1}$ in the last term yields (104).

Lastly, we show (105). Equation (69) implies

$$
S_{-\phi_{n},-\phi_{n}}^{\mathcal{A}_{n}}=\frac{1}{1-\lambda_{n}} S_{-\phi_{n},-\phi_{n}}^{\mathcal{A}_{n-1}}-\frac{1}{1-\lambda_{n}} S_{-\phi_{n}, \phi_{n}}^{\mathcal{A}_{n-1}}\left[\frac{1}{\lambda_{n}} S_{\phi_{n}}+\frac{1}{1-\lambda_{n}} S_{\phi_{n}}^{\mathcal{A}_{n-1}}\right]^{-1} \frac{1}{1-\lambda_{n}} S_{\phi_{n},-\phi_{n}}^{\mathcal{A}_{n-1}} .
$$

By (104),

$$
\begin{aligned}
S_{-\phi_{n}, \phi_{n}}^{\mathcal{A}_{n}}\left(S_{\phi_{n}}^{\mathcal{A}_{n}}\right)^{-1} S_{\phi_{n},-\phi_{n}}^{\mathcal{A}_{n}} & =B_{21}^{\mathcal{A}_{n}} S_{\phi_{n}}^{\mathcal{A}_{n}}\left(B_{21}^{\mathcal{A}_{n}}\right)^{\top} \\
& =B_{21}^{\mathcal{A}_{n-1}} S_{\phi_{n}}^{\mathcal{A}_{n}}\left(B_{21}^{\mathcal{A}_{n-1}}\right)^{\top} .
\end{aligned}
$$

Therefore,

$$
\begin{aligned}
S_{-\phi_{n}, \phi_{n}}^{\mathcal{A}_{n}}\left(S_{\phi_{n}}^{\mathcal{A}_{n}}\right)^{-1} S_{\phi_{n},-\phi_{n}}^{\mathcal{A}_{n}} & =S_{-\phi_{n}, \phi_{n}}^{\mathcal{A}_{n-1}}\left(S_{\phi_{n}}^{\mathcal{A}_{n-1}}\right)^{-1}\left[\left(1-\lambda_{n}\right)\left(S_{\phi_{n}}^{\mathcal{A}_{n-1}}\right)^{-1}+\lambda_{n} S_{\phi_{n}}^{-1}\right]^{-1}\left(S_{\phi_{n}}^{\mathcal{A}_{n-1}}\right)^{-1} S_{\phi_{n},-\phi_{n}}^{\mathcal{A}_{n-1}} \\
& =S_{-\phi_{n}, \phi_{n}}^{\mathcal{A}_{n-1}}\left[\left(1-\lambda_{n}\right) S_{\phi_{n}}+\lambda_{n} S_{\phi_{n}}^{\mathcal{A}_{n-1}}\right]^{-1} S_{\phi_{n}}\left(S_{\phi_{n}}^{\mathcal{A}_{n-1}}\right)^{-1} S_{\phi_{n},-\phi_{n}}^{\mathcal{A}_{n-1}} .
\end{aligned}
$$

Substituting in (106) and (107) into (101),

$$
\begin{aligned}
\Sigma^{\mathcal{A}_{n}}= & \frac{1}{1-\lambda_{n}} S_{-\phi_{n},-\phi_{n}}^{\mathcal{A}_{n-1}}- \\
& \quad \frac{1}{1-\lambda_{n}} S_{-\phi_{n}, \phi_{n}}^{\mathcal{A}_{n-1}}\left[\frac{1}{\lambda_{n}} S_{\phi_{n}}+\frac{1}{1-\lambda_{n}} S_{\phi_{n}}^{\mathcal{A}_{n-1}}\right]^{-1}\left[\frac{1}{1-\lambda_{n}} I+\frac{1}{\lambda_{n}} S_{\phi_{n}}\left(S_{\phi_{n}}^{\mathcal{A}_{n-1}}\right)^{-1}\right] S_{\phi_{n},-\phi_{n}}^{\mathcal{A}_{n-1}} .
\end{aligned}
$$


Which implies

$$
\begin{aligned}
\Sigma^{\mathcal{A}_{n}}= & \frac{1}{1-\lambda_{n}} S_{-\phi_{n},-\phi_{n}}^{\mathcal{A}_{n-1}}- \\
& \frac{1}{1-\lambda_{n}} S_{-\phi_{n}, \phi_{n}}^{\mathcal{A}_{n-1}}\left[\frac{1}{\lambda_{n}} S_{\phi_{n}}+\frac{1}{1-\lambda_{n}} S_{\phi_{n}}^{\mathcal{A}_{n-1}}\right]^{-1}\left[\frac{1}{1-\lambda_{n}} S_{\phi_{n}}^{\mathcal{A}_{n-1}}+\frac{1}{\lambda_{n}} S_{\phi_{n}}\right]\left(S_{\phi_{n}}^{\mathcal{A}_{n-1}}\right)^{-1} S_{\phi_{n},-\phi_{n}}^{\mathcal{A}_{n-1}} \\
= & \frac{1}{1-\lambda_{n}} S_{-\phi_{n},-\phi_{n}}^{\mathcal{A}_{n-1}}-\frac{1}{1-\lambda_{n}} S_{-\phi_{n}, \phi_{n}}^{\mathcal{A}_{n-1}}\left(S_{\phi_{n}}^{\mathcal{A}_{n-1}}\right)^{-1} S_{\phi_{n},-\phi_{n}}^{\mathcal{A}_{n-1}} \\
= & \frac{1}{1-\lambda_{n}} \Sigma^{\mathcal{A}_{n-1}}
\end{aligned}
$$

which shows (105).

Proof that $B^{\mathcal{A}_{2}}$ in Section 6.3.3 is equal to (88):

From Theorem 6.1 and (86), it follows that

$$
\begin{aligned}
\lim _{T \rightarrow \infty} E & {\left[T\left(h_{2,\left(\lambda_{1}+\lambda_{2}\right) T}^{\mathcal{A}_{2}}-g_{2, \lambda_{3} T}\right)\left(h_{2,\left(\lambda_{1}+\lambda_{2}\right) T}^{\mathcal{A}_{2}}-g_{2, \lambda_{3} T}\right)^{\top}\right] } \\
& =\left(\frac{1}{\lambda_{3}}+\frac{1}{\lambda_{1}}\right) S_{22}+\left(\frac{\lambda_{2}}{\lambda_{1}+\lambda_{2}}\right)^{2}\left(\frac{1}{\lambda_{2}}+\frac{1}{\lambda_{1}}\right) B_{21} S_{11} B_{21}^{\top}-\frac{1}{\lambda_{1}} \frac{\lambda_{2}}{\lambda_{1}+\lambda_{2}}\left(S_{21} B_{21}^{\top}+B_{21} S_{12}\right) \\
& =\left(\frac{1}{\lambda_{3}}+\frac{1}{\lambda_{1}}\right) S_{22}-\frac{1}{\lambda_{1}} \frac{\lambda_{2}}{\lambda_{1}+\lambda_{2}} S_{21} S_{11}^{-1} S_{12}
\end{aligned}
$$

and

$$
\begin{aligned}
\lim _{T \rightarrow \infty} E\left[T h_{1,\left(\lambda_{1}+\lambda_{2}\right) T}^{\mathcal{A}_{2}}\left(h_{2,\left(\lambda_{1}+\lambda_{2}\right) T}^{\mathcal{A}_{2}}-g_{2, \lambda_{3} T}\right)^{\top}\right] & =\lim _{T \rightarrow \infty} E\left[T g_{1,\left(\lambda_{1}+\lambda_{2}\right) T}\left(h_{2,\left(\lambda_{1}+\lambda_{2}\right) T}^{\mathcal{A}_{2}}\right)^{\top}\right] \\
& =\frac{1}{\lambda_{1}+\lambda_{2}} S_{12} .
\end{aligned}
$$

where we have applied the reasoning of (21). (109) multiplied by the inverse of (108) equals the first component of $B^{\mathcal{A}_{2}}$. The second component equals

$$
\begin{aligned}
\lim _{T \rightarrow \infty} E\left[T h_{2,\left(\lambda_{1}+\lambda_{2}\right) T}^{\mathcal{A}_{2}}\left(h_{2,\left(\lambda_{1}+\lambda_{2}\right) T}^{\mathcal{A}_{2}}-g_{2, \lambda_{3} T}\right)^{\top}\right] & =\lim _{T \rightarrow \infty} E\left[T h_{2,\left(\lambda_{1}+\lambda_{2}\right)}^{\mathcal{A}_{2}}\left(h_{2,\left(\lambda_{1}+\lambda_{2}\right) T}^{\mathcal{A}_{2}}\right)^{\top}\right] \\
& =\frac{1}{\lambda_{1}}\left(S_{22}-\frac{\lambda_{2}}{\lambda_{1}+\lambda_{2}} S_{21} S_{11}^{-1} S_{12}\right)
\end{aligned}
$$

multiplied by the inverse of (108). The resulting expression for $B^{\mathcal{A}_{2}}$ can be simplified considerably. The inverse of (108) equals

$$
\begin{aligned}
\left(\left(\frac{1}{\lambda_{3}}+\frac{1}{\lambda_{1}}\right) S_{22}-\frac{1}{\lambda_{1}} \frac{\lambda_{2}}{\lambda_{1}+\lambda_{2}} S_{21} S_{11}^{-1} S_{12}\right)^{-1} & =S_{22}^{-1}\left(I-\gamma S_{21} S_{11}^{-1} S_{12} S_{22}^{-1}\right)^{-1} \frac{\lambda_{1} \lambda_{3}}{\lambda_{1}+\lambda_{3}} \\
& =S_{22}^{-1}\left(I-\gamma B_{21} B_{12}\right)^{-1} \frac{\lambda_{1} \lambda_{3}}{\lambda_{1}+\lambda_{3}}
\end{aligned}
$$


where $B_{12}=S_{12} S_{22}^{-1}$. Therefore,

$$
B^{\mathcal{A}_{2}}=\frac{\lambda_{3}}{\lambda_{1}+\lambda_{3}}\left(\begin{array}{l}
\frac{\lambda_{1}}{\lambda_{1}+\lambda_{2}} S_{12} \\
S_{22}-\frac{\lambda_{2}}{\lambda_{1}+\lambda_{2}} S_{21} S_{11}^{-1} S_{12}
\end{array}\right) S_{22}^{-1}\left(I-\gamma B_{21} B_{12}\right)^{-1}
$$




\section{References}

Amemiya, Takeshi, 1985, Advanced Econometrics. (Harvard University Press Cambridge, MA).

Brandt, Michael W., 1999, Estimating portfolio and consumption choice: A conditional Euler equations approach, Journal of Finance 54, 1609-1645.

Burguete, Jose F., A. Ronald Gallant, and Geraldo Souza, 1982, On unification of the asymptotic theory of nonlinear econometric models, Econometric Reviews 1, 151-190.

Campbell, John Y., and Robert J. Shiller, 1988, The dividend-price ratio and expectations of future dividends and discount factors, Review of Financial Studies 58, 495-514.

Carhart, Mark M., 1997, On persistence in mutual fund performance, Journal of Finance 52, 57-82.

Cochrane, John H., 2001, Asset Pricing. (Princeton University Press Princeton, NJ).

Duffie, Darrell, and Kenneth J. Singleton, 1993, Simulated moments estimation of Markov models of asset prices, Econometrica 61, 929-952.

Elton, Edwin J., Martin J. Gruber, and Christopher R. Blake, 1996, Survivorship bias and mutual fund performance, Review of Financial Studies 9, 1097-1120.

Fama, Eugene F., and Kenneth R. French, 1989, Business conditions and expected returns on stocks and bonds, Journal of Financial Economics 29, 23-49.

Fama, Eugene F., and Kenneth R. French, 1993, Common risk factors in the returns on bonds and stocks, Journal of Financial Economics 33, 3-53.

Ferson, Wayne E., and Rudi W. Schadt, 1996, Measuring fund strategy and performance in changing economic conditions, Journal of Finance 51, 425-461.

Goldberger, Arthur S., 1964, Econometric Theory. (John Wiley and Sons New York).

Green, William H., 1997, Econometric Analysis. (Prentice-Hall, Inc. Upper Saddle River, NJ).

Hansen, Lars Peter, 1982, Large sample properties of generalized method of moments estimators, Econometrica 50, 1029-1054.

Hansen, Lars Peter, and Ken Singleton, 1982, Generalized instrumental variables estimation of nonlinear rational expectations models, Econometrica 50, 1269-1286. 
Kandel, Shmuel, and Robert F. Stambaugh, 1996, On the predictability of stock returns: An asset allocation perspective, Journal of Finance 51, 385-424.

Lettau, Martin, and Sydney C. Ludvigson, 2001, Resurrecting the (C)CAPM: A Cross-Sectional Test When Risk Premia are Time-Varying, Journal of Political Economy 109, 1238-1287.

Little, Roderick J. A., and Donald B. Rubin, 2002, Statistical analysis with missing data. (John Wiley \& Sons Hoboken, NJ) 2 edn.

Lynch, Anthony W., Jessica A. Wachter, and Walter Boudry, 2004, Does mutual fund performance vary over the business cycle?, Working Paper, New York University and the University of Pennsylvania.

Newey, Whitnew, and Kenneth West, 1987, A simple, positive-definite, heteroskedastic and autocorrelation consistent covariance matrix, Econometrica 55, 703-708.

Newey, Whitney K., and Daniel McFadden, 1994, Large sample estimation and hypothesis testing, in R.F. Engle, and D. L. McFadden, eds.: Handbook of Econometrics, Volume IV (North-Holland, Amsterdam ).

Pastor, Lubos, and Robert F. Stambaugh, 2002a, Investing in equity mutual funds, Journal of Financial Economics 63, 351-380.

Pastor, Lubos, and Robert F. Stambaugh, 2002b, Mutual fund performance and seemingly unrelated assets, Journal of Financial Economics 63, 315-349.

Robins, James M., and Andrea Rotnitsky, 1995, Semiparametric efficiency in multivariate regression models with missing data, Journal of the American Statistical Association 90, 122-129.

Shanken, Jay, 1990, Intertemporal asset pricing: An empirical investigation, Journal of Econometrics $45,99-120$.

Singleton, Kenneth, 2004, Dynamic asset pricing models: Econometric specifications and empirical assessments. (In preparation).

Stambaugh, Robert, 1997, Analyzing investments whose histories differ in length, Journal of Financial Economics 45, 285-331. 
Storesletten, Kjetil, Chris I. Telmer, and Amir Yaron, 2004, Cyclical dynamics in idiosyncratic labor market risk, Journal of Political Economy 112, 695-717.

White, Halbert, 1994, Asymptotic Theory for Econometricians. (Academic Press, Inc.).

White, Halbert, and Ian Domowitz, 1984, Nonlinear regression with dependent observations, Econometrica 52, 143-162.

Wiesenberger Financial Service, 1977-1992, Investment Companies. (Warren, Gorham \& Lamont, Inc. Boston). 